\title{
GR chaperone cycle mechanism revealed by cryo- EM: the GR-loading complex
}

\section{David Agard ( $\nabla$ agard@msg.ucsf.edu )}

University of California, San Francisco https://orcid.org/0000-0003-3512-695X

\section{Ray Wang}

University of California, San Francisco

\section{Chari Noddings}

University of California, San Francisco https://orcid.org/0000-0003-0890-9035

\section{Elaine Kirschke}

University of California, San Francisco

\section{Alexander Myasnikov}

University of California San Francisco https://orcid.org/0000-0003-2607-7121

Jill Johnson

University of Idaho https://orcid.org/0000-0003-3881-722X

\section{Biological Sciences - Article}

Keywords: GR-loading complex, glucocorticoid receptor, Hsp90, Hsp70

Posted Date: December 28th, 2020

DOI: https://doi.org/10.21203/rs.3.rs-120723/v1

License: (c) (i) This work is licensed under a Creative Commons Attribution 4.0 International License. Read Full License

Version of Record: A version of this preprint was published at Nature on December 22nd, 2021. See the published version at https://doi.org/10.1038/s41586-021-04252-1. 
1 GR chaperone cycle mechanism revealed by cryo-EM: the GR-loading 2 complex

3

4 Ray Yu-Ruei Wang ${ }^{1}$, Chari M. Noddings ${ }^{1}$, Elaine Kirschke ${ }^{1}$, Alexander G. Myasnikov ${ }^{1, \pi}$, 5 Jill L. Johnson ${ }^{2}$, and David A. Agard ${ }^{1 \ddagger}$

6

$7 \quad{ }^{1}$ Department of Biochemistry and Biophysics, University of California San Francisco,

8 San Francisco, CA 94143, USA

9 'Department of Biological Sciences, University of Idaho, Moscow, ID 83844, USA

12 TCurrent address: Department of Structural Biology, St Jude Children's Research 13 Hospital, Memphis, TN 38105, USA

$15 \mp$ †Correspondence to: agard@msg.ucsf.edu 


\section{Abstract}

19 Maintaining a healthy proteome is fundamental for organism survival ${ }^{1,2}$. Integral to this

20 are Hsp90 and Hsp70 molecular chaperones that together facilitate the folding,

21 remodeling and maturation of Hsp90's many "client" proteins ${ }^{3-7}$. The glucocorticoid

22 receptor (GR) is a model client strictly dependent upon Hsp90/Hsp70 for activity ${ }^{8-13}$.

23 Chaperoning GR involves a cycle of inactivation by Hsp70, formation of an inactive

24 GR:Hsp90:Hsp70:Hop "loading" complex, conversion to an active GR:Hsp90:p23

25 "maturation" complex, and subsequent GR release ${ }^{14}$. Unfortunately, a molecular

26 understanding of this intricate chaperone cycle is lacking for any client. Here, we report

27 the cryo-EM structure of the GR loading complex, in which Hsp70 loads GR onto

28 Hsp90, revealing the molecular basis of direct Hsp90/Hsp70 coordination. The structure

29 reveals two Hsp70s - one delivering GR and the other scaffolding Hop. Unexpectedly,

30 the Hop cochaperone interacts with all components of the complex including GR,

31 poising Hsp90 for subsequent ATP hydrolysis. GR is partially unfolded and recognized

32 via an extended binding pocket composed of Hsp90, Hsp70 and Hop, revealing the

33 mechanism of GR loading and inactivation. Together with the GR maturation complex

34 (Noddings et al., 2020), we present the first complete molecular mechanism of

35 chaperone-dependent client remodeling, establishing general principles of client

36 recognition, inhibition, transfer and activation. 


\section{Main}

39 The highly abundant and conserved Hsp90 and Hsp70 molecular chaperones are

40 essential for proteome maintenance. Hsp70 recognizes virtually all unfolded/misfolded

41 proteins, and generally functions early in protein folding ${ }^{15}$. By contrast, Hsp90 typically

42 functions later ${ }^{11}$, targeting a select set of "clients" ${ }^{\prime 7,16}$. Despite the differences, Hsp90

43 and Hsp70 share clients that are highly enriched for signaling and regulatory

44 proteins $\mathrm{s}^{4,16,17}$, making both chaperones important pharmaceutical targets for cancer ${ }^{18,19}$

45 and neurodegenerative diseases ${ }^{20-22}$. Both chaperones are dynamic molecular

46 machines with complex ATP-dependent conformational cycles that drive client

47 binding/release. Hsp70 uses its $\mathrm{N}$-terminal nucleotide-binding domain ( $\mathrm{Hsp} 70_{\mathrm{NBD}}$ ) to

48 allosterically regulate its C-terminal substrate-binding domain ( $\left.\mathrm{Hsp} 70_{\mathrm{SBD}}\right)$, comprising a

$49 \beta$-sandwich core $\left(H_{s p 70}\right.$ sBD- $\left.\beta\right)$ and an $\alpha$-helical lid $\left(H_{s p 70}{ }_{\text {SBD- } \alpha}\right)^{15,23-26}$. In the weak client

50 binding ATP-bound "open" state (Hsp70 ATP), both the Hsp70 sBD- $\alpha$ and Hsp70 $_{\text {SBD- } \beta}$ dock

51 onto the Hsp70 ${ }_{\mathrm{NBD}}{ }^{27}$. In the ADP state (Hsp70ADP), the Hsp70 ${ }_{\mathrm{NBD}}$ and Hsp70 $\mathrm{SBD}$

52 subdomains separate, resulting in a high-affinity client-binding state ${ }^{28}$. Hsp90

53 constitutively dimerizes through its C-terminal domain (Hsp90 сто) and cycles through

54 open and closed conformations acting as a molecular clamp $p^{5,29}$. In the nucleotide-free

55 state $\left(\mathrm{Hsp90}{ }^{\mathrm{Apo}}\right.$ ), Hsp90 populates a variety of open conformations ${ }^{29-31}$, whereas ATP

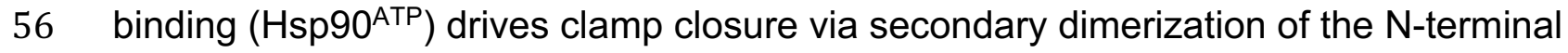

57 domains (Hsp90 $\left.{ }_{\text {NTD }}\right)^{32}$. Clamp closure activates Hsp90 for ATP hydrolysis and is a rate-

58 limiting ${ }^{33}$ process requiring $\mathrm{Hsp} 90_{\mathrm{NTD}}$ rotation, $\mathrm{N}$-terminal helix rotation and ATP-binding

59 pocket lid closure. Unlike Hsp70, Hsp90 can engage clients independent of nucleotide

60 state via the middle domain ${ }^{34,35}$ ( $\left.\mathrm{Hsp} 0_{\mathrm{MD}}\right)$ and the amphipathic helix-hairpins ${ }^{36}$

61 (Hsp90 amphi-a) on the Hsp90 ${ }_{\text {ctD. }}$.

62 The glucocorticoid receptor $(\mathrm{GR})$ is a steroid hormone-activated transcription factor that

63 constitutively depends on Hsp90 to function ${ }^{10}$. Building on the pioneering work of Pratt

64 and Toft ${ }^{8,12,13}$, we previously reconstituted GR's Hsp90 dependence using an in vitro

65 system ${ }^{14}$, establishing a 4-step cycle (Fig. 1a) starting with active GR ligand-binding

66 domain (hereafter GR for simplicity). Next, Hsp70 inactivates GR ligand binding, then

67 co-chaperone Hop (Hsp90/Hsp70 organization protein) helps load Hsp70:GR onto 
Hsp90 forming the inactive "loading" complex (GR:Hsp90:Hsp70:Hop). Upon Hsp90 closure and ATP hydrolysis, Hsp70 and Hop are released, followed by the incorporation of p23, forming the GR:Hsp90:p23 "maturation" complex. In the maturation complex,

$71 \mathrm{GR}$ is reactivated, indicating $\mathrm{GR}$ is conformationally remodeled during the transition. A

72 similar pattern of Hsp70/Hsp90 functional antagonism has subsequently been shown for

73 other clients ${ }^{37-40}$, supporting a general mechanism.

74 Unfortunately, the molecular basis for almost all of this complex chaperone interplay 75 remains unknown, with high-resolution structural studies hampered by the instability of 76 clients and the highly dynamic nature of client:chaperone associations. Here, we report

77 a high-resolution cryo-EM structure of the client-loading complex, providing much needed molecular insights into how Hsp90/Hsp70 coordinate their ATP cycles, how they are organized by Hop, and the molecular mechanisms underlying GR's functional

80 regulation by Hsp90/Hsp70.

\section{Results}

\section{Structure determination and architecture of the client-loading complex}

84 The client-loading complex was prepared by reconstitution using excess ADP to 85 enhance client binding by Hsp70 and an ATP-binding deficient Hsp90 (Hsp90 $\left.{ }^{\mathrm{D} 93 \mathrm{~N}}\right)^{41}$ to

86 stall the cycle at this intermediate step, followed by glutaraldehyde stabilization

87 (Extended Data Fig. 1). A $\sim 3.6 \AA$ resolution cryo-EM reconstruction was obtained from $88 \sim 4$ million particles using RELION ${ }^{42}$ (Extended Data Fig. 2-4, Materials and Methods).

89 The resulting structure reveals an architecture drastically different than expected, with 90 the Hsp90 dimer (Hsp90A/B) surrounded by Hop, GR and unexpectedly two Hsp70s 91 (Hsp70 "C" for client-loading and Hsp70"S" for scaffolding) (Fig. 1b,c,e,f, Extended Data 92 Fig. 5). Hsp90 adopts a previously unseen "semi-closed" conformation, in which the 93 Hsp90 NTDS have rotated into an Hsp90 ATP-like orientation but have not yet reached the

94 fully-closed ATP state (Extended Data Fig. 6). The observed Hsp90NTD orientation is 
95 stabilized by two Hsp70 $0_{\mathrm{NBD}}$ s that bind symmetrically to the Hsp90 $0_{\mathrm{NTD}} / \mathrm{Hsp} 90_{\mathrm{MD}}$ interface

96 of each Hsp90 protomer. Hop intimately interacts with each Hsp90 protomer, the two

97 Hsp70s, and remarkably, a portion of GR. Although the two Hsp70sBDS are not visible in

98 the high-resolution map, the Hsp70 $\mathrm{C}_{\text {SBD- } \beta}$ subdomain becomes visible in low-pass

99 filtered maps (Fig. 1b, Extended Data Fig. 25d). Also seen in the filtered maps, GR is

100 positioned on one side of the Hsp90 dimer (Fig. 1c and Extended Data Fig. 24b,c).

101 Additionally, a lower-resolution map $(\sim 7 \AA)$ was reconstructed showing a loading

102 complex that has lost Hsp70C but retains Hsp70S, Hop and GR (Extended Data Fig. 7).

103 The observation of the two-Hsp70 and one-Hsp70 loading complexes populated in our

104 sample is consistent with a previous study ${ }^{43}$.

105

\section{The nucleotide-regulated interplay between Hsp90 and Hsp70}

107 Two major interfaces are formed in both of the nearly identical $\mathrm{Hsp} 70_{\mathrm{NBD}} / \mathrm{Hsp} 90$

108 protomer interactions (RMSD of $0.96 \AA$, Extended Data Fig. 8a-c). In Interface I, the

109 outer edge of the Hsp90 $0_{M D} \beta$-sheet inserts into the cleft formed by the Hsp70 $0_{\text {NBD-IA }}$ and

$110 \mathrm{Hsp70}_{\text {NBD-IIA }}$ subdomains (Fig. 2a, Extended Data Fig. 9a). Notably, in Hsp70ATP this

111 cleft binds the Hsp70 interdomain linker ${ }^{24,27}$ and also contributes to binding Hsp40's J-

112 domain ${ }^{44}$ (Extended Data Fig. 9b). Hence, the cleft is only available in Hsp70ADP.

113 Interface I is tightly packed ( $479 \AA^{2}$ of buried surface area, BSA), and is stabilized by

114 numerous polar interactions (Fig. 2c,d). This explains the reduced Hsp70 interaction

115 and the growth defects and significant loss of GR and v-Src function caused by

116 mutations of the central residue in this interface Hsp90 3333 (G313 in $\mathrm{yHsp82/G309} \mathrm{in} \mathrm{yHsc82)} \mathrm{(Fig.}$

$1172 \mathrm{2d}$ ) and the 2.5-5 fold reduction in Hsp90/Hsp70 affinity observed for nearby salt bridge

118 mutations $^{45-47}$ (Fig. 2c).

119 Interface II (Fig. 2a,b, $280 \AA^{2}$ BSA) is stabilized by both hydrophobic

120 (Hsp90 Y61,L64:Hsp70V163,1164) and polar interactions (Hsp90 R60,Y61: Hsp70 ${ }^{\text {D160}) . ~}$

121 Importantly, it defines the Hsp90 ATP-like position/orientation of the Hsp90NTD with

122 respect to the Hsp90 ${ }_{\mathrm{MD}}$, explaining the observation that Hsp70 accelerated Hsp90

123 ATPase activity ${ }^{48}$. Consistent with the significance of Interface II, mutation of the three 
124 Hsp90 interface residues $\left(\mathrm{Hsp} 90^{\mathrm{R} 60, \mathrm{Y} 61, \mathrm{~L} 64}\right)$ showed marked yeast growth defects at

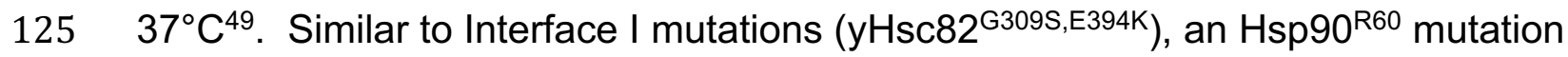

$126\left(\mathrm{yHsc82} 2^{\mathrm{R} 46 \mathrm{G}}\right)$ displayed reduced $\mathrm{Hsp} 70$ interaction, inviability at $37^{\circ} \mathrm{C}$, and reduced v-

127 Src activity (Extended Data Fig. 10). Lastly, sequence alignments of Hsp90/Hsp70

128 homologs/paralogues showed that Interface I \& II residues are generally conserved,

129 suggesting a universal Hsp70-Hsp90 binding strategy across species ${ }^{47,50}$ and

130 organelles ${ }^{51,52}$ (Extended Data Fig. 11,12).

131 As expected from Hsp90 ${ }^{\text {D93N }}$, both Hsp90 ${ }_{\text {NTD }}$ ATP-binding pockets are empty with their

132 lids open. The Hsp90ANTD and Hsp90B ${ }_{N T D S}$ closely resemble the structure of an apo

133 Hsp90 ${ }_{\text {NTD }}$ fragment ${ }^{53}$ (RMSD of 0.43 and $0.35 \AA$ to $3 \mathrm{TOH}$, respectively, Extended Data

134 Fig. 13a). The ATP pocket lid and the first $\alpha$-helix form a novel dimerization interface

$135\left(512 \AA^{2}\right.$ BSA, Fig. 1d). The two Hsp70 ${ }_{\text {NBDS }}$ clearly have ADP bound and are similar to the 136 ADP-bound Hsp70 NBD crystal structure ${ }^{54}$ (Ca-RMSD of $0.50 \AA$ (Hsp70C) and $0.53 \AA$

137 (Hsp70S) to 3AY9, respectively) (Fig. 2a, Extended Data Fig. 14a,b).

138 Coordination between the Hsp90/Hsp70 ATPase cycles is required for forming the

139 loading complex. The Hsp90ATP conformation is incompatible as closure of the

$140 \mathrm{Hsp90}{ }^{\text {ATP }}$ ATP pocket lid would clash with the Hsp70NBD (Extended Data Fig. 13b,c).

141 Thus, ATP binding to Hsp90 would be expected to accelerate loss of the bound Hsp70s.

142 Furthermore, the Hsp70ATP conformation is incompatible with the loading complex, as

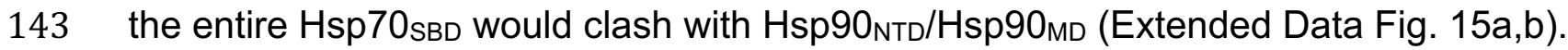

144 For Hsp70 to reenter its ATP cycle, it must first leave Hsp90, thus nucleotide exchange

145 on Hsp70 likely times its dissociation. Notably, in the complex Hsp70 NBD-IIA deviates

146 from the crystal structure (Extended Data Fig. 14a,b), likely explaining the weak Hsp70

147 nucleotide exchange activity provided by Hsp90 during the GR-chaperoning cycle ${ }^{55}$.

148 The canonical nucleotide exchange factor (NEF) binding sites ${ }^{56}$ on Hsp70NBD-IIB remain

149 available (Extended Data Fig. 16a,b), explaining how the NEF Bag-1 can accelerate GR

150 maturation ${ }^{55}$. 


\section{Hop interacts extensively with all components in the loading complex}

153 The cochaperone Hop is well conserved in eukaryotes and facilitates GR maturation in

154 vivo ${ }^{57}$ and in vitro ${ }^{14}$. Hop is thought to bring Hsp90 and Hsp70 together using its three

155 TPR domains that bind the EEVD C-termini on both Hsp90 and Hsp7058-60. Despite

156 using the full-length Hop construct, only three C-terminal domains (Hop TPR2A, HopTPR2B,

157 and HopDP2) are observed (Fig. 1e,f). Importantly, these three domains are necessary

158 and sufficient for full GR activation ${ }^{61,62}$. Hop wraps around much of the loading complex,

159 with extensive interactions made by HopTPR2A and HopDP2, demonstrating a far more

160 integral role than anticipated (Fig. 3a,c).

161 The structure of Hop ${ }^{\text {TPR2A }}-\mathrm{Hop}^{\text {TRP2B }}$ closely matches the yeast crystal structure ${ }^{61}$ (Ca-

162 RMSD of $1.47 \AA$ to $3 \cup Q 3$, Extended Data Fig. 17a,c,d), including the conserved

163 electrostatic network ( $\mathrm{Hop}^{\mathrm{Y3} 34, \mathrm{R} 389, \mathrm{E} 385, \mathrm{~K} 388}$ ) that defines the unique inter-domain angle

164 (Extended Data Fig. 18c-e). Focused maps revealed that the HopTPR2A and HopTPR2B

165 are bound to the EEVD termini of Hsp90 and Hsp70, respectively (Extended Data Fig.

166 19c-e,20a-d). Although the density for the remaining Hsp70 and Hsp90 tails are

167 missing, our structural modeling suggested the connectivity (Extended Data Fig. 21a,b).

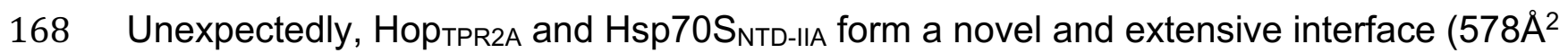

169 BSA) composed largely of polar interactions (Fig. 3a,b and Extended Data Fig. 19a-c,f).

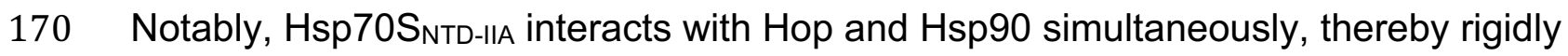

171 positioning Hop with respect to Hsp90. Although HopTPR2B was in close proximity ( 6 $\AA$ )

172 to Hsp90 ${ }_{\mathrm{MD}}$ no major contacts were observed (Fig. 3c). However, the low-resolution

173 Hsp90:Hop cryo-EM structure ${ }^{63}$ (Extended Data Fig. 22a) and previous studies ${ }^{61,64}$

174 show that Hop

175 may first prepare $\mathrm{Hsp} 90$ for $\mathrm{Hsp} 70$ and client interaction, and subsequently rearrange

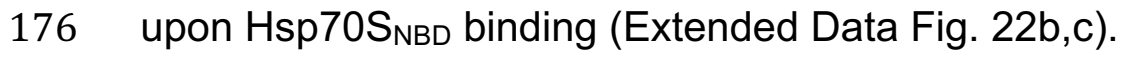

177 HopDP2 makes extensive interactions with both Hsp90 protomers at Hsp90ACTD and

$178 \mathrm{Hsp} \mathrm{B}_{\mathrm{MD}}$, thereby defining and maintaining the semi-closed Hsp90 conformation within

179 the loading complex (Fig. 3a). Interestingly, conserved client-binding residues on Hsp90

180 are repurposed for HopDP2 binding (Fig. 3d). Supporting our observations, Hsp90

181 mutations which would destabilize the HopDP2-Hsp90A interface (yHsp82 ${ }^{\text {W585T,M593T }}$ 


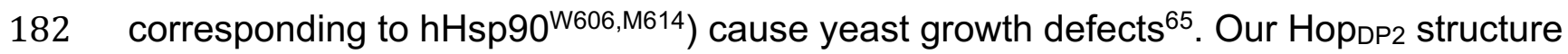

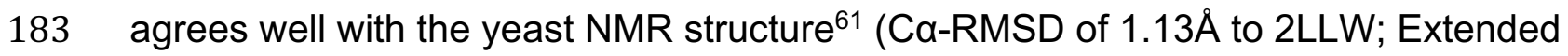

184 Data Fig. 17b), adopting a hand-like $a$-helical structure, with many of its core

185 hydrophobic sidechains exposed in the "palm" of the "hand" (Fig. 3d). Importantly, this

186 hydrophobic palm is continuous with the client binding surface provided by the lumen

187 between the Hsp90 protomers, augmenting the Hsp90A $\mathrm{A}_{a m p h i-\alpha}$ with a stronger and

188 extensive hydrophobic binding capability.

190 GR is unfolded, threaded through the Hsp90 lumen and bound by Hsp90, Hop and 191 Hsp70

192 In the high-resolution map, a strand of density can be seen passing through the Hsp90 193 lumen (Extended Data Fig. 23b,c). In the low-pass filtered map, this density connects to 194 the globular part of GR on one side of Hsp90 (Extended Data Fig. 24a,b). On the other 195 side, a GR helix is surprisingly cradled in the HopDP2 hydrophobic palm and the rest of

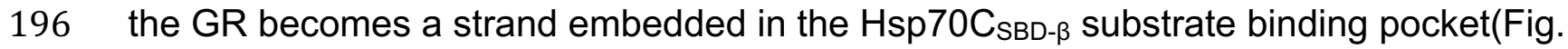
197 4a-d and Extended Fig. 25d,26e). Thus, GR is partially unfolded and threaded through 198 the Hsp90 lumen, reminiscent of how the CDK4 kinase was unfolded ${ }^{66}$ by the fully 199 closed Hsp90 ATP. To test this unexpected client:cochaperone interaction, we substituted $200 \mathrm{Hop}^{\mathrm{Q} 512}$ in HopDP2 which is close to, but not directly interacting with, the GR helix with 201 the photo-reactive unnatural amino acid p-benzoyl-phenylalanine (Extended Data Fig. 202 27b). In support of our structure, GR and Hop become photo-crosslinked (Extended 203 Data Fig. 27a,C). Additionally, mutation of Hop ${ }^{L 508 A}$ (L553 in yeast, Sti1) which is in the 204 hydrophobic palm that directly interacts with GR (Extended Data Fig. 28a), completely 205 abrogated GR function in vivo ${ }^{61}$.

206 Despite extensive 3D classifications, the main body of GR remained at low resolution. 207 Nonetheless, the Hsp90 ${ }_{\text {MDS }}$ from each protomer and the Hsp90B amphi- $\alpha_{\text {clearly contact }}$ 208 GR (Extended Data Fig. 23b-d, 24b,c). Hsp90 residues previously found ${ }^{34,36,45,46,67}$ to 209 impact GR maturation are highlighted in Fig. 4e. The exposed Hsp90A ${ }^{\text {W320,F349 }}$ directly 210 contacts GR in both the loading complex (Fig. 4e, Extended Data Fig. 24c), and the 211 maturation complex (Noddings et al., 2020). Notably, Hsp90 W320 (W300 in yHsp82) is an 
212 important binding residue exploited by both clients and cochaperones. Not only does it

213 interact with GR and HopDP2 (Fig. 3d), but also with another cochaperone Aha1 ${ }^{68}$.

214 Supporting its broad functional importance, numerous studies have reported effects of

215 Hsp90A $^{\text {W320 }}$ mutations on GR activation ${ }^{34,49,69}$.

\section{Discussion}

218 Our client-loading complex structure provides the first view of how Hsp70, Hsp90 and

219 Hop work together to chaperone a client. Several features were unexpected: 1) two

220 Hsp70s bind the Hsp90 dimer, one delivers client and the second scaffolds Hop. 2) Hop

221 interacts extensively with all components, including GR, going well beyond the

222 anticipated TPR-EEVD interactions. 3) Together Hop:Hsp90 and Hsp70:Hsp90

223 interactions define the Hsp90 conformation poising it for both client binding, and

224 ultimately for ATP hydrolysis and client activation. 4) Hsp90 repurposes one side of its

225 client-binding sites to bind Hop ${ }_{\mathrm{DP} 2}$, which in turn augments the Hsp90 lumenal client-

226 binding site, facilitating client-loading from Hsp70 (Fig. 5a).

227 The loading complex provides an extraordinarily extended client-binding pocket, with a

228 large and very adaptable surface for client recognition (Fig. 4b and Extended Data Fig.

229 23d): (1) Hsp70 binds a hydrophobic strand, (2) HopDP2 binds a

230 hydrophobic/amphipathic helix, (3) the remaining part of the Hsp90A $\mathrm{A}_{\text {amphi-a }}$ provides

231 polar interactions, (4) the Hsp90Bamphi-a provides a hydrophobic surface, and (5) the

$232 \mathrm{Hsp90A/B}$ lumen provides a combination of hydrophobic and polar interactions. Not only

233 is the loading complex lumen spacious enough to bind a strand (as shown here) or

234 intact helix (Extended Data Fig. 29), but the flexible positioning of the Hsp70C SBD $_{\text {and }}$

235 the dynamic, adaptable conformation of the Hsp90 amphi-a allow even broader flexibility for

236 client recognition (Fig. 4b,5a).

237 Which GR segment is captured in the loading complex lumen? The GR maturation

238 complex structure unambiguously demonstrates that GR's pre-Helix 1 region (GR ${ }^{517-533}$,

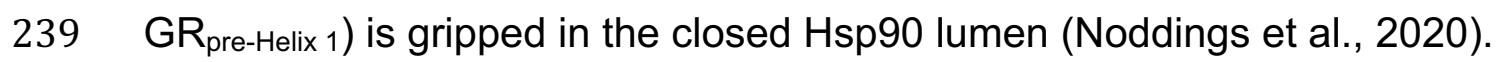


240 Reexamination of previous Hsp70-GR HDX-MS data ${ }^{14}$ reveals that only GR pre-Helix 1

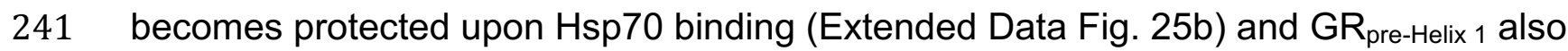
242 contains high-scoring predicted Hsp70 binding sites (GR ${ }^{518-524}$, Extended Data Fig.

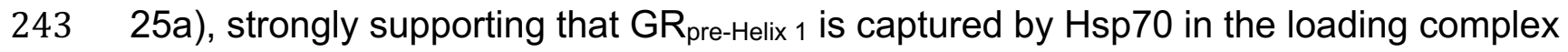

244 (Extended Data Fig. 25b,c).. Moreover, a previous optical-tweezer study ${ }^{70}$ demonstrated

245 that $\mathrm{GR}_{\text {Helix } 1}$ is readily detached, correlating with ligand binding loss. Together, this

246 implies that perturbing $\mathrm{GR}_{\text {Helix } 1}$ by Hsp70 or the loading complex leads to loss of GR

247 ligand binding. Altogether, we propose the following pathway for loading complex

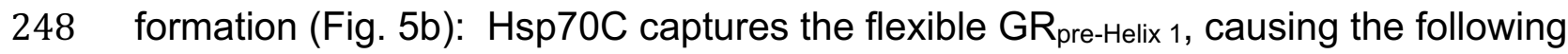

249 dynamic helix-strand motif to detach thereby destabilizing the GR ligand-binding pocket.

250 Hsp70C then delivers the partially unfolded GR to Hop:Hsp70S:Hsp90. In the resultant

251 loading complex, GR is further unfolded via engagement of GR $R_{\text {Helix }}$ 's LXXLL motif with

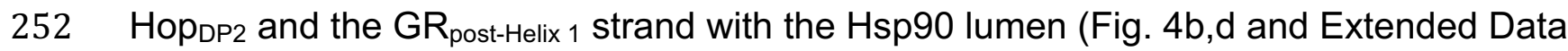

253 Fig. 28), suppressing any possible ligand binding. The rest of GR remains globular and

254 is only loosely associated with the distal surface of Hsp90.

255 How does the loading complex progress to the maturation complex-a process

256 requiring Hsp90 ATP hydrolysis and release of Hop and both Hsp70s? The one-Hsp70

257 loading complex suggests that the process is asymmetric and sequential, with the loss

258 of Hsp70C occurring first, while Hsp90 ATP hydrolysis drives the release of the more

259 tightly engaged Hsp70S-Hop. Schematically shown in Fig. 5b, we propose that a

260 combination of Hsp90's ATP binding and NEF activities promotes Hsp70C to release

261 GR and exit the complex. This leaves GR engaged with HopDP2 and the Hsp90 lumen,

262 minimizing reformation of an Hsp70:GR complex or premature release. Lastly, as

263 discussed in detail in Noddings et al., the conversion of the semi-closed Hsp90 in the

264 loading complex to the fully closed $\mathrm{Hsp} 90^{\mathrm{ATP}}$ in the maturation complex may serve as a

265 driving force for client remodeling and hence activation.

266 Contrary to kinases utilizing a dedicated cochaperone (Cdc37), GR uses a generalized

267 chaperone (Hsp70) and cochaperone (Hop) for loading Hsp90 clients, making the

268 principles learned here broadly applicable to other clients. Although Hop is absent in

269 bacteria and organellar compartments, Hsp70s are present and the client-binding 


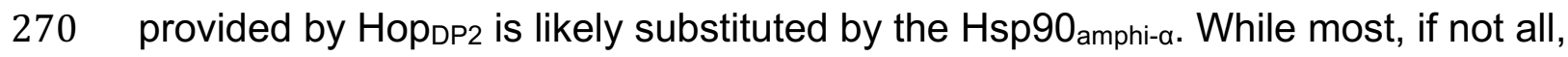

271 proteins engage with Hsp70 at least during initial folding, only a subset are Hsp90

272 clients. Ultimately, client properties must dictate this selectivity. Rather than an overall

273 client property such as stability, our loading complex structure suggests a more

274 nuanced balance of three effects: 1) the probability of partial unfolding fluctuations in the

275 client, 2) the ability of Hsp70 to capture a transiently exposed site, and 3) the likelihood

276 that further unfolding events would uncover adjacent client regions that can be captured

277 by Hop $2 / H s p 90$. Experiments to test these general principles can now be designed to

278 predict and identify potential Hsp90/Hsp70 clients.

\section{Materials and Methods}

\section{Protein purification}

282 All recombinant chaperone proteins of Hsp90a, Hop, and Hsp70 (from human), and ydj1

283 (yeast Hsp40) were in general expressed and purified as described previously ${ }^{14}$ but with 284 minor modifications as described below. Proteins were expressed in E coli BL21 star

285 (DE3) strain. Cells were grown in $\mathrm{TB}$ at $37^{\circ} \mathrm{C}$ until $\mathrm{OD}_{600}$ reached 0.8. Protein expression was induced with $0.5 \mathrm{mM}$ IPTG for 16 hours at $16^{\circ} \mathrm{C}$. Cells were harvested by centrifugation at $4000 \mathrm{xg}$ for 15 minutes and resuspended in lysis buffer ( $50 \mathrm{mM}$ Tris $\mathrm{pH}$ 7.5, $500 \mathrm{mM} \mathrm{KCl}, 10 \mathrm{mM}$ Imidazole, 5mM $\beta M E)$. A protease inhibitor cocktail (Roche) was then added. Cells were lysed by an Emulsiflex system (Avestin). Lysates were cleared by centrifugation at $20,000 \mathrm{rpm}$ for 1 hour at $4^{\circ} \mathrm{C}$ and the soluble fraction was affinity purified by gravity column with Ni-NTA affinity resin (QIAGEN). The protein was eluted by $50 \mathrm{mM}$ Tris $\mathrm{pH} 8,50 \mathrm{mM} \mathrm{KCl}$, and $5 \mathrm{mM} \mathrm{\beta ME}$. The $6 x$-His-tag was removed with TEV protease and dialysis into low-salt buffer overnight (50 mM Tris pH 8,

$29450 \mathrm{mM} \mathrm{KCl}$, and $5 \mathrm{mM} \beta M E)$. The cleaved protein was purified with MonoQ 10/100 GL 295 (GE Healthcare), an ion-exchange column with $30 \mathrm{mM}$ Tris pH 8, $50 \mathrm{mM} \mathrm{KCl}, 5 \mathrm{mM}$ $296 \beta \mathrm{ME}$ and eluted with a linear gradient of $50-500 \mathrm{mM} \mathrm{KCl}$. Fractions with the target 297 protein were then pooled and concentrated for final purification of size exclusion in $\mathbf{3 0}$ mM HEPES pH 7.5, $50 \mathrm{mM} \mathrm{KCl,} 2$ mM DTT, and 10\% Glycerol using a Superdex S200 
16/60 (GE Healthcare) or Superdex S75 16/60 (GE Healthcare). The peak fractions

300 were pooled, concentrated to $\sim 100-150 \mu \mathrm{M}$ or greater, snap-frozen in liquid nitrogen, 301 and stored in aliquots at $-80^{\circ} \mathrm{C}$. MBP-GRLBD (F602S) was expressed and purified as 302 described previously ${ }^{14}$. Note that for complex preparation, Hsp70 from Sf9 cell source 303 was used with purification as described previously ${ }^{14}$. The Hop construct for the 304 crosslinking experiment was obtained using quick-change at Q512 to the Amber codon.

305 The construct was expressed in E. coli BL21 DE3 cells containing the pEVOL-pBpF 306 plasmid $^{71}$ distributed by the lab of Peter Schultz through Addgene (\#31190). Cells were

307 grown in terrific broth to an $\mathrm{OD}_{600}$ of 0.6 . For induction, arabinose $(0.02 \%)$, IPTG (1 $308 \mathrm{mM}$ ), and p-benzoylphenylalanine (pBpa; $0.7 \mathrm{mM}$ ) was added, and expression was 309 carried out overnight at $16^{\circ} \mathrm{C}$. Cell harvesting, lysis, and Ni-NTA purification was 310 performed as described above.

\section{Complex preparation}

313 Using reaction buffer containing $50 \mathrm{mM} \mathrm{HEPES} \mathrm{pH} \mathrm{7.5,} 50 \mathrm{mM} \mathrm{KCl}$, and $2 \mathrm{mM} \mathrm{DTT}, 10$ $314 \mu \mathrm{M}$ Hsp90 dimer of D93N mutant, $10 \mu \mathrm{M}$ Hop, $15 \mu \mathrm{M}$ Hsp70, $4 \mu \mathrm{M}$ Hsp40, and $20 \mu \mathrm{M}$ 315 MBP-GRLBD were incubated with $5 \mathrm{mM} A T P / \mathrm{MgCl}$ for 1 hour at room temperature. The 316 complex was purified and analyzed by SEC-MALS with a Wyatt $050 S 5$ column on an 317 Ettan LC (GE Healthcare) in a running buffer containing $50 \mathrm{mM} \mathrm{HEPES,} 50 \mathrm{mM} \mathrm{KCl}, 5$ $318 \mathrm{mM} \mathrm{MgCl} 2,2 \mathrm{mM}$ DTT, $200 \mu \mathrm{M}$ ADP and 0.01\% Octyl $\beta$-D-glucopyranoside ( $\beta$-OG);

319 Molecular weights were determined by multiangle laser light scattering using an in-line

320 DAWN HELEOS and Optilab rEX differential refractive index detector (Wyatt

321 Technology Corporation). Once eluted, fractions containing the GR-loading complex

322 were immediately crosslinked with $0.02 \%$ glutaraldehyde for 20 minutes at room

323 temperature and quenched with $20 \mathrm{mM}$ Tris pH 7.5. Fractions containing the GR-

324 loading complex were separately snap-frozen in liquid nitrogen, and stored in aliquots at $325-80^{\circ} \mathrm{C}$. 
328 To ensure that Hop crosslinks the bound segment in the context of the loading complex, 329 crosslinking reactions were performed immediately after the complex was fractionated 330 from SEC (see the above complex preparation section). Using a UV-transparent, 96-

331 well microplate (Corning) as a fraction collector, the whole fractions of the eluted GR-

332 loading complex were subjected to UV exposure using an agarose gel imaging system

333 (Enduro GDS Imaging System). Samples were irradiated for 60 mins in total. To prevent

334 overheating, the 96-well plate was placed on a shallow plate filled with constantly

335 refreshed ice water during the time course of the exposure. SDS-PAGE was used to

336 analyze cross-linked product, followed by Western plot transfer to nitrocellulose and

337 probed with an MBP antibody (New England BioLabs) (Extended Data Fig. 27).

\section{Cryo-EM sample/grid preparation and data acquisition}

340 The flash-frozen fractions of the loading complex were thawed and concentrated to 0.7 -

$3410.8 \mu \mathrm{M}$. About $2.5 \mathrm{uL}$ of the complex sample was applied onto a glow-discharged, holey

342 carbon grid (Quantifoil R1.2/1.3, Cu, 400 mesh), blotted by Vitrobot Mark IV (FEI) for 8-

34314 seconds at $10^{\circ} \mathrm{C} / 100 \%$ humidity, and plunge-frozen in liquid ethane. Four data

344 collections were made using Titan Krios (Thermo Fisher Scientific) equipped with K2

345 camera (Gatan K2). SerialEM ${ }^{72}$ was used for all the data collections with parameters as

346 described in Extended Data Table 1.

Image processing

349 Movies were motion-corrected using MotionCor2 ${ }^{73}$, in which the unweighted summed

350 images were used for CTF estimation using CTFFIND $4^{74}$, and the dose-weighted

351 images were used for image analysis with RELION ${ }^{42}$ throughout. The initial model of the

352 loading complex was obtained from a small data collection (Extended Data Fig. 2a).

353 Particles were picked from the small data collection using Gautomatch

354 (https://www2.mrc-Imb.cam.ac.uk/research/locally-developed-software/zhang-software/)

355 without template and subjected to reference-free 2D classification (Class2d) using

356 RELION. 2D class averages with proteinaceous features were selected for 3D 
357 classification (Class3d) using RELION. For Class3d, the reference model was

358 generated using the semi-open conformation Hsp90 from the Hsp90:Hop cryoEM

359 structure $^{63}$ (Extended Data Fig. 22). Among eight classes, one class ( $8 \AA$ resolution)

360 showed recognizable shapes of the protein components, although the class is

361 drastically different from the initial model. This low-resolution reconstruction of the

362 loading complex was then used as an initial reference model for the following image

363 analysis that achieved high-resolution.

364 The procedure to obtain the high-resolution reconstruction is shown schematically in

365 Extended Data Fig. 2b. Particles were picked from all dose-weighted micrographs using

366 Gautomatch with the low-resolution reconstruction as a template. Without using

367 Class $2 d$, the extracted, binned $4 \times 4$ particles $\left(4.236 \AA\right.$ pixel $\left.^{-1}\right)$ were subjected to

368 RELION Class3d (4 classes) to sort out "empty" or non-proteinaceous particles.

369 Particles from the selected class were re-centered and re-extracted to binned $2 \times 2$

$370\left(2.118 \AA\right.$ pixel $\left.^{-1}\right)$ for another round of Class $3 d$. Note that a low-resolution $(\sim 7.5 \AA)$

371 reconstruction of a one-Hsp70 loading complex was obtained among the 4 classes. The

372 selected class that contains 636,056 particles of the two-Hsp70 loading complex was

373 3D auto-refined (Refine3d) into a single class (consensus class). The set of particles

374 are then used for further global classification and focused classification (described

375 below). For global classification, another round of masked Class3d (4 classes) was

376 performed without alignment, followed by masked Refine $3 d$ using unbinned particles

$377\left(1.059 \AA\right.$ pixel $\left.^{-1}\right)$. Finally, 85,619 particles from the highest resolution, the two-Hsp70

378 loading class were further subjected to multiple rounds of per-particle CTF/beam-tilt

379 refinement until the gold-standard resolution determined by Refine3d no longer

380 improved. The overall gold-standard resolution for the global reconstruction of the

381 loading complex is $3.57 \AA$ (Extended Data Fig. 3). Local resolution was estimated using

382 the RELION (Extended Data Fig. 3a).

383 The loading complex presents conformational heterogeneity at all regions of the 384 complex, in particular at the Hop TPR2A-TPR2B (Extended Data Fig 18. a-c). Starting from 385 the consensus class containing 636,056 binned $2 \times 2$ particles $\left(2.118 \AA\right.$ pixel $\left.^{-1}\right)$, masks 386 at various GR-loading complex regions were used for focused classification with signal 
387 subtraction (Focused-class3d; Extended Data Fig. 4b). A pipeline to obtain the best 388 reconstruction for each masked region is outlined in Extended Data Fig. 4a. For each 389 masked region, Class $3 d$ without alignment was performed, followed by Refine $3 d$ using 390 unbinned particles $\left(1.059 \AA\right.$ pixel $\left.^{-1}\right)$. For each Class $3 d$ job, parameters of number of 391 requested classes $(K=6,8,10,12,14)$ and $\operatorname{Tau}(T=10,20,30,40)$ were scanned. For each

392 masked region, the reconstruction that results in the highest resolution determined by

393 the gold-standard FSC of the Refine3d job was selected for each masked region. Using

394 the unbinned particles, another round of Focused-class $3 d$ was performed with the 395 similar procedure described for the previous round. Parameters were scanned in a 396 similar manner but with smaller requested classes $(K=2,3,4,5)$. Finally, the selected

397 Focused-class $3 d$ job was subjected to multiple rounds of per-particle CTF/beam-tilt 398 refinement. The overall resolution of the reconstruction for each masked region is 399 determined by the gold-standard FSC and as denoted in the FSC plots in Extended 400 Data Fig. 4b and Extended Data Table 2. The focused maps showed much better 401 atomic details than the global reconstruction at all regions, and hence were used for 402 model building and refinement.

\section{Model Building and refinement}

405 Model building and refinement was carried out using Rosetta throughout. All the 406 components of the loading complex had crystal structures or close homologous 407 structures (from yeast) available. Details of how the starting, unrefined atomic model for 408 each component was obtained are described below. For Hsp90, the starting model was 409 assembled from the crystal structure of human Apo-Hsp90 NTD $\left._{\text {(PDB ID: } 3 T 0 H}\right)^{53}$ and the 410 cryo-EM structure of the Hsp90MD-стD from the GR-maturation complex (Noddings et al., 411 2020). Many crystal structures of Hsp70 NBD were available. As Potassium and 412 Magnesium ions were used in the buffer for complex preparation and there is density 413 accounted for them in our focused map (Extended Data Fig. 14), the ADP state $414 \mathrm{Hsp70}_{\mathrm{NBD}}$ structure crystal structure (PDB ID: 3AY9) ${ }^{54}$ that has Potassium and 415 Magnesium ions to coordinate ADP was used as an starting model. For the Hsp70sBD, 416 the human Hsp70 crystal structure (PDB ID: 4PO2) ${ }^{75}$ was used as a starting model. For 
417 Hop, the crystal structures ${ }^{61}$ of the Hop TPR2A-TPR2B (PDB ID: 3UQ3) and the Hop DP2 (PDB 418 ID: 2LLW) from yeast were used as initial templates with the alignments obtained from $419 \mathrm{HHpred} \mathrm{server}^{76}$ (Extended Data Fig. 17). The insertion in the threaded Hop model was 420 completed using RosettaCM guided by the cryo-EM density ${ }^{77}$. The resulting completed

421 models of HopTPR2A and HopTPR2B showed a high resemblance to their structures 422 determined by NMR individually (Extended Data Fig. 17c,d). The sequence of the

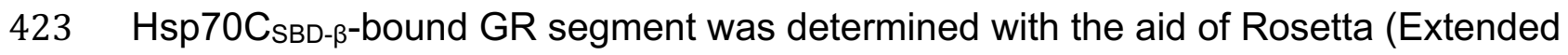
424 Data Fig. 26). Two 7-residue GR segments (SIVPATL and IVPATLP) of a continuous 425 sequence (residues 518-525; note that residue 518 in the native GR sequence is $T$, not $426 \mathrm{~S}$ ) in the pre-Helix 1 regions are predicted to be Hsp70 binding sites by two state-of-the427 art algorithms (BiPPred ${ }^{78}$ and ChaperISM $^{79}$ ). Structural modeling of the two GR 428 peptides in the templates ${ }^{80}$ (PDB IDs: 4EZZ, 4EZT, and 4EZQ) with "reverse" binding

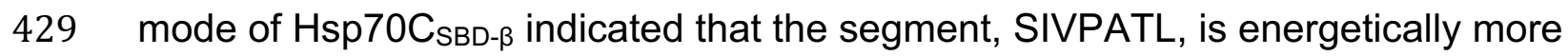
430 favored (Extended Data Fig. 26a-c).

431 Using the high-resolution information acquired from focused classification/refinement, 432 the starting models were refined separately into the individual focused maps (Extended 433 Data Fig 4.). Model overfitting was monitored and harbored using the half-map 434 approach as previously described ${ }^{81,82}$, in which one-half map from RELION Refine3d 435 was used for density-guided refinement while the other half map was used for 436 validation. Rosetta fragment-based iterative refinement protocol ${ }^{82}$ was used to refine the 437 models throughout. Based on the high-resolution focused maps, the refinement tasks 438 were split into (1) Hsp90A:Hsp70C, (2) Hsp90B:Hsp70S (Extended Data Fig. 4,8), (3) 439 Hsp70S:HoptPR2A (Extended Data Fig 4,19) and (4) Hsp90AB

$440 \quad \beta:$ Hop $_{\mathrm{DP} 2}: \mathrm{GR}_{\mathrm{Helix} 1}$ (Extended Data Fig. 4,23). To model the GR segment threaded 441 through the lumen of Hsp90, the GR $R_{\text {Helix } 1}$ motif (residues 528-551) was first segmented

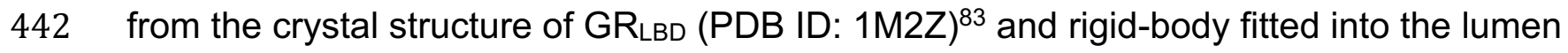
443 density. The $\mathrm{GR}_{\text {Helix } 1}$ segment was then rebuilt and refined using Rosetta fragment-

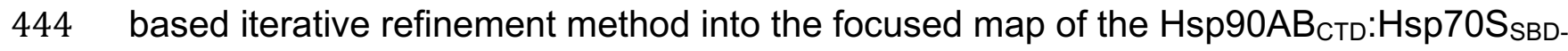
$445 \quad \beta: H_{0 p}{ }_{D 2}: R_{\text {Helix } 1}$ (Extended Data Fig. 23). The remaining, globular portion of GR $R_{L B D}$ 446 was rigid-body fitted initially using Chimera. The placement was further refined, guided 447 by (1) the connectivity to the GR $\mathrm{Relix}_{1}$ motif and (2) GR's interaction with Hsp90 MD in the 
448 maturation complex. The docked $\mathrm{GR}_{\mathrm{LBD}}$ was then energy minimized in Rosetta guided

449 by low-pass filtered cryo-EM map. Finally, the connectivities of the $\mathrm{N}$-terminal end of the

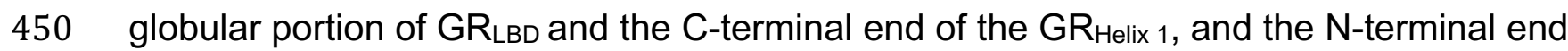

451 of the $\mathrm{GR}_{\text {Helix } 1}$ motif and the C-terminal end of the Hsp70-bound GR pre-Helix $1_{1}$ portion

452 (Extended Data Fig. 26d-f) were built using RosettaCM. The final model of the loading

453 complex was assembled and refined into the high-resolution global construction,

454 followed by B-factor refinement (Extended Data Fig. 5).

455 Structural modeling was used to ensure and suggest the connectivities of the EEVD

456 tails of Hsp90/Hsp70 to the bound TPR domains of Hop (Extended Data Fig. 21). For

457 each protomer of $\mathrm{Hsp90,} 40$ residues of the tail were modeled using RosettaCM to

458 connect the bound Hsp90 EEVD fragment and the very C-terminal helix in the

459 Hsp90CTD. Similarly, for Hsp70C, the remaining residues were built, including a

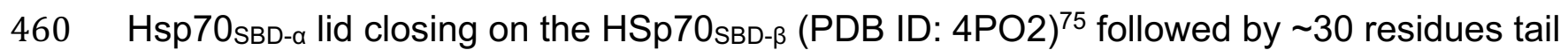

461 residues to the Hsp70 EEVD fragment bound to HopтPR2B.

\section{In vivo yeast Hsp90:Hsp70 interaction assay}

464 Hsc82 plasmids expressing untagged or His-Hsc82 were expressed in yeast strain 465 JJ816 (hsc82::LEU2 hsp82::LEU2/YEp24-HSP82). His-Hsc82 complexes were isolated 466 as described ${ }^{84}$. Antibodies against the last 56 amino acids of Ssa1/2 were a gift from 467 Dr. Elizabeth Craig (University of Wisconsin). The Sti1 peptide antisera was raised 468 amino acids 91-108. His-Hsc82 was detected using an anti-Xpress antibody, which 469 recognizes sequences near the $6 \mathrm{X}$-His tag at the amino-terminus. The plasmid pBv470 src, which expresses v-src under the GAL1 promoter, and the corresponding empty 471 vector pB656 were a gift from Frank Boschelli ${ }^{85}$. The R46G and K394E mutations were 472 isolated in a genetic screen as described ${ }^{47}$. 


\section{Data availability}

475 The electron microscopy maps and atomic model have been deposited into the Electron 476 Microscopy Data Bank (EMDB) and the Protein Data Bank (PDB). The accession codes 477 for the GR-loading complex are EMD-23050 and 7KW7. Focused maps used for model 478 refinements were also deposited with accession codes denoted in Extended Data Table 4792 (EMD-23051, EMD-23053, EMD-23054, EMD-23055, EMD-23056).

\section{Declaration of interests}

482 The authors declare no competing interests.

\section{Author contributions}

485 R.Y.-R.W. performed the research and drafted the manuscript. D.A.A. supervised the 486 research. E.K. trained R.Y.-R.W. for the biochemistry of the GR reconstitution system. 487 A.G.M. trained R.Y.-R.W. for cryo-EM operation and data acquisition. J.L.J. carried out 488 in vivo yeast experiments. R.Y.-R.W., C.M.N., and D.A.A. wrote the manuscript with 489 input from all authors.

\section{Acknowledgements}

492 We thank members of the Agard Lab for helpful discussions. We thank Dr. Tristan W.

493 Owens for advising the photoreactive crosslinking experiment. We thank Michael 494 Braunfeld, David Bulkley, Glenn Gilbert, Eric Tse, and Zanlin Yu from the W.M. Keck 495 Foundation Advanced Microscopy Laboratory at the University of California San 496 Francisco (UCSF) for maintaining the EM facility and help with data collection. We thank 497 Matt Harrington and Joshua Baker-LePain for computational support with the UCSF 498 Wynton cluster. R.Y.-R.W. was a Howard Hughes Medical Institute Fellow of the Life 
Sciences Research Foundation. The work was supported by funding from Howard

500 Hughes Medical Institute (D.A.A.) and NIH grants R35GM118099 (D.A.A.),

501 S10OD020054 (D.A.A.), S10OD021741 (D.A.A.), and R01GM127675 (J.L.J.).

502

\section{References}

5041 Kim, Y. E., Hipp, M. S., Bracher, A., Hayer-Hartl, M. \& Hartl, F. U. Molecular

$505 \quad$ chaperone functions in protein folding and proteostasis. Annu Rev Biochem 82 ,

506 323-355, doi:10.1146/annurev-biochem-060208-092442 (2013).

5072 Labbadia, J. \& Morimoto, R. I. The biology of proteostasis in aging and disease.

$508 \quad$ Annu Rev Biochem 84, 435-464, doi:10.1146/annurev-biochem-060614-033955

509 (2015).

$5103 \quad$ Genest, O., Wickner, S. \& Doyle, S. M. Hsp90 and Hsp70 chaperones:

511

512

5134 Moran Luengo, T., Mayer, M. P. \& Rudiger, S. G. D. The Hsp70-Hsp90

$514 \quad$ Chaperone Cascade in Protein Folding. Trends Cell Biol 29, 164-177,

515 doi:10.1016/j.tcb.2018.10.004 (2019).

5165 Schopf, F. H., Biebl, M. M. \& Buchner, J. The HSP90 chaperone machinery. Nat

$517 \quad$ Rev Mol Cell Biol 18, 345-360, doi:10.1038/nrm.2017.20 (2017).

$5186 \quad$ Taipale, M. et al. A quantitative chaperone interaction network reveals the 519 architecture of cellular protein homeostasis pathways. Cell 158, 434-448, 520 doi:10.1016/j.cell.2014.05.039 (2014).

5217 Taipale, M. et al. Quantitative analysis of HSP90-client interactions reveals

522

523

5248 Morishima, Y., Murphy, P. J., Li, D. P., Sanchez, E. R. \& Pratt, W. B. Stepwise

525

526

527

528

5299 Lorenz, O. R. et al. Modulation of the Hsp90 chaperone cycle by a stringent client $530 \quad$ protein. Mol Cell 53, 941-953, doi:10.1016/j.molcel.2014.02.003 (2014).

53110 Picard, D. et al. Reduced levels of hsp90 compromise steroid receptor action in

532 vivo. Nature 348, 166-168, doi:10.1038/348166a0 (1990). 
$53311 \quad$ Nathan, D. F., Vos, M. H. \& Lindquist, S. In vivo functions of the Saccharomyces $534 \quad$ cerevisiae Hsp90 chaperone. Proc Natl Acad Sci U S A 94, 12949-12956, 535 doi:10.1073/pnas.94.24.12949 (1997).

53612 Smith, D. F. \& Toft, D. O. Minireview: the intersection of steroid receptors with

13 Pratt, W. B. \& Toft, D. O. Steroid receptor interactions with heat shock protein molecular chaperones: observations and questions. Mol Endocrinol 22, 22292240, doi:10.1210/me.2008-0089 (2008).

541 and immunophilin chaperones. Endocr Rev 18, 306-360, doi:10.1210/edrv.18.3.0303 (1997).

54615 Rosenzweig, R., Nillegoda, N. B., Mayer, M. P. \& Bukau, B. The Hsp70

Kirschke, E., Goswami, D., Southworth, D., Griffin, P. R. \& Agard, D. A.

553 Glucocorticoid receptor function regulated by coordinated action of the Hsp90 and Hsp70 chaperone cycles. Cell 157, 1685-1697, doi:10.1016/j.cell.2014.04.038 (2014).

55418 Whitesell, L. \& Lindquist, S. L. HSP90 and the chaperoning of cancer. Nat Rev $555 \quad$ Cancer 5, 761-772, doi:10.1038/nrc1716 (2005).

55619 Trepel, J., Mollapour, M., Giaccone, G. \& Neckers, L. Targeting the dynamic HSP90 complex in cancer. Nat Rev Cancer 10, 537-549, doi:10.1038/nrc2887 (2010).

Lackie, R. E. et al. The Hsp70/Hsp90 Chaperone Machinery in

561 Neurodegenerative Diseases. Front Neurosci 11, 254, doi:10.3389/fnins.2017.00254 (2017). 
56923 Mayer, M. P. \& Gierasch, L. M. Recent advances in the structural and mechanistic aspects of Hsp70 molecular chaperones. J Biol Chem 294, 20852097, doi:10.1074/jbc.REV118.002810 (2019).

57224 Qi, R. et al. Allosteric opening of the polypeptide-binding site when an Hsp70 binds ATP. Nat Struct Mol Biol 20, 900-907, doi:10.1038/nsmb.2583 (2013).

57425 Zhuravleva, A., Clerico, E. M. \& Gierasch, L. M. An interdomain energetic tug-ofwar creates the allosterically active state in Hsp70 molecular chaperones. Cell 151, 1296-1307, doi:10.1016/j.cell.2012.11.002 (2012).

57726 Swain, J. F. et al. Hsp70 chaperone ligands control domain association via an allosteric mechanism mediated by the interdomain linker. Mol Cell 26, 27-39, doi:10.1016/j.molcel.2007.02.020 (2007).

58027 Kityk, R., Kopp, J., Sinning, I. \& Mayer, M. P. Structure and dynamics of the ATPbound open conformation of Hsp70 chaperones. Mol Cell 48, 863-874, doi:10.1016/j.molcel.2012.09.023 (2012).

58328 Bertelsen, E. B., Chang, L., Gestwicki, J. E. \& Zuiderweg, E. R. Solution conformation of wild-type E. coli Hsp70 (DnaK) chaperone complexed with ADP and substrate. Proc Natl Acad Sci U S A 106, 8471-8476, doi:10.1073/pnas.0903503106 (2009).

29 Krukenberg, K. A., Street, T. O., Lavery, L. A. \& Agard, D. A. Conformational dynamics of the molecular chaperone Hsp90. Q Rev Biophys 44, 229-255, doi:10.1017/S0033583510000314 (2011).

592

31 Street, T. O., Lavery, L. A. \& Agard, D. A. Substrate binding drives large-scale

Shiau, A. K., Harris, S. F., Southworth, D. R. \& Agard, D. A. Structural Analysis of E. coli hsp90 reveals dramatic nucleotide-dependent conformational rearrangements. Cell 127, 329-340, doi:10.1016/j.cell.2006.09.027 (2006). conformational changes in the Hsp90 molecular chaperone. Mol Cell 42, 96-105, doi:10.1016/j.molcel.2011.01.029 (2011). different types of client proteins. Mol Cell Biol 26, 8385-8395, 
60435 Meyer, P. et al. Structural and Functional Analysis of the Middle Segment of Hsp90: Implications for ATP Hydrolysis and Client Protein and Cochaperone Interactions. Molecular Cell 11, 647-658, doi:10.1016/s1097-2765(03)00065-0 (2003).

Genest, O. et al. Uncovering a region of heat shock protein 90 important for client binding in E. coli and chaperone function in yeast. Mol Cell 49, 464-473, doi:10.1016/j.molcel.2012.11.017 (2013).

Boysen, M., Kityk, R. \& Mayer, M. P. Hsp70- and Hsp90-Mediated Regulation of the Conformation of p53 DNA Binding Domain and p53 Cancer Variants. Mol Cell 74, 831-843 e834, doi:10.1016/j.molcel.2019.03.032 (2019).

61438 Dahiya, V. et al. Coordinated Conformational Processing of the Tumor Suppressor Protein p53 by the Hsp70 and Hsp90 Chaperone Machineries. Mol Cell 74, 816-830 e817, doi:10.1016/j.molcel.2019.03.026 (2019).

39 Moran Luengo, T., Kityk, R., Mayer, M. P. \& Rudiger, S. G. D. Hsp90 Breaks the Deadlock of the Hsp70 Chaperone System. Mol Cell 70, 545-552 e549, doi:10.1016/j.molcel.2018.03.028 (2018).

Tsuboyama, K., Tadakuma, H. \& Tomari, Y. Conformational Activation of Argonaute by Distinct yet Coordinated Actions of the Hsp70 and Hsp90 Chaperone Systems. Mol Cell 70, 722-729 e724, doi:10.1016/j.molcel.2018.04.010 (2018).

Obermann, W. M., Sondermann, H., Russo, A. A., Pavletich, N. P. \& Hartl, F. U. In vivo function of $\mathrm{Hsp} 90$ is dependent on ATP binding and ATP hydrolysis. $J$ Cell Biol 143, 901-910, doi:10.1083/jcb.143.4.901 (1998).

Scheres, S. H. RELION: implementation of a Bayesian approach to cryo-EM structure determination. J Struct Biol 180, 519-530, doi:10.1016/j.jsb.2012.09.006 (2012).

Morgner, N. et al. Hsp70 forms antiparallel dimers stabilized by post-translational modifications to position clients for transfer to Hsp90. Cell Rep 11, 759-769, doi:10.1016/j.celrep.2015.03.063 (2015).

Kityk, R., Kopp, J. \& Mayer, M. P. Molecular Mechanism of J-Domain-Triggered ATP Hydrolysis by Hsp70 Chaperones. Mol Cell 69, 227-237 e224, doi:10.1016/j.molcel.2017.12.003 (2018).

Bohen, S. P. \& Yamamoto, K. R. Isolation of Hsp90 mutants by screening for decreased steroid receptor function. Proc Natl Acad Sci U S A 90, 11424-11428, doi:10.1073/pnas.90.23.11424 (1993). 
63946 Nathan, D. F. \& Lindquist, S. Mutational analysis of Hsp90 function: interactions $640 \quad$ with a steroid receptor and a protein kinase. Mol Cell Biol 15, 3917-3925, 641 doi:10.1128/mcb.15.7.3917 (1995).

$64247 \quad$ Kravats, A. N. et al. Functional and physical interaction between yeast Hsp90

643

644

64548 Genest, O., Hoskins, J. R., Kravats, A. N., Doyle, S. M. \& Wickner, S. Hsp70 and 646

647

648 and Hsp70. Proc Natl Acad Sci U S A 115, E2210-E2219, doi:10.1073/pnas.1719969115 (2018).

49 Flynn, J. M. et al. Comprehensive fitness maps of Hsp90 show widespread Hsp90 of E. coli Directly Interact for Collaboration in Protein Remodeling. J Mol Biol 427, 3877-3889, doi:10.1016/j.jmb.2015.10.010 (2015).

65050 Genest, O., Hoskins, J. R., Camberg, J. L., Doyle, S. M. \& Wickner, S. Heat shock protein 90 from Escherichia coli collaborates with the DnaK chaperone system in client protein remodeling. Proc Natl Acad Sci U S A 108, 8206-8211,

652

653

654

51 Sung, N. et al. 2.4 A resolution crystal structure of human TRAP1NM, the Hsp90 paralog in the mitochondrial matrix. Acta Crystallogr D Struct Biol 72, 904-911, doi:10.1107/S2059798316009906 (2016).

65752 Sun, M., Kotler, J. L. M., Liu, S. \& Street, T. O. The endoplasmic reticulum (ER) chaperones BiP and Grp94 selectively associate when BiP is in the ADP conformation. J Biol Chem 294, 6387-6396, doi:10.1074/jbc.RA118.007050 (2019).

$53 \mathrm{Li}$, J. et al. Structure insights into mechanisms of ATP hydrolysis and the activation of human heat-shock protein 90. Acta Biochim Biophys Sin (Shanghai) 44, 300-306, doi:10.1093/abbs/gms001 (2012).

66454 Arakawa, A., Handa, N., Shirouzu, M. \& Yokoyama, S. Biochemical and structural studies on the high affinity of Hsp70 for ADP. Protein Sci 20, 1367-

66755 Kirschke, E., Roe-Zurz, Z., Noddings, C. \& Agard, D. The interplay between Bag1, Hsp70, and Hsp90 reveals that inhibiting Hsp70 rebinding is essential for Glucocorticoid Receptor activity. bioRxiv, doi:10.1101/2020.05.03.075523 (2020).

67056 Bracher, A. \& Verghese, J. The nucleotide exchange factors of Hsp70 molecular chaperones. Front Mol Biosci 2, 10, doi:10.3389/fmolb.2015.00010 (2015).

67257 Sahasrabudhe, P., Rohrberg, J., Biebl, M. M., Rutz, D. A. \& Buchner, J. The Plasticity of the Hsp90 Co-chaperone System. Mol Cell 67, 947-961 e945, doi:10.1016/j.molcel.2017.08.004 (2017). 
67558 Wegele, H., Haslbeck, M., Reinstein, J. \& Buchner, J. Sti1 is a novel activator of the Ssa proteins. J Biol Chem 278, 25970-25976, doi:10.1074/jbc.M301548200 (2003).

59 Chen, S. \& Smith, D. F. Hop as an adaptor in the heat shock protein 70 (Hsp70) and hsp90 chaperone machinery. J Biol Chem 273, 35194-35200, doi:10.1074/jbc.273.52.35194 (1998). Hsp70/Hsp90 interactions in protein folding. J Biol Chem 273, 3679-3686, doi:10.1074/jbc.273.6.3679 (1998).

68762 Rohl, A. et al. Hsp90 regulates the dynamics of its cochaperone Sti1 and the

Schmid, A. B. et al. The architecture of functional modules in the Hsp90 cochaperone Sti1/Hop. EMBO J 31, 1506-1517, doi:10.1038/emboj.2011.472 (2012). transfer of Hsp70 between modules. Nat Commun 6, 6655, doi:10.1038/ncomms7655 (2015).

69364 Lee, C. T., Graf, C., Mayer, F. J., Richter, S. M. \& Mayer, M. P. Dynamics of the regulation of Hsp90 by the co-chaperone Sti1. EMBO J 31, 1518-1528, doi:10.1038/emboj.2012.37 (2012). molecular chaperone revealed in the cryo-EM structure of the human Hsp90:Hop complex. Mol Cell 42, 771-781, doi:10.1016/j.molcel.2011.04.023 (2011). Sti1/Hop in Regulating the Hsp90 Chaperone Cycle. Genetics 209, 1139-1154, doi:10.1534/genetics.118.301178 (2018).

709

Verba, K. A. et al. Atomic structure of Hsp90-Cdc37-Cdk4 reveals that Hsp90 traps and stabilizes an unfolded kinase. Science 352, 1542-1547, doi:10.1126/science.aaf5023 (2016).

Bohen, S. P. Hsp90 mutants disrupt glucocorticoid receptor ligand binding and destabilize aporeceptor complexes. J Biol Chem 270, 29433-29438, doi:10.1074/jbc.270.49.29433 (1995).

Liu, Y. et al. Cryo-EM structures reveal a multistep mechanism of Hsp90 activation by co-chaperone Aha1. bioRxiv (2020).

Rutz, D. A. et al. A switch point in the molecular chaperone Hsp90 responding to client interaction. Nat Commun 9, 1472, doi:10.1038/s41467-018-03946-x (2018). 
71070 Suren, T. et al. Single-molecule force spectroscopy reveals folding steps associated with hormone binding and activation of the glucocorticoid receptor. Proc Natl Acad Sci U S A 115, 11688-11693, doi:10.1073/pnas.1807618115

713 (2018).

71471 Chin, J. W., Martin, A. B., King, D. S., Wang, L. \& Schultz, P. G. Addition of a photocrosslinking amino acid to the genetic code of Escherichiacoli. Proc Natl Acad Sci U S A 99, 11020-11024, doi:10.1073/pnas.172226299 (2002).

Schorb, M., Haberbosch, I., Hagen, W. J. H., Schwab, Y. \& Mastronarde, D. N. Software tools for automated transmission electron microscopy. Nat Methods 16, 471-477, doi:10.1038/s41592-019-0396-9 (2019).

Zheng, S. Q. et al. MotionCor2: anisotropic correction of beam-induced motion for improved cryo-electron microscopy. Nat Methods 14, 331-332, doi:10.1038/nmeth.4193 (2017).

723

74 Rohou, A. \& Grigorieff, N. CTFFIND4: Fast and accurate defocus estimation from

724

725 electron micrographs. J Struct Biol 192, 216-221, doi:10.1016/j.jsb.2015.08.008 (2015). structure of the stress-inducible human heat shock protein 70 substrate-binding domain in complex with peptide substrate. PLoS One 9, e103518, doi:10.1371/journal.pone.0103518 (2014). homology detection and structure prediction. Nucleic Acids Res 33, W244-248, doi:10.1093/nar/gki408 (2005).

Song, Y. et al. High-resolution comparative modeling with RosettaCM. Structure 21, 1735-1742, doi:10.1016/j.str.2013.08.005 (2013).

Schneider, M. et al. BiPPred: Combined sequence- and structure-based prediction of peptide binding to the Hsp70 chaperone BiP. Proteins 84, 13901407, doi:10.1002/prot.25084 (2016).

Gutierres, M. B. B., Bonorino, C. B. C. \& Rigo, M. M. ChaperISM: improved chaperone binding prediction using position-independent scoring matrices. Bioinformatics 36, 735-741, doi:10.1093/bioinformatics/btz670 (2020).

80 Zahn, M. et al. Structural studies on the forward and reverse binding modes of peptides to the chaperone DnaK. J Mol Biol 425, 2463-2479, doi:10.1016/j.jmb.2013.03.041 (2013).

81 DiMaio, F., Zhang, J., Chiu, W. \& Baker, D. Cryo-EM model validation using independent map reconstructions. Protein Sci 22, 865-868, doi:10.1002/pro.2267 (2013). 
74782 Wang, R. Y. et al. Automated structure refinement of macromolecular assemblies

748 from cryo-EM maps using Rosetta. Elife 5, doi:10.7554/eLife.17219 (2016).

74983 Bledsoe, R. K. et al. Crystal structure of the glucocorticoid receptor ligand binding 750 domain reveals a novel mode of receptor dimerization and coactivator

751 recognition. Cell 110, 93-105, doi:10.1016/s0092-8674(02)00817-6 (2002).

75284 Johnson, J. L., Halas, A. \& Flom, G. Nucleotide-Dependent Interaction of

753 Saccharomyces cerevisiae Hsp90 with the Cochaperone Proteins Sti1, Cpr6, and

$754 \quad$ Sba1. Mol Cell Biol 27, 768-776 (2007).

75585 Dey, B., Caplan, A. J. \& Boschelli, F. The Ydj1 molecular chaperone facilitates

756 formation of active p60v-src in yeast. Mol Biol Cell 7, 91-100 (1996). 
a

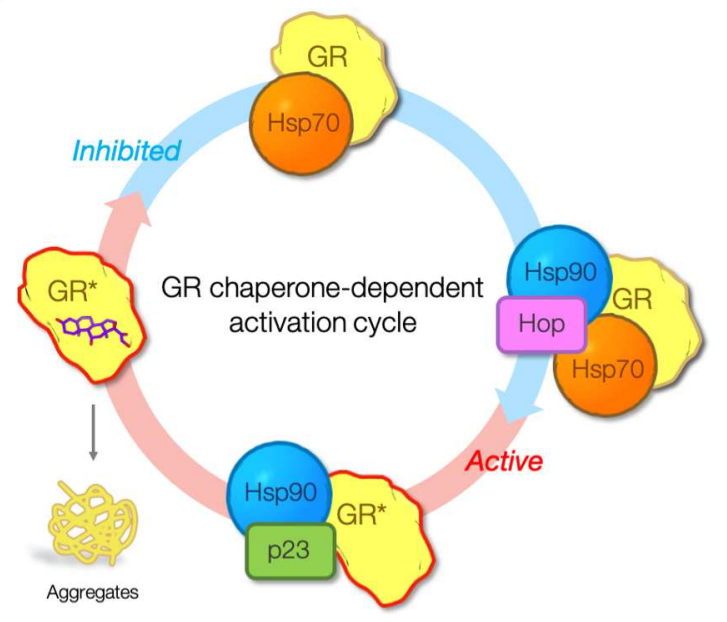

d

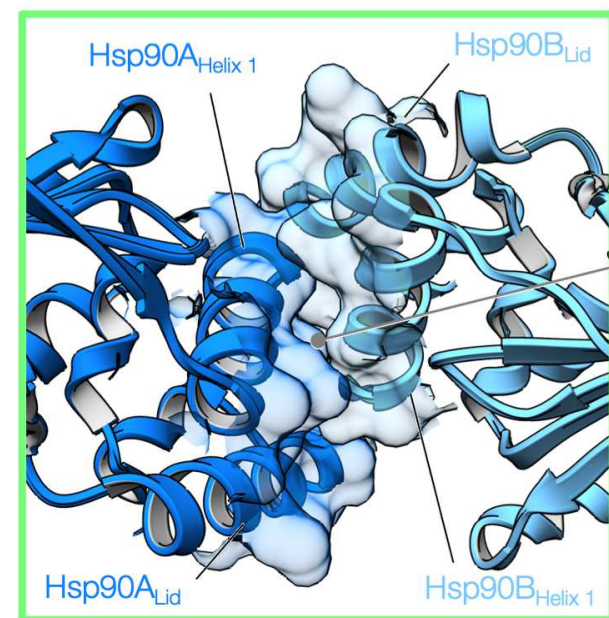

b

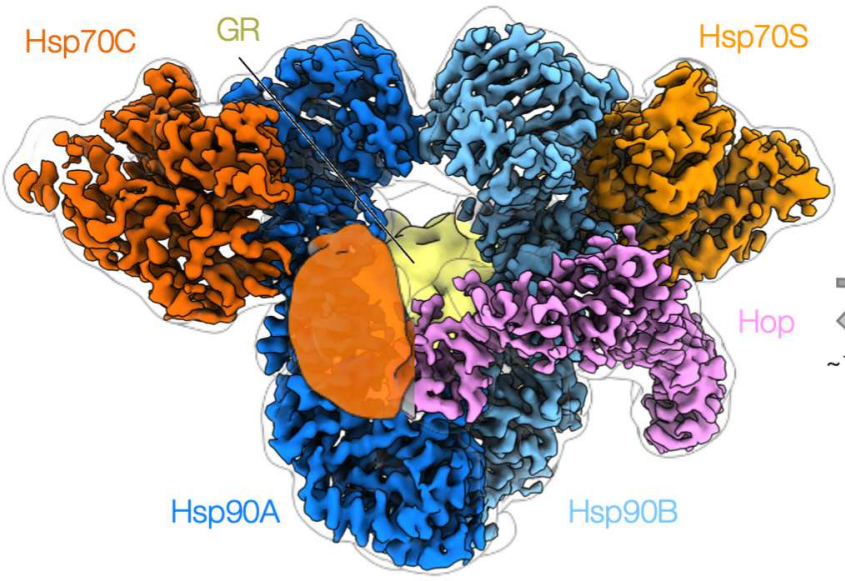

$\mathbf{e}$

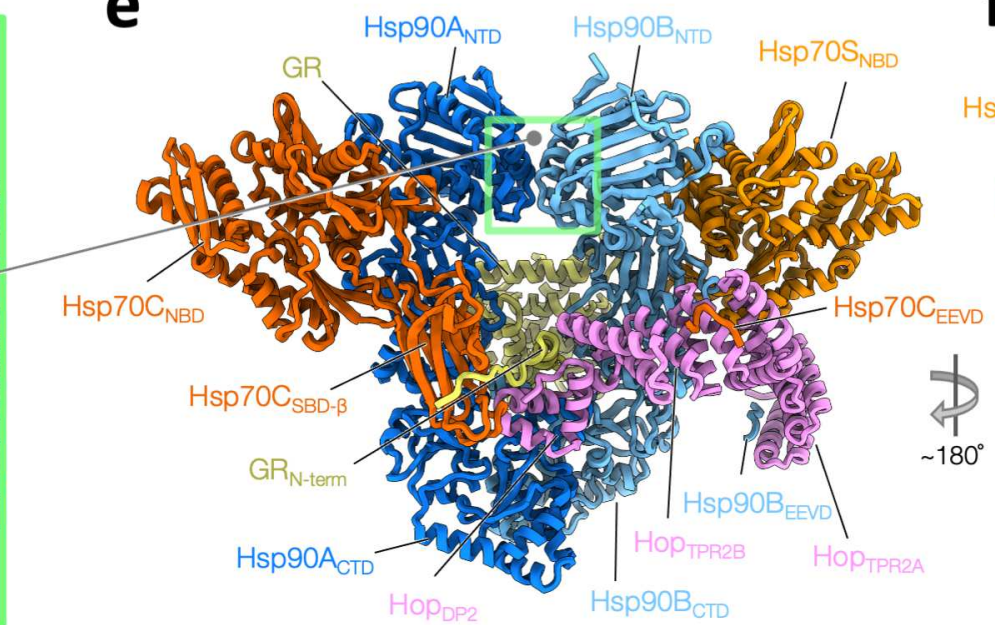

C

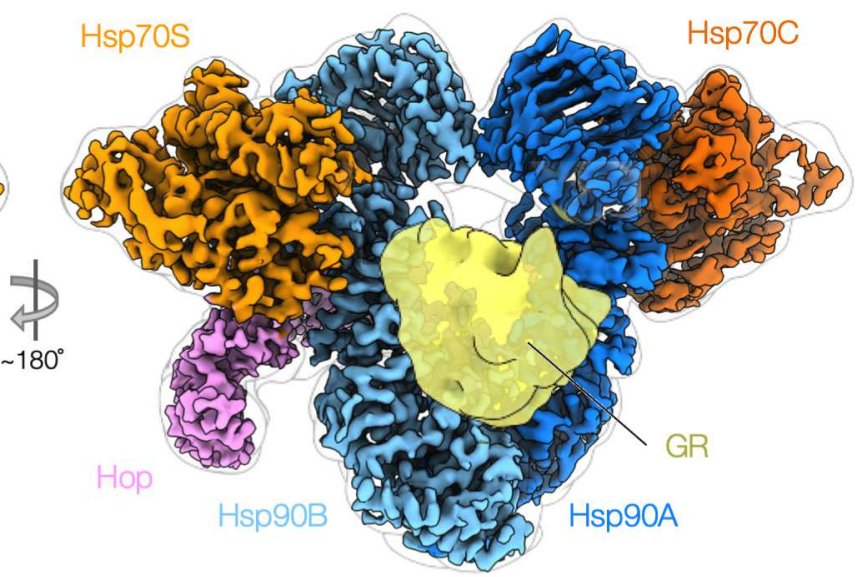

f

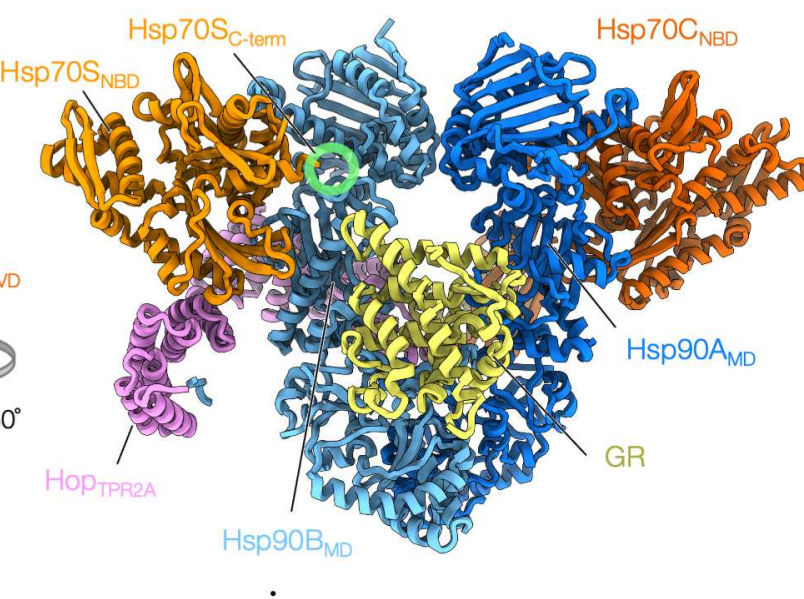

Fig. 1 | Overview of the GR-loading complex. a, GR activity is regulated by molecular chaperones in a constant cycle. Starting from active GR (left), which is aggregation-prone (lower left) in physiological conditions, Hsp70 protects and inhibits GR (top). Recruited by Hop, Hsp70 loads GR to Hsp90, forming the loading complex, in which GR remains inactive. Upon ATP hydrolysis on Hsp90, GR is reactivated in the maturation complex of GR:Hsp90:p23 (bottom) and is thereafter released to continue the cycle. b,c, Front (b) and back (c) views of a composite cryo-EM map of the GR:Hsp90:Hsp70:Hop complex. Densities of Hsp70C ${ }_{\mathrm{SBD}}$ and the globular C-terminal GR (yellow) are taken from the low-pass-filtered map of the highresolution reconstruction of the full complex. The subunit color code is used throughout. d, Close-up view of the novel dimerization interface of the symmetric Hsp90 dimer with residues at the interface in surface representation. The interface is composed of two molecular switches of Hsp90, the first helix and the lid motif. e,f, Corresponding views of the atomic model of the GR-loading complex. Green circle indicates the C-terminus of Hsp70S. 


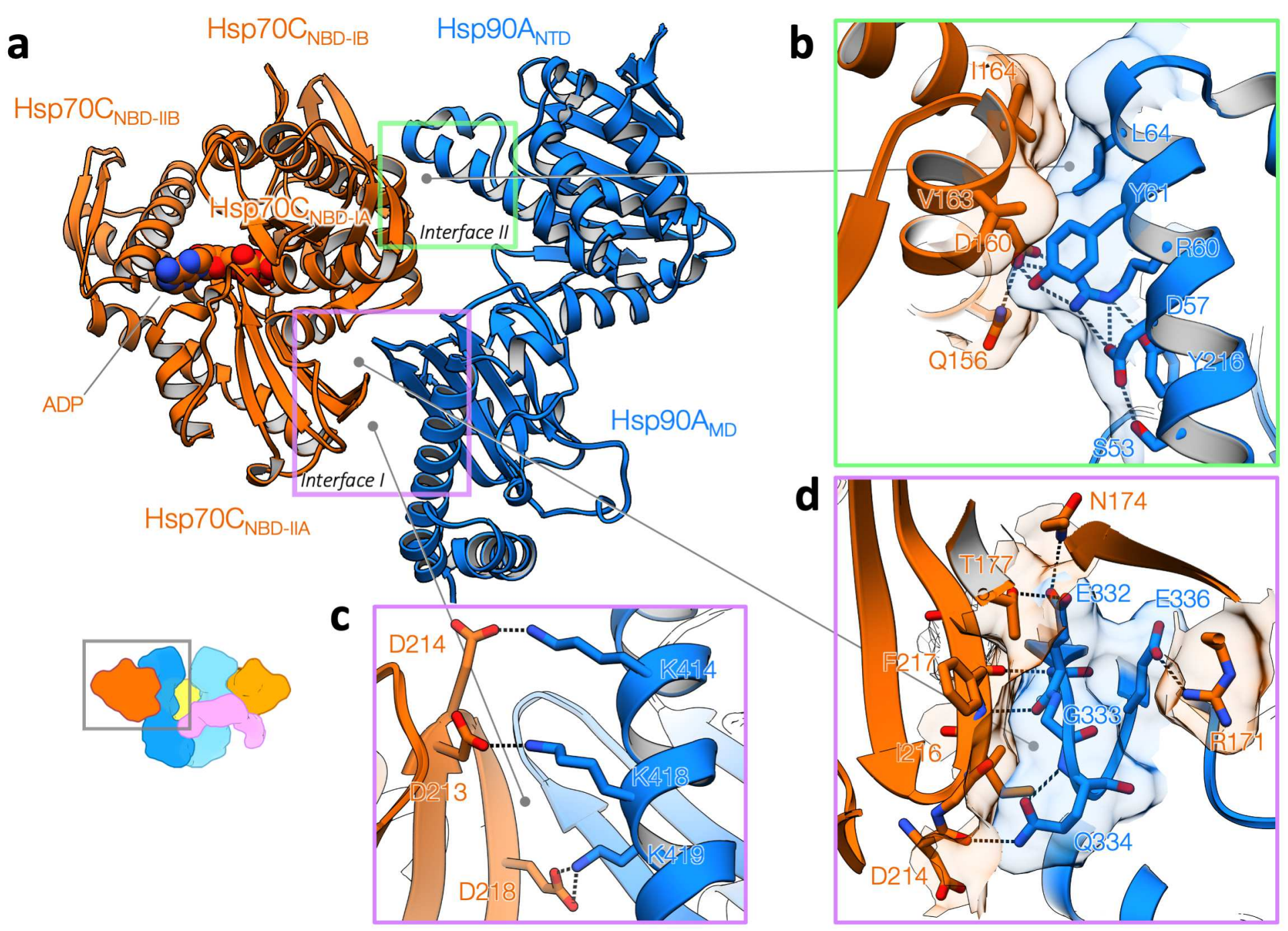

Fig. 2 | Molecular basis of Hsp90:Hsp70 interactions. a, Hsp90:Hsp70 interactions without the aid of Hop (Hsp90A:Hsp70C). In Interface I (purple rectangle), Hsp90 uses the outer edge of the Hsp90A $\mathrm{AD}_{\mathrm{MD}} \beta$-sheet to insert into a cleft formed by Hsp70 $\mathrm{NBD}_{\mathrm{N}-\mathrm{A}}$ and Hsp70 $\mathrm{NBD}_{\mathrm{NBA}}$ subdomains. The cleft is where the Hsp70 interdomain linker binds and serves as the allosteric center for Hsp70 NBD to regulate client binding in the Hsp70 SBD. In Interface II (green rectangle), the two ATPase domains Hsp90 and Hsp70 directly interact with each other. b, Close-up view of Interface II. Transparent surface and stick representations are shown for residues involved in the interactions. Dashed lines depict the network of polar interactions involved. c, Interface I is featured with conserved salt bridges (dashed lines). d, Detailed view of Interface II. A $\beta$-strand pairing (dashed lines) between the backbone atoms of Hsp90 ${ }^{\mathrm{E} 332}$ and Hsp70F217, in which Hsp90G333 is closely packed with Hsp70F217. 
a
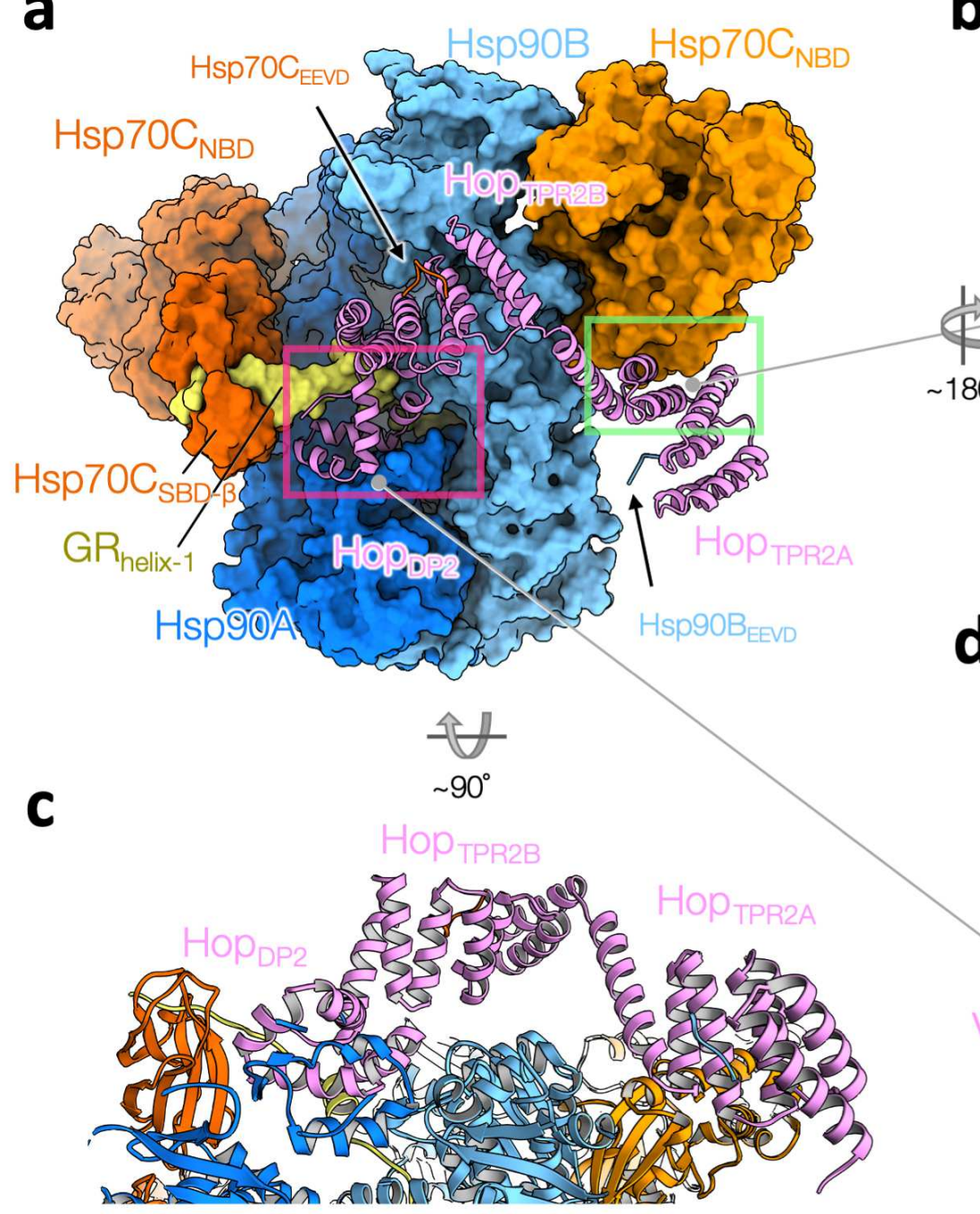

b
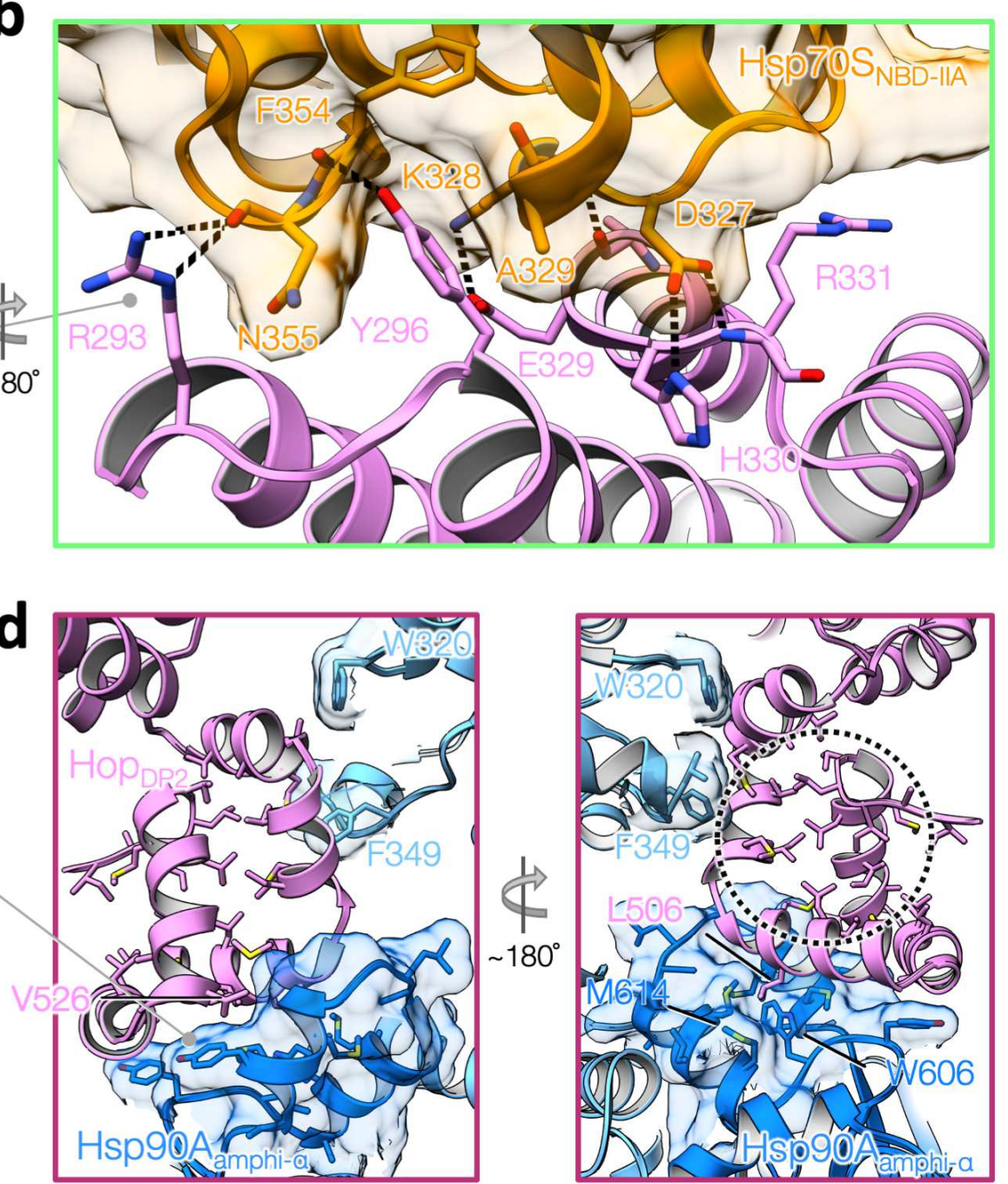

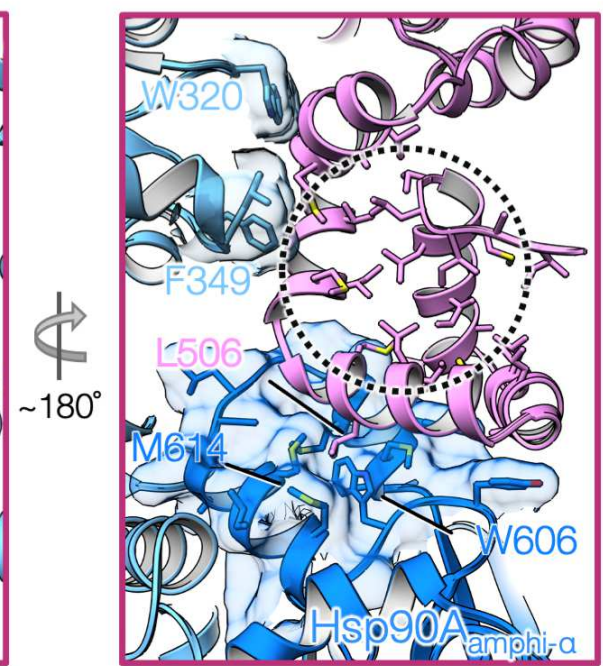

Fig. 3 | Hop interacts intimately with all components in the loading complex. a, Hop, shown in ribbon representation, uses its three C-terminal domains (Hop TPR2A, $_{\text {, Hop }}$ TPR2B $_{\text {and }}$ and $\mathrm{Hop}_{\mathrm{DP} 2}$ ) to interact with Hsp90/Hsp70 beyond the EEVD binding. All the other components are shown in surface representation. The red rectangle highlights that Hop $\mathrm{DPP}_{2}$ interacts with Hsp90A (dark blue), Hsp90B (light blue), Hsp70C $\mathrm{SBD}_{\mathrm{SD}}$ (dark orange) and a portion

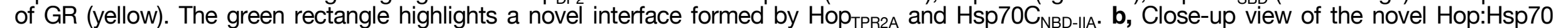
interface with a 180-degree rotation from (a). Hop ${ }^{\mathrm{Y} 296}$ inserts into a cavity on Hsp70 (transparent surface representation), forming a hydrogen bond with the Hsp70F354 backbone atom. The interface also features many polar interactions depicted with dashed lines. c, A 90-degree rotation from the bottom view in (a) with ribbon model. No major interaction is observed between Hop TPR2B and the loading complex. d, Left, Hop DP2 $_{2}$ uses surfaceexposed hydrophobic residues, shown in sticks, to interact with Hsp90's client-binding motifs (shown in transparent surface and with hydrophobic residues in sticks) - the amphipathic helical hairpin from Hsp90A ${ }_{\text {CTD }}$ (dark blue) and Hsp90Bw320,F349 (light blue). Right, a $\sim 180$-degree view from the left, Hop ${ }_{D P 2}$ adopts a hand-like a-helical structure. The core of Hop circle) of the "hand". Note the GR-binding to Hop ${ }_{\mathrm{DP} 2}$ is not shown here. 

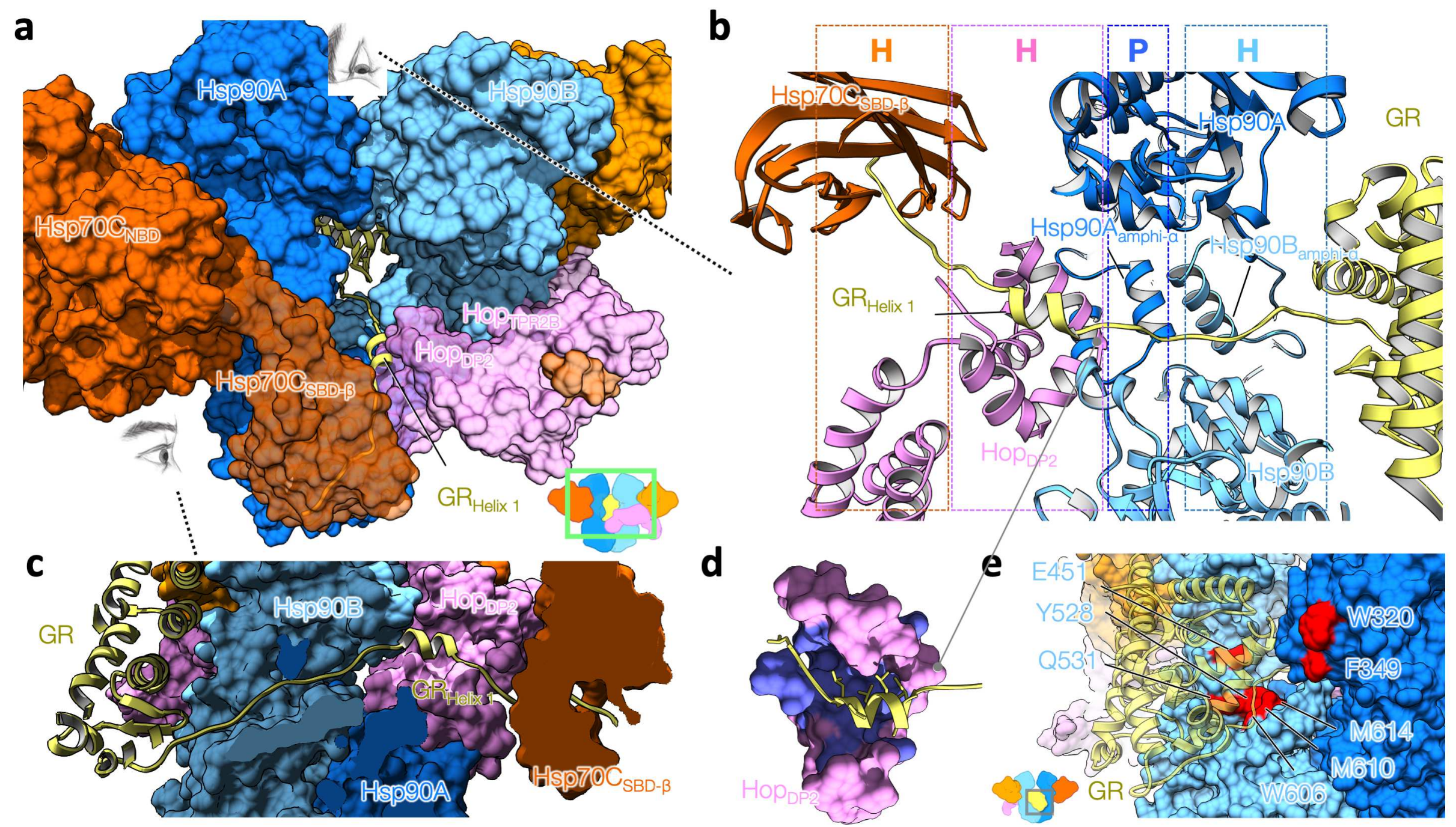

Fig. 4 | Facilitated by Hop, GR is loaded onto Hsp90 by Hsp70. a, Close-up front view of the loading complex (shown in surface representation). GR, shown in the ribbon model, is partially unfolded, with the N-terminal residues simultaneously griped by Hsp70C and $\mathrm{Hop}_{\mathrm{DP}}$, and is threaded through the semi-closed lumen of Hsp90. The remaining GR is at the other side of the loading complex; the ribbon model shown for the major body of GR is a modeling result from docking the GR crystal structure to the low-pass-filtered GR density. $\mathbf{b}$, Topto-bottom view of GR recognition via an extended client-binding pocket collectively formed by $\mathrm{Hsp}_{\mathrm{s}} 70 \mathrm{C}_{\mathrm{SBD}-\beta}$, Hop $\mathrm{HP}_{\mathrm{DP}}$, and $\mathrm{Hsp} 90 \mathrm{~A} / \mathrm{B}_{\mathrm{amphi}-\mathrm{s}} \mathrm{S}$ in ribbon representation. The N-terminal residues of GR (residues 517-533), which form a strand-helix-strand motif (yellow), are captured in the loading complex. The molecular properties provided by the individual binding pockets are color coded and labelled on the top panel $(\mathrm{H}$ and $\mathrm{P}$ denote hydrophobic and polar interactions, respectively). c, Side view of the GR N-terminal motif captured by the loading complex. $\mathbf{d}$, Hop $\mathrm{DP}_{2}$, shown in surface representation, binds the LXXLL motif of GR Helix1, in which the hydrophobic residues of Hop ${ }_{\mathrm{DP} 2}$ are colored with purple and those of GR are shown in sticks. e, Residues on Hsp90 (surface representation) previously reported to be important for GR (transparent yellow ribbon) activation are highlighted in red. 
a
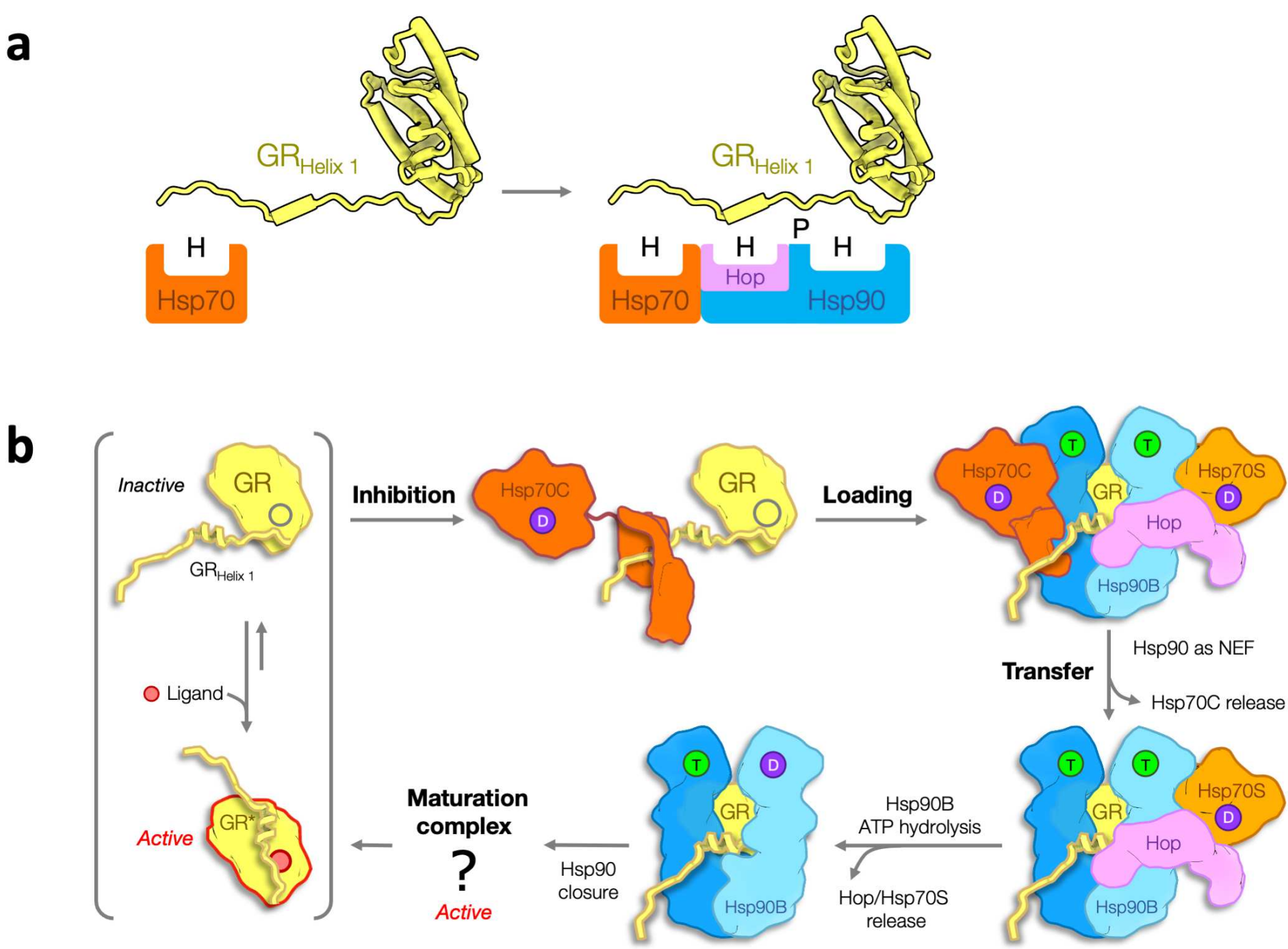

Fig. 5 | Schematic model of GR loading onto Hsp90 by Hsp70. a, The molecular principle of GR recognition at the client-loading step. Left, Hsp70 ${ }_{\text {SBD }}$ (orange) inhibits GR (yellow), providing mostly hydrophobic binding $(\mathrm{H})$. Right, the client-loading complex further stabilizes GR via an extended binding pocket assembled by Hsp70 ${ }_{\mathrm{SBD}}$ (orange), Hop $\mathrm{DP}_{2}$ (pink) and the lumen of Hsp90 dimer (blue), where stronger and more versatile molecular recognitions are provided for both hydrophobic $(\mathrm{H})$ and polar $(\mathrm{P})$ interactions. b, Molecular mechanism of GR transfer from Hsp70 to Hsp90 and the use of ATP hydrolysis on Hsp90. GR in physiological conditions is in an equilibrium of active and inactive states, in which the Helix 1 motif acts as a lid to stabilize ligand binding when attached. Hsp70C (dark orange) in its ADP state (D) binds GR's pre-Helix 1 strand, facilitating the following motif to detach and hence inhibit GR (top-middle). Facilitated by Hop ${ }_{\mathrm{DP} 2}$ recognizing the LXXLL motif on GR Helix 1, Hsp70C loads GR to Hsp90 (blue), forming the client-loading complex (top-right). Although the structure was determined in an Hsp90Apo state, we reason that in physiological conditions the high abundance of ATP would soon occupy Hsp90's ATP binding pockets (T). Hsp90's ATP binding and NEF activity facilitate Hsp70C release (bottom-right). The energy from the ATP hydrolysis (D) on Hsp90B (light blue) is used to release Hsp70S (light orange) and Hop (bottom-middle), followed by full closure of Hsp90. 
a

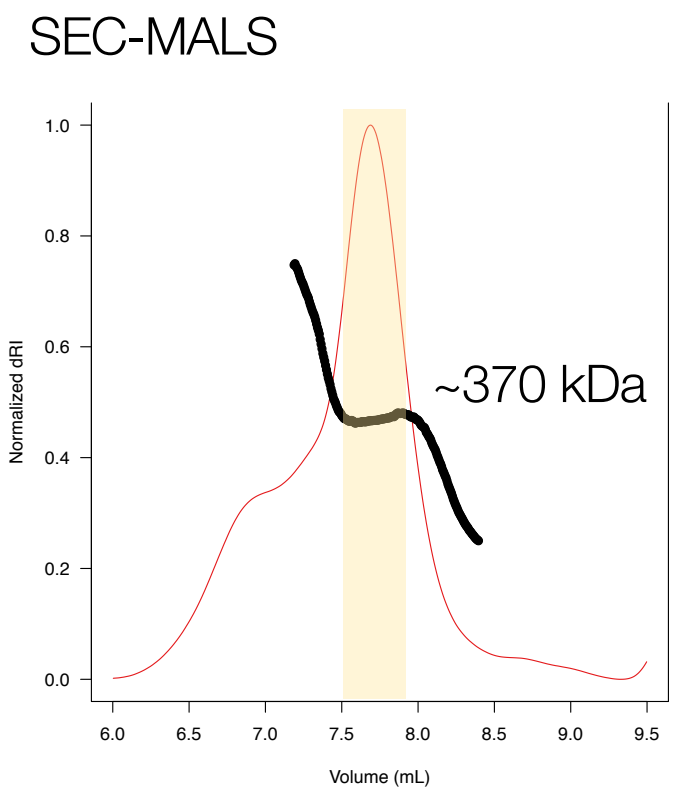

b

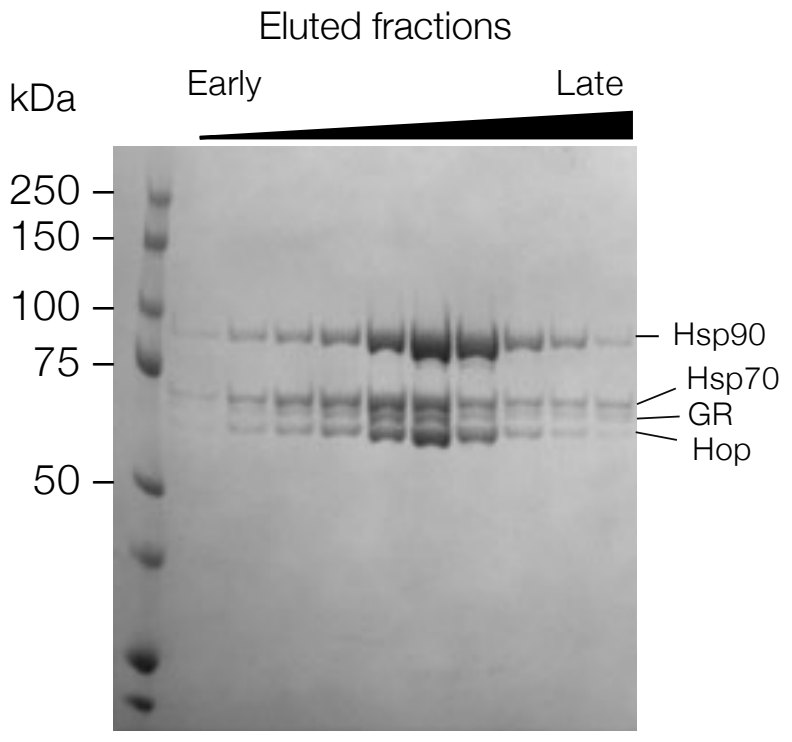

C

SDS-PAGE

cross-linked with glutaraldehyde

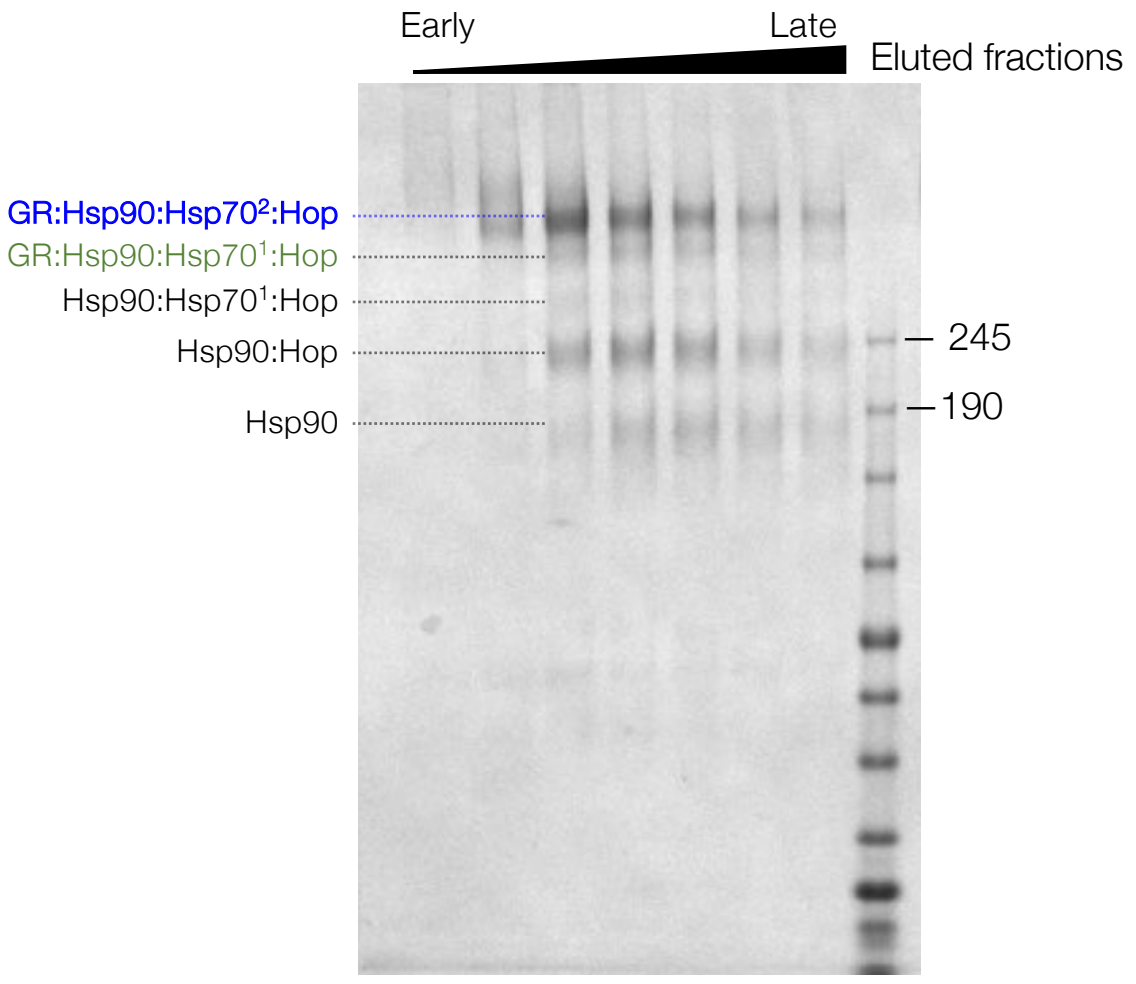

Extended Data Fig. 1 | In vitro reconstitution and purification of the GR-loading complex. a, Elution profile of gel filtration using SEC-MALS to confirm the homogeneity of the GR-loading complex. The apparent molecular weight of the eluent estimated by SEC-MALS is $\sim 370 \mathrm{kDa}$ although the two-Hsp70 client-loading complex is $\sim 440 \mathrm{kDa}$. The discrepancy may be a result of multiple species co-eluted. c, SDS-PAGE stained with Coomassie blue of the eluted fractions marked in (a). d, SDS-PAGE of the fractions treated with $0.02 \%(\mathrm{w} / \mathrm{v})$ glutaraldehyde crosslinking for 20 minutes at room temperature, followed by quenching with $20 \mathrm{mM}$ Tris buffer at $\mathrm{pH} 7.5$. 
a

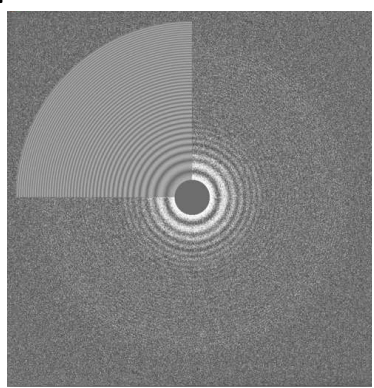

CTF estimation

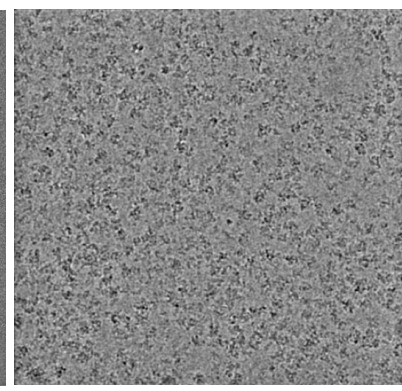

Micrograph

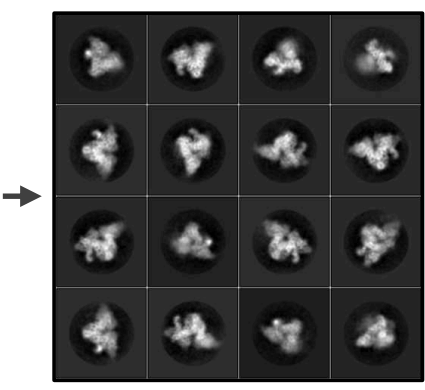

2D class averages
$60 \AA$ low-pass filter

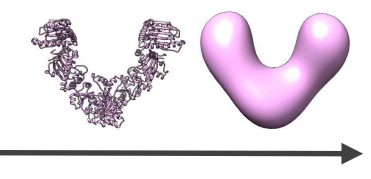

3D classification
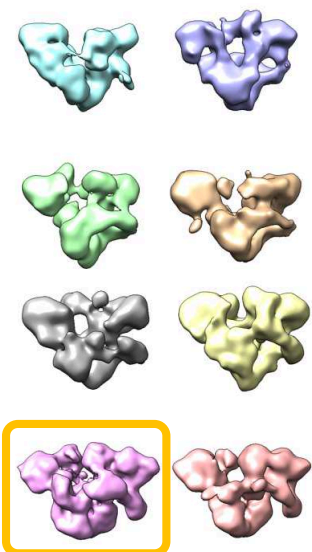

b

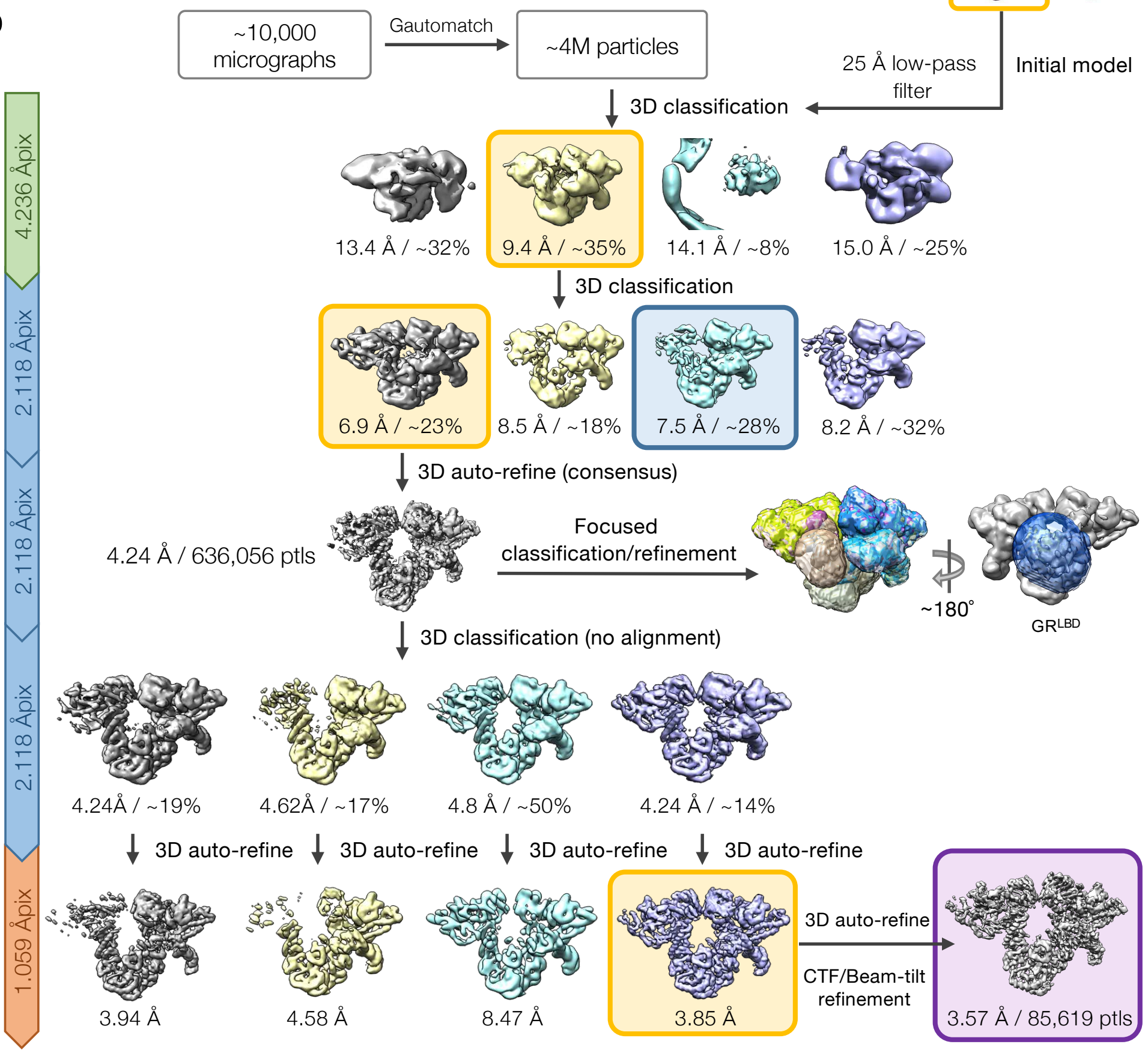

Extended Data Fig. 2 | Cryo-EM single-particle image processing pipeline of the GR-loading complex. a, Initial model generation for the GR-loading complex. The $60 \AA$ low-pass filtered initial model used to reconstruct the 3D model was adopted from the Hsp90 semi-open conformation structure from the Hsp90:Hop cryo-EM complex (Southworth \& Agard, 2011). b, Schematic workflow of the global cryo-EM map reconstruction. Yellow boxes indicate the selected class to move forward. Blue box indicates one-Hsp70 loading complex. Purple box indicates the final high-resolution global reconstruction. 
a

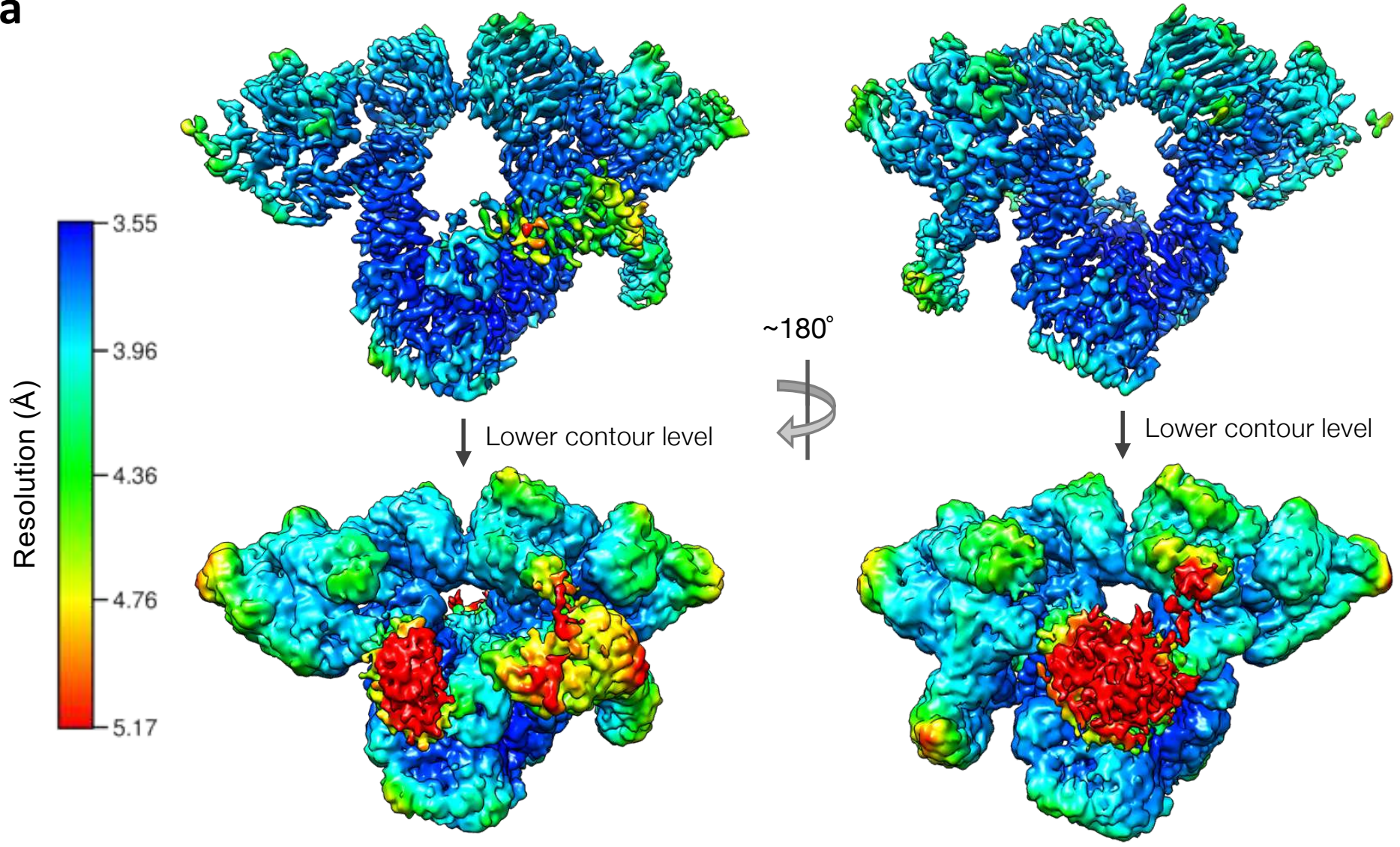

b

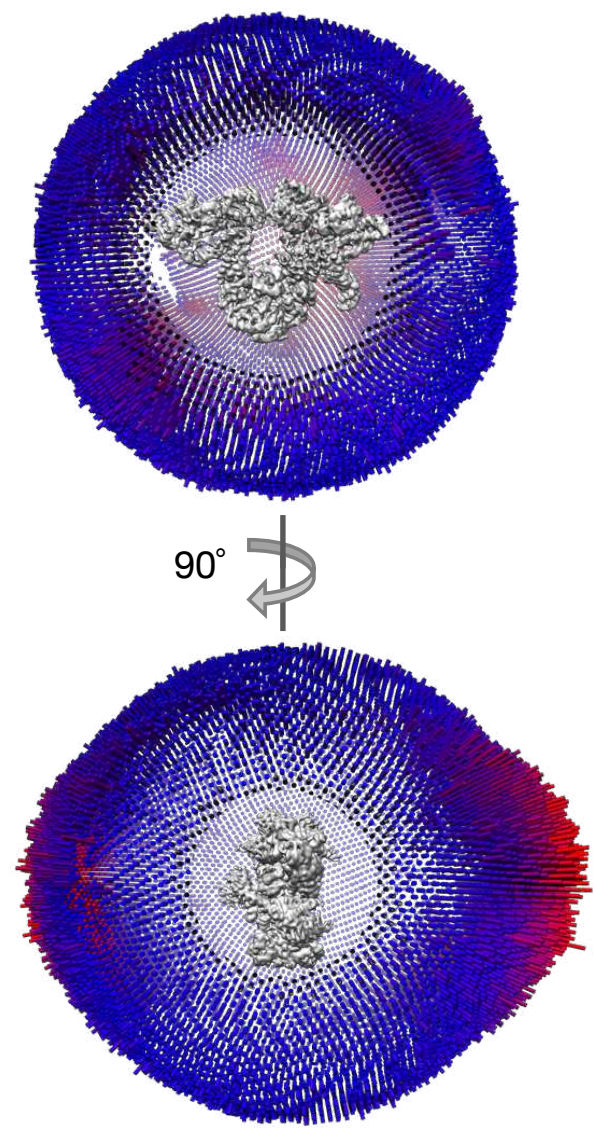

C

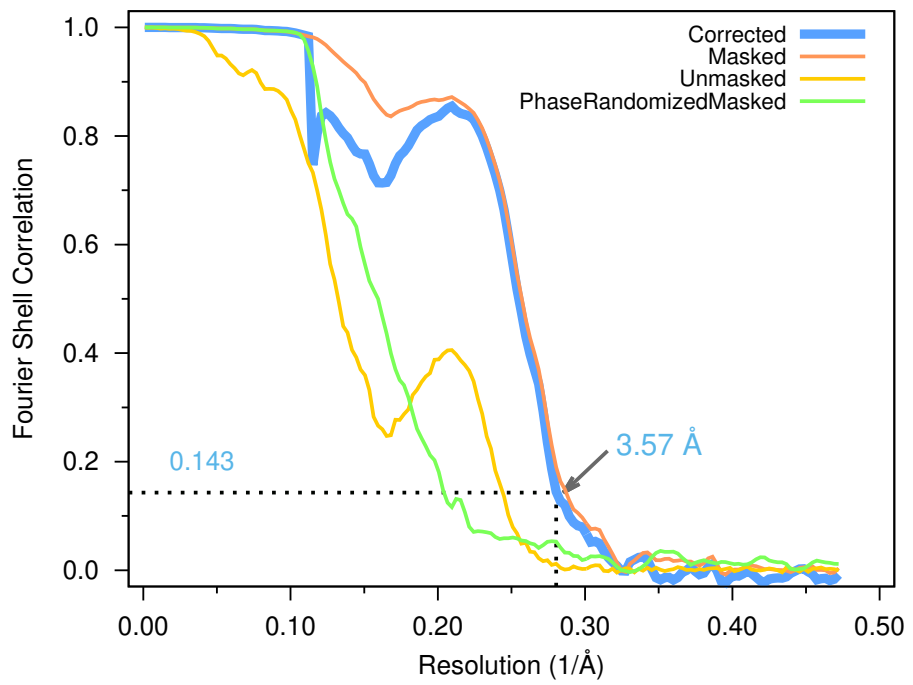

Extended Data Fig. 3 | Cryo-EM single-particle analysis of the GR-loading complex. a, Local resolution estimates for the GR-loading complex global reconstruction were calculated using RELION with front view (left) and back view (right). b, Euler angle distribution in the final reconstruction. Orthogonal views of the reconstruction are shown with front view (top) and side view (bottom) c, Gold Standard FSC for the global cryo-EM reconstruction (top). 


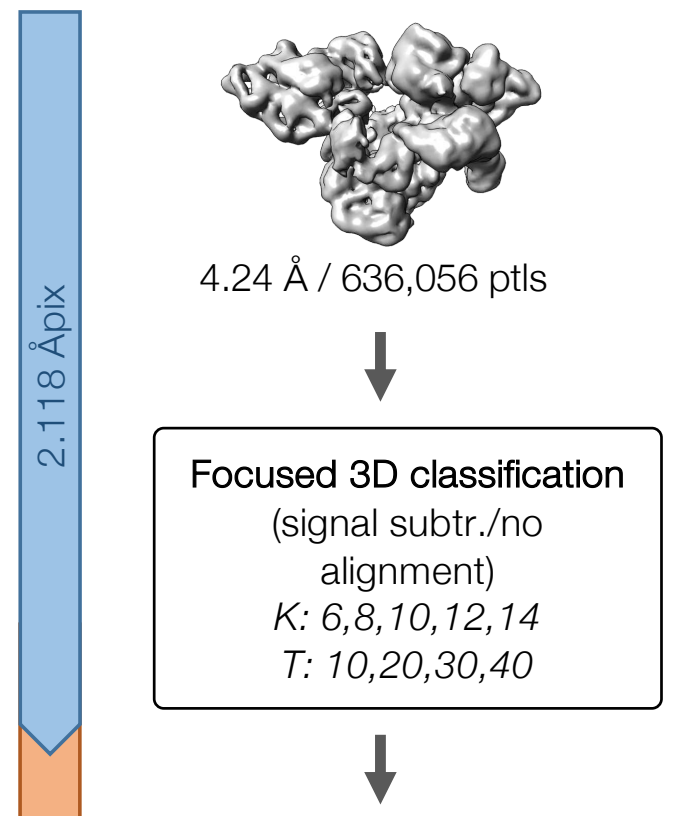

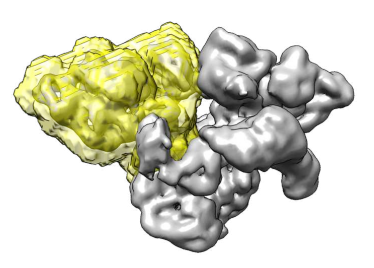

Hsp90ANTD-MD:Hsp70CNBD

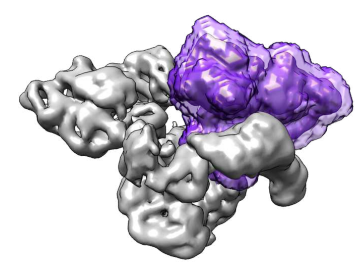

Hsp90BNTD-MD:Hsp70SNBD

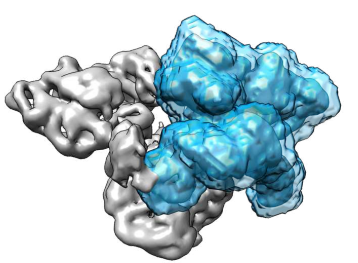

Hsp90B NTD-MD:Hsp70SNBD:HopTPR2A-TPR2B-DP2

\section{Focused 3D classification}

(signal subtr.)

$K: 2,3,4,5$

$T: 10,20,30,40$

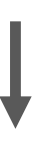

\section{D auto-refine \\ (masked)}

Hsp70SNBD:Hop TPR2A

\section{CTF/beam-tilt refinement}
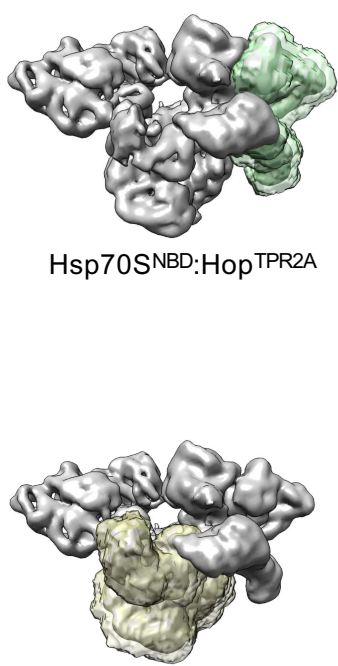

Hsp90AB CTD:Hsp70SSBD-B:Hop DP2:GR Helix 1
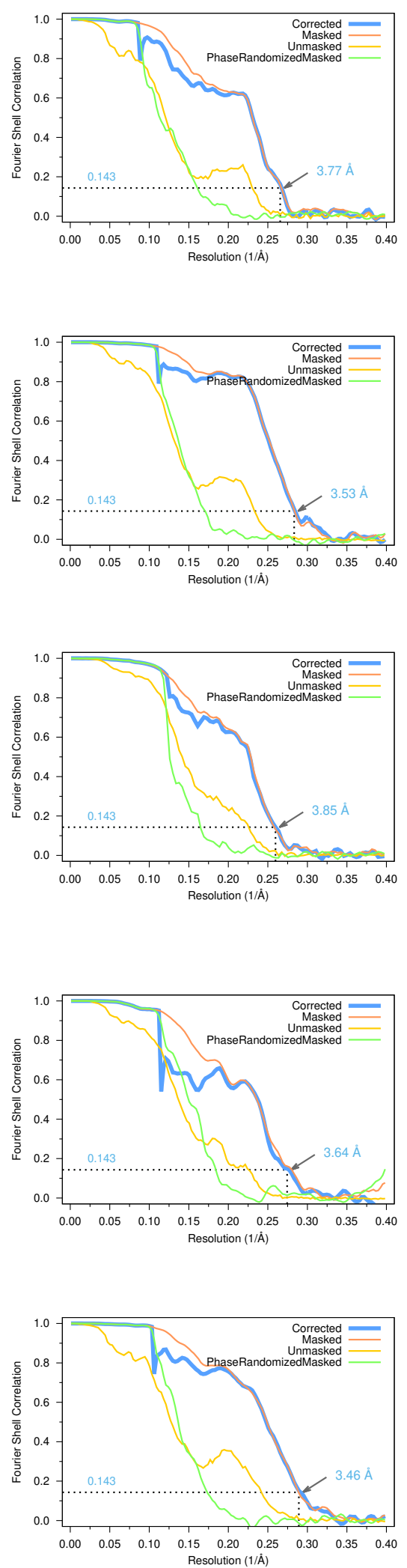

Extended Data Fig. 4 | Focused classification and refinement. a, Flow chart of focused classification/refinement using the signal subtraction approach. Final reconstructions for individual masked classifications/refinements were selected based on the resolution intercepted with the FSC 0.143 from 3D auto-refine. $\mathbf{b}$, Masks were created at various regions of the GR-loading complex (left) and its corresponding Gold Standard FSC (right) after 3D auto-refine. The nominal resolution for each reconstruction is labeled and indicated in the FSC plots. 
a
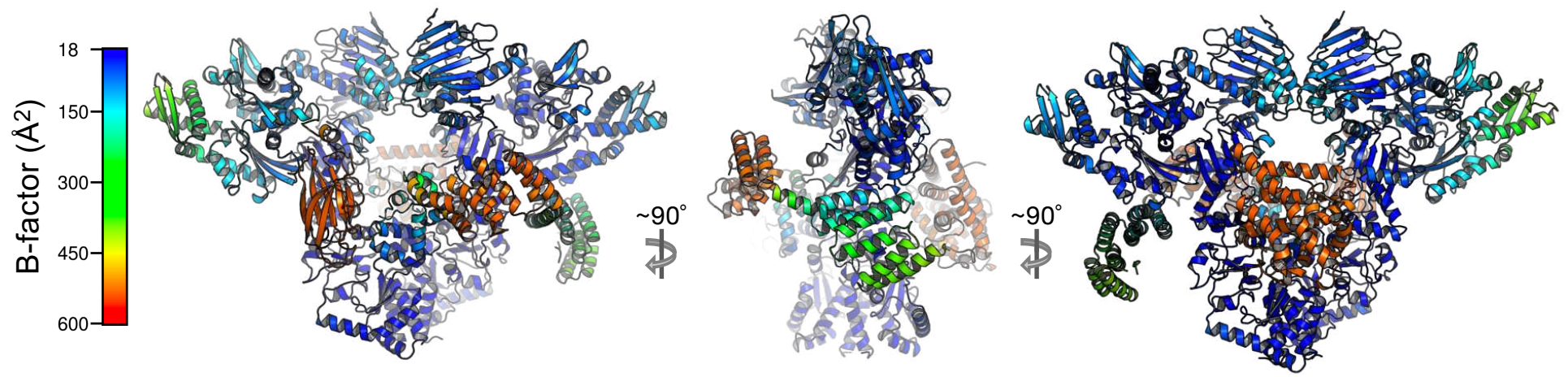

b

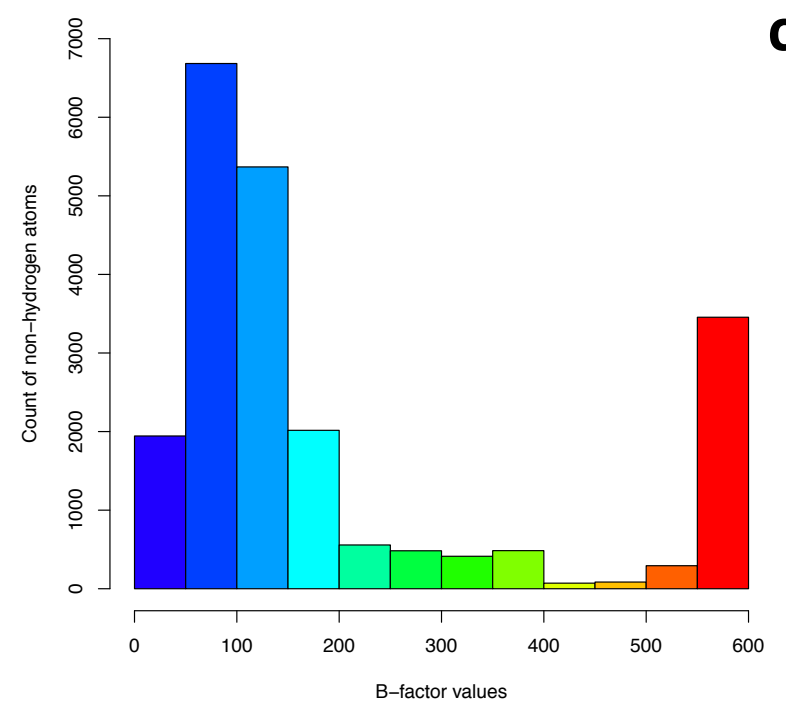

C

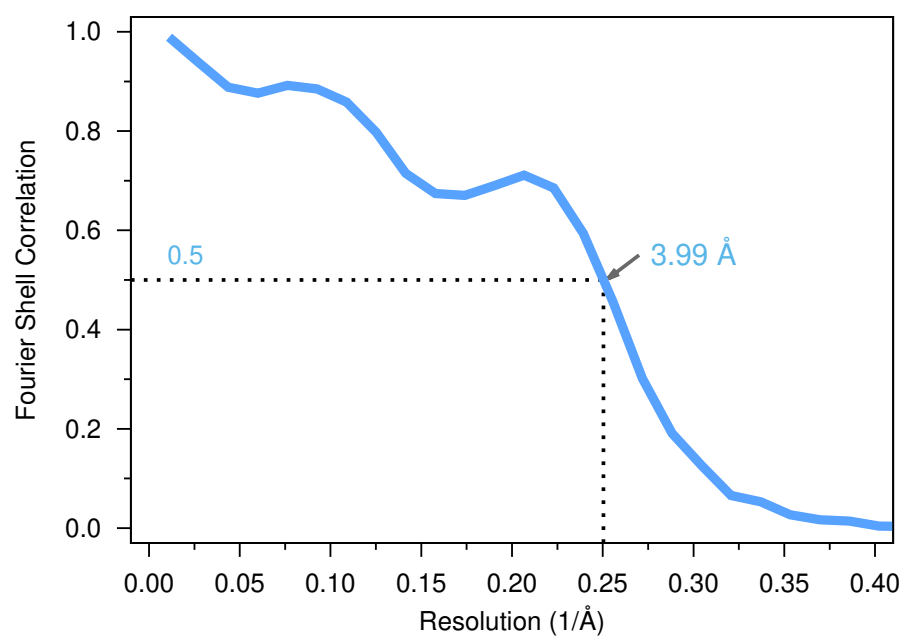

Extended Data Fig. 5 | Model B-factor refinement and model-map FSC. a, Atomic model with Bfactors refined with color key shown on the left. $\mathbf{b}$, Histogram of the B-factor values of all nonhydrogen atoms in the atomic model, colored by the same color key in (a). c, Model-map FSC. 
a
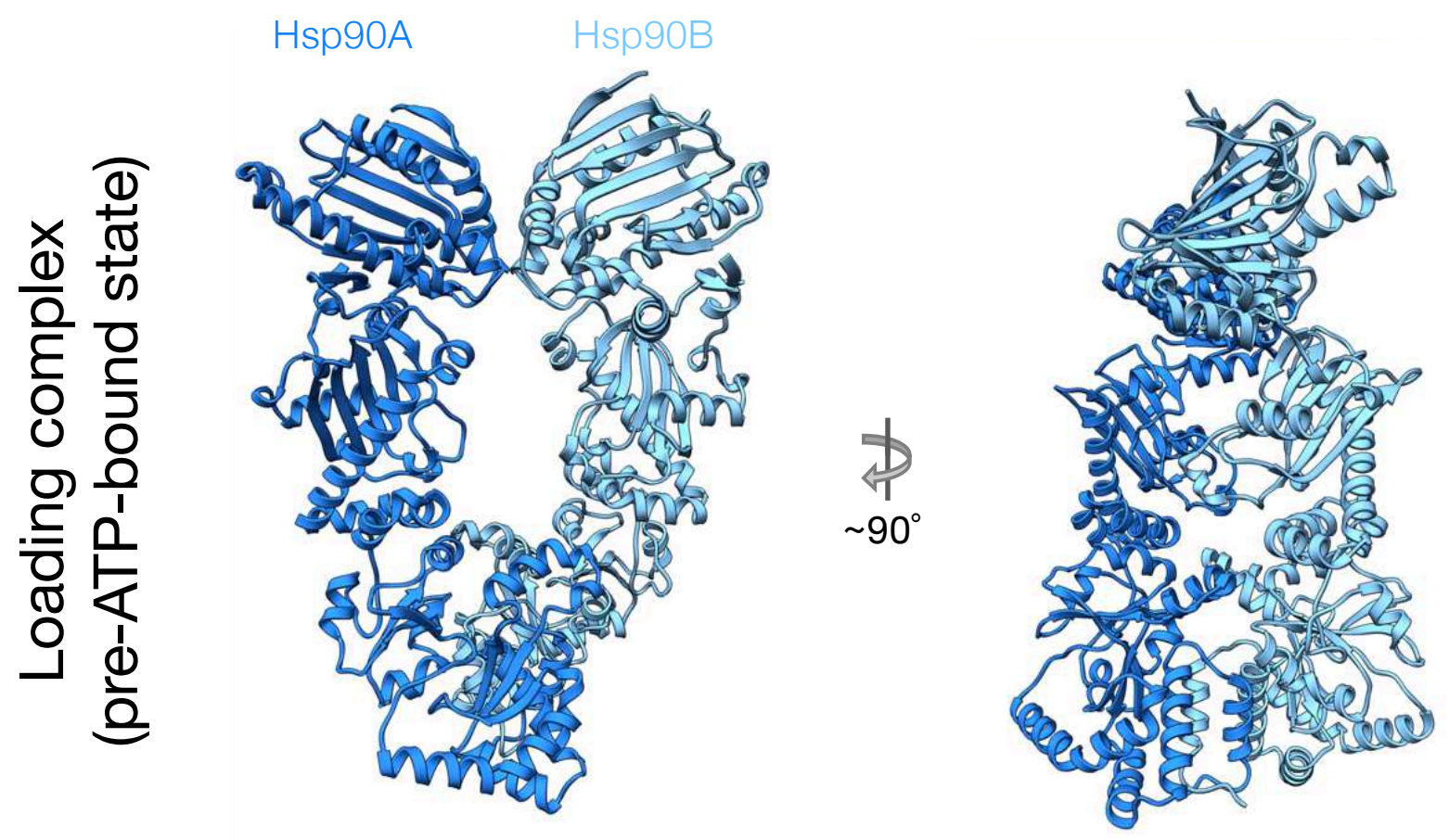

b
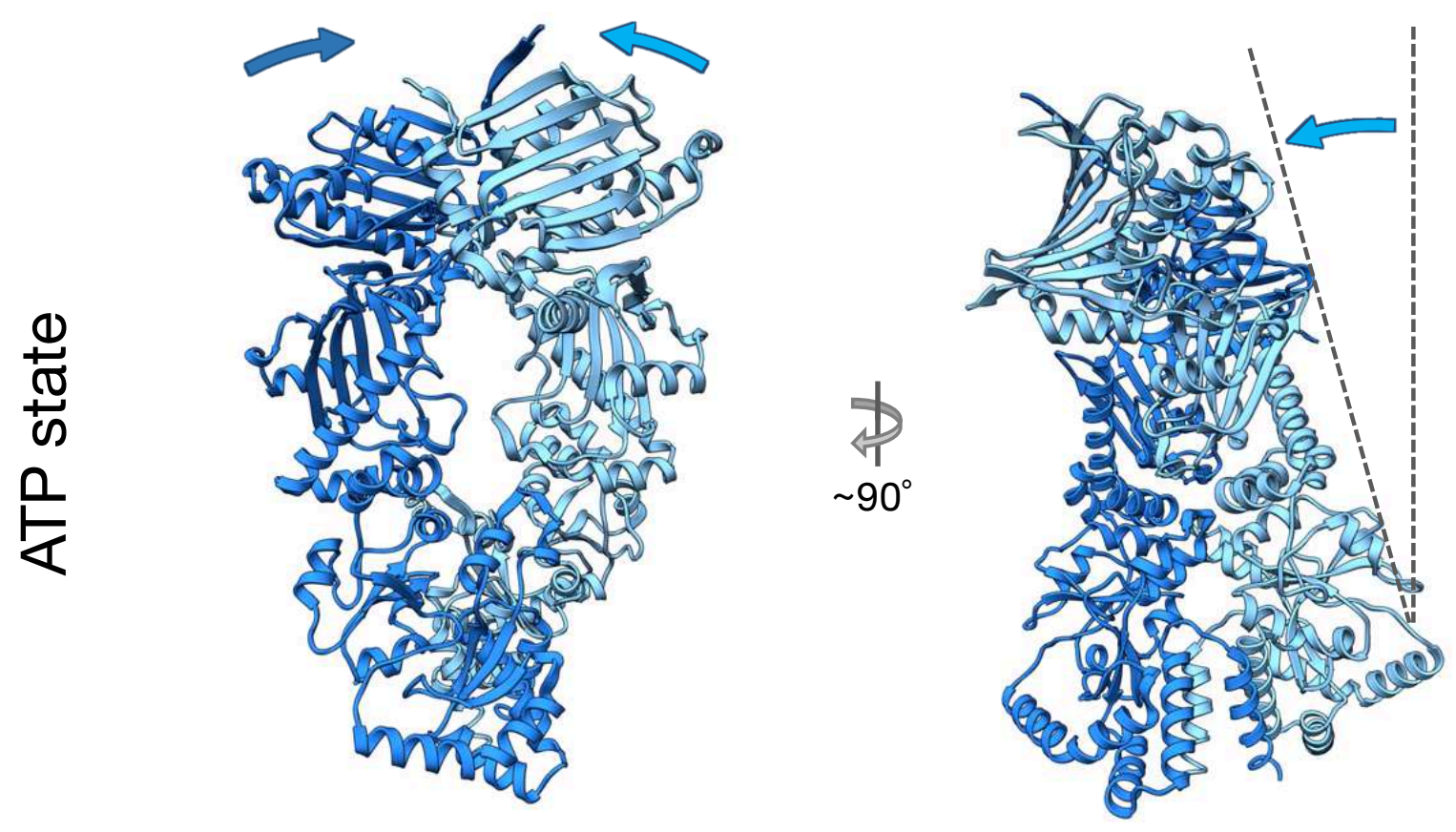

Extended Data Fig. 6 | The Hsp90 in the loading complex is one step away from the fully closed ATP state. a, Front (left) and side (right) views of the Hsp90 in the loading complex. b. Front and side views of Hsp90 in the ATP state (the Hsp90 in the GR-maturation complex). Arrows indicate displacements from the Hsp90 in the loading state, in which a large twisting motion is apparent from the side view. 
a
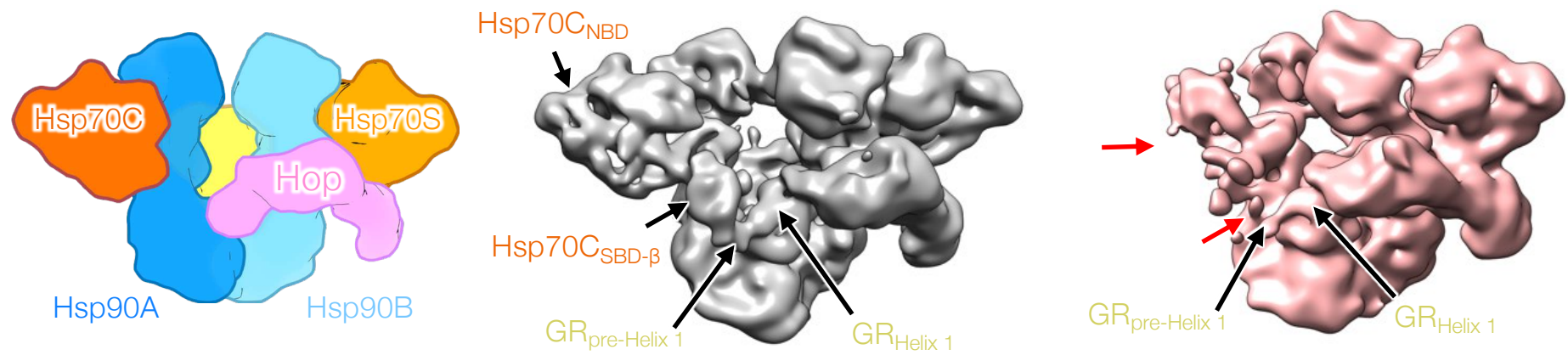

$$
\text { D) } 180^{\circ}
$$

$$
\text { D } 180^{\circ}
$$

$$
\text { D) } 180^{\circ}
$$

b
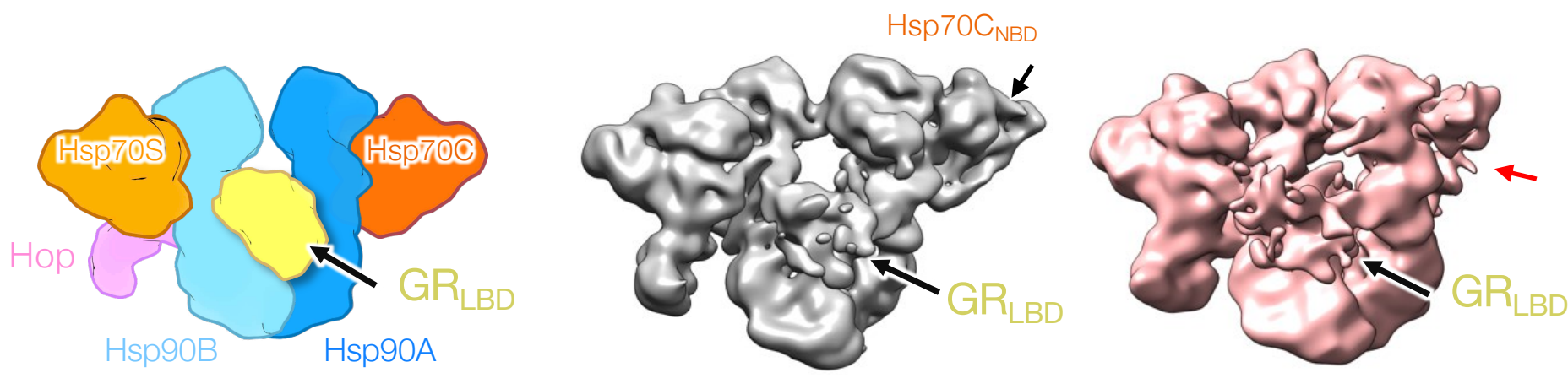

Extended Data Fig. 7 | One-Hsp70 GR-loading complex. a, Schematic model of the two-Hsp70 loading complex (left). Front views of cryo-EM maps of the two-Hsp70 (middle; gray color) and one-Hsp70 (right; salmon color) GR-loading complexes. Right, the one-Hsp70 reconstruction has lost density for Hsp70C NBD and Hsp70C SBD-a (red arrows); however, density for GR $\mathrm{R}_{\text {pre-Helix } 1}$ and $\mathrm{GR}_{\mathrm{Helix} 1}$ is in the same locations as it is in the two-Hsp70 GR-loading complex. b, 180 degree views from the top. 
a

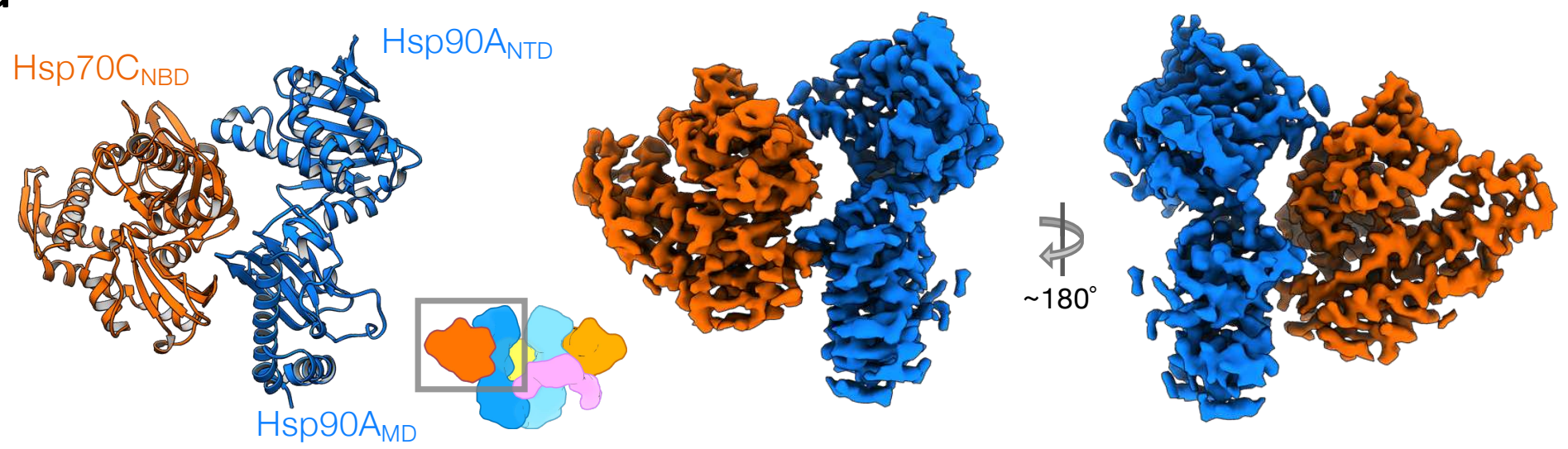

b

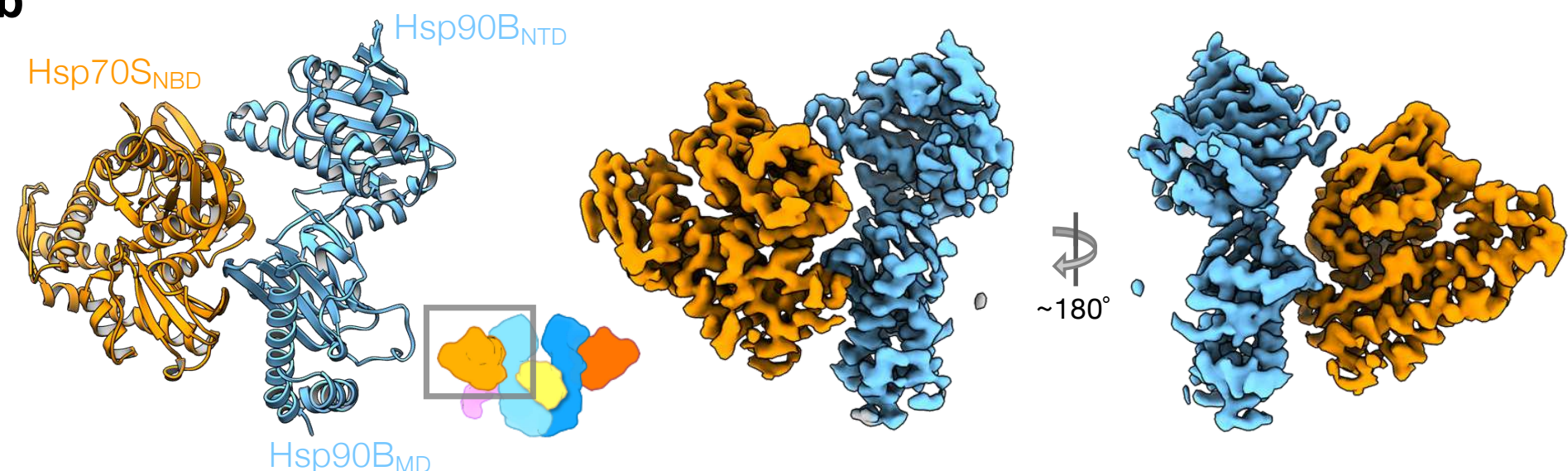

C
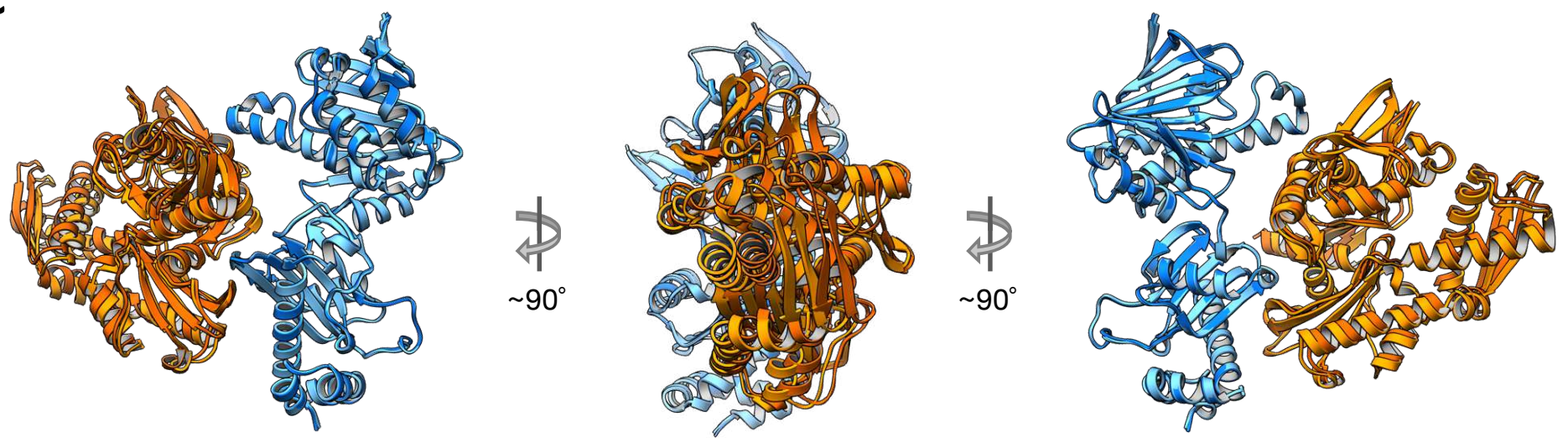

Extended Data Fig. 8 | Hsp90 and Hsp70 pairs in the GR-loading complex. a, Hsp90A and Hsp70C are shown in ribbon representation (left) colored in dark orange and dark blue, respectively. The corresponding focused map (middle and right) with a $3.77 \AA$ resolution. b. Hsp70B and Hsp70S are shown in ribbon representation (left) colored in orange and blue, respectively. The corresponding focused map (middle and right) with a $3.53 \AA$ resolution. c, Overlay of the Hsp90:Hsp70 pairs from (a) and (b) with Hsp90s aligned. 
a

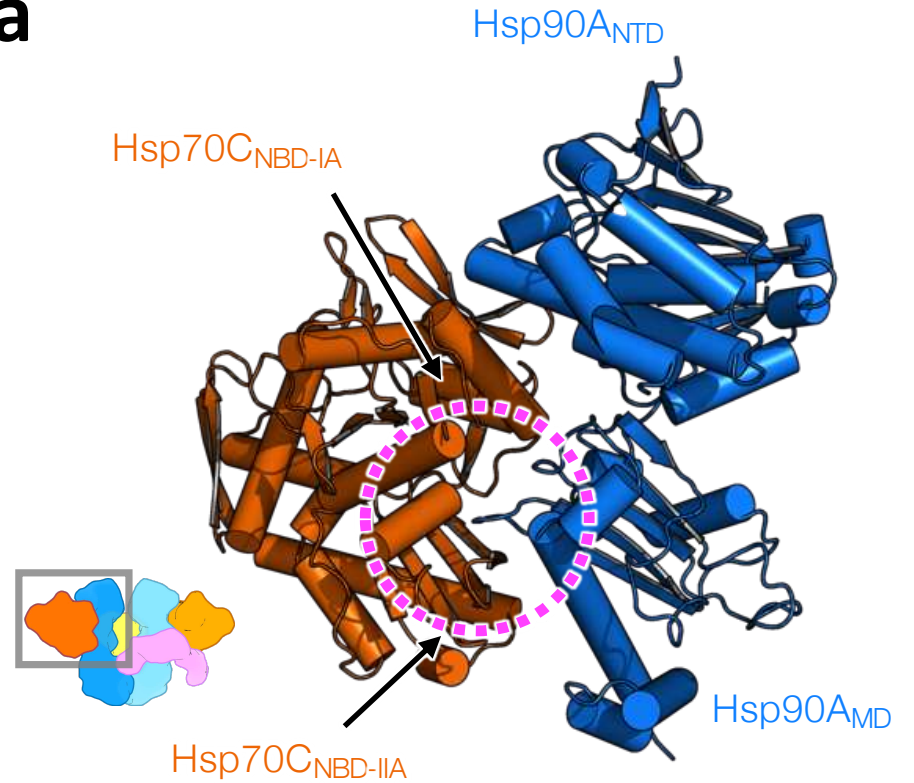

Hsp70C ${ }_{\text {NBD-IIA }}$

b

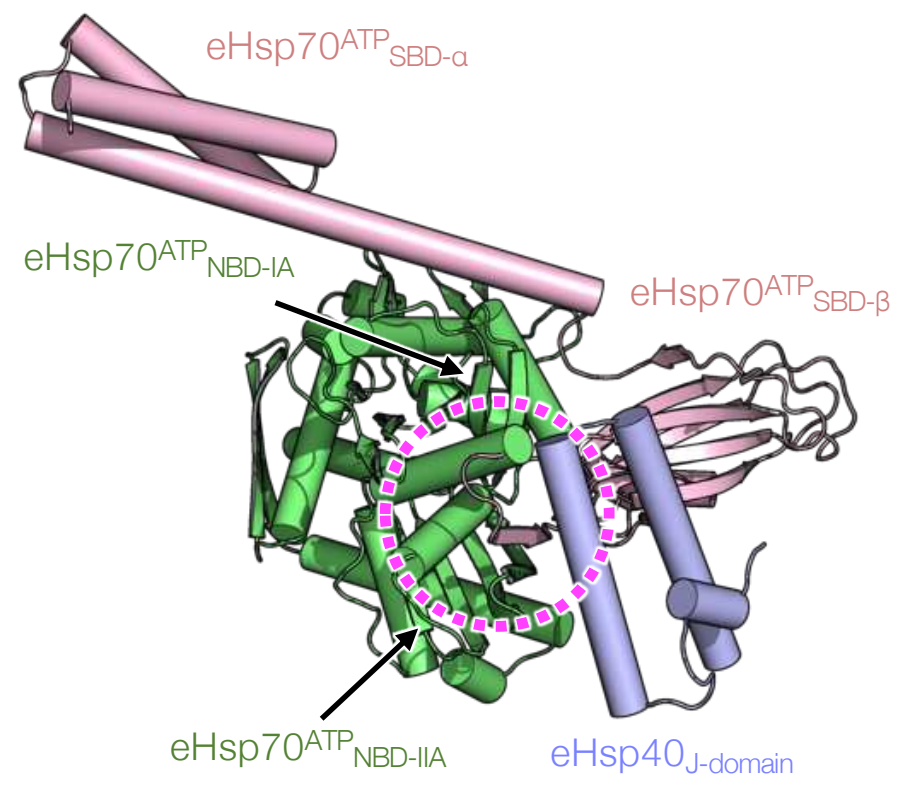

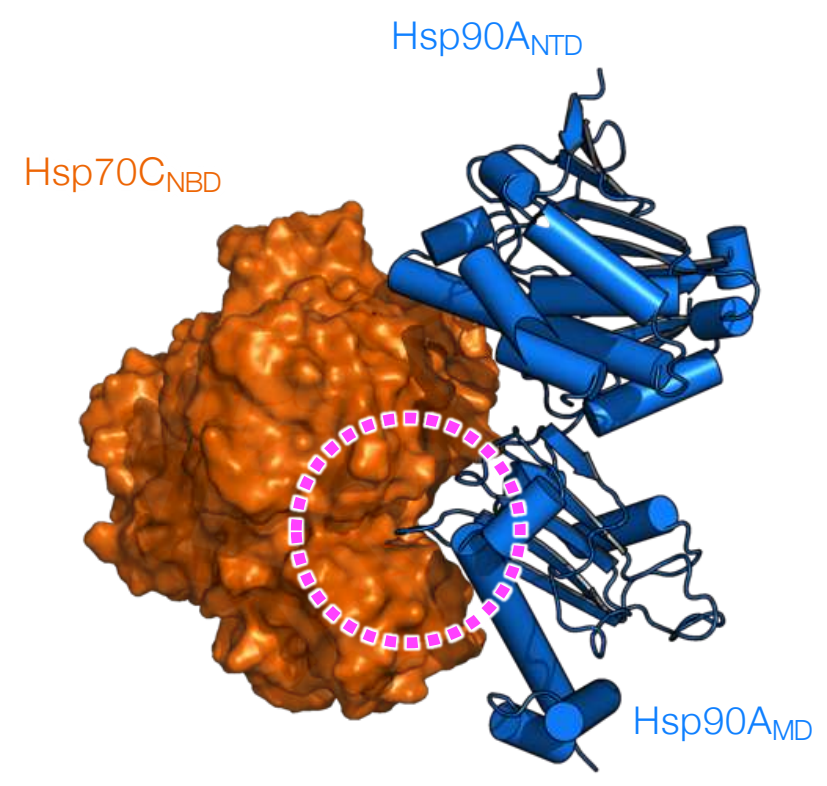

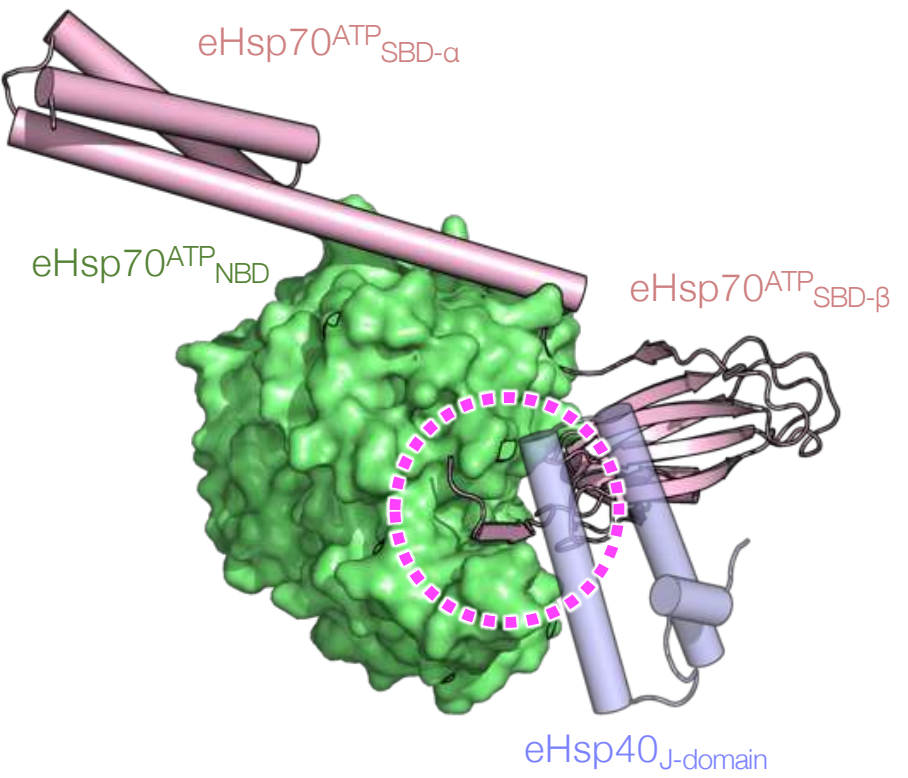

Extended Data Fig. 9 | The Hsp70 cleft, formed by Hsp70 ${ }_{\text {NBD-IA }}$ and Hsp70 ${ }_{\text {NBD-IIA, }}$ which Hsp90MD interacts with, is used by the interdomain linker in the Hsp70 ATP state and Hsp40's Jdomain. a, Cartoon (left) and surface (right) representation of Hsp90A:Hsp70C in the loading complex. The Hsp70 cleft is indicated by dashed circles. b, Cartoon (left) and surface (right) representation of the E. coli Hsp70 (eHsp70):J-protein complex in the ATP state (PDB ID: 5nro). The two subdomains of $\mathrm{Hsp} 70$ are colored in green for eHsp70 ATP ${ }_{\text {NBD }}$ and in pink for eHsp70 ${ }^{\text {ATP }}$ SBD. The $_{\text {S }}$ cleft that the interdomain link binds is indicated by dashed circles. 
a
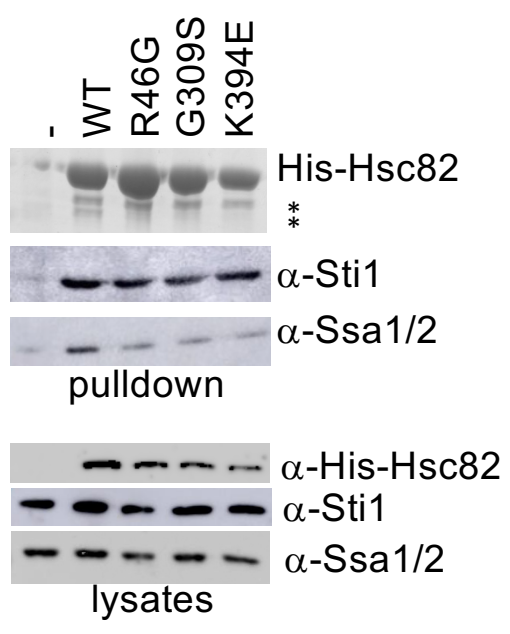

b

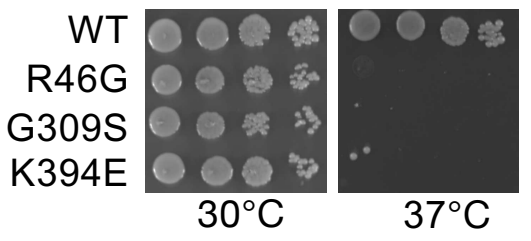

C

\begin{aligned} & \multicolumn{3}{c}{ Gal-Ura } \\ & WT v-src \\ & WT - \\ & R46G + \\ & G309S + \\ & K394E + \\ &+\end{aligned}

Extended Data Fig. 10 | Mutations that disrupt Hsp90:Hsp70 interactions in yeast. a, His-Hsc82 complexes were isolated from yeast and analyzed by SDS-PAGE and visualized by Coomassie staining and immunoblot analysis. Yeast proteins: Sti1=Hop; Ssa1/2=Hsp70; Hsc82=Hsp90ß. b, Plasmids expressing wild-type or mutant Hsc82 were expressed as the sole Hsp90 in JJ816 (hsc82hsp82) cells. Growth was examined by spotting 10-fold serial dilutions of yeast cultures on rich media, followed by incubation for two days at $30^{\circ} \mathrm{C}$ or $37^{\circ} \mathrm{C}$. c, Strains expressing WT or mutant HSC82 were transformed with a multicopy plasmid expressing GAL1-v-src (pBv-src) or the control plasmid (pB656) (Dey et al. 1996). Yeast cultures were grown overnight at $30^{\circ}$ in raffinose-uracil drop-out media until mid-log phase. $20 \%$ galactose was added to a final concentration of $2 \%$. After six hours, cultures were serially diluted 10 -fold onto uracil drop out plates containing galactose. Plates were growth for $2-3$ days at $30^{\circ} \mathrm{C}$. 


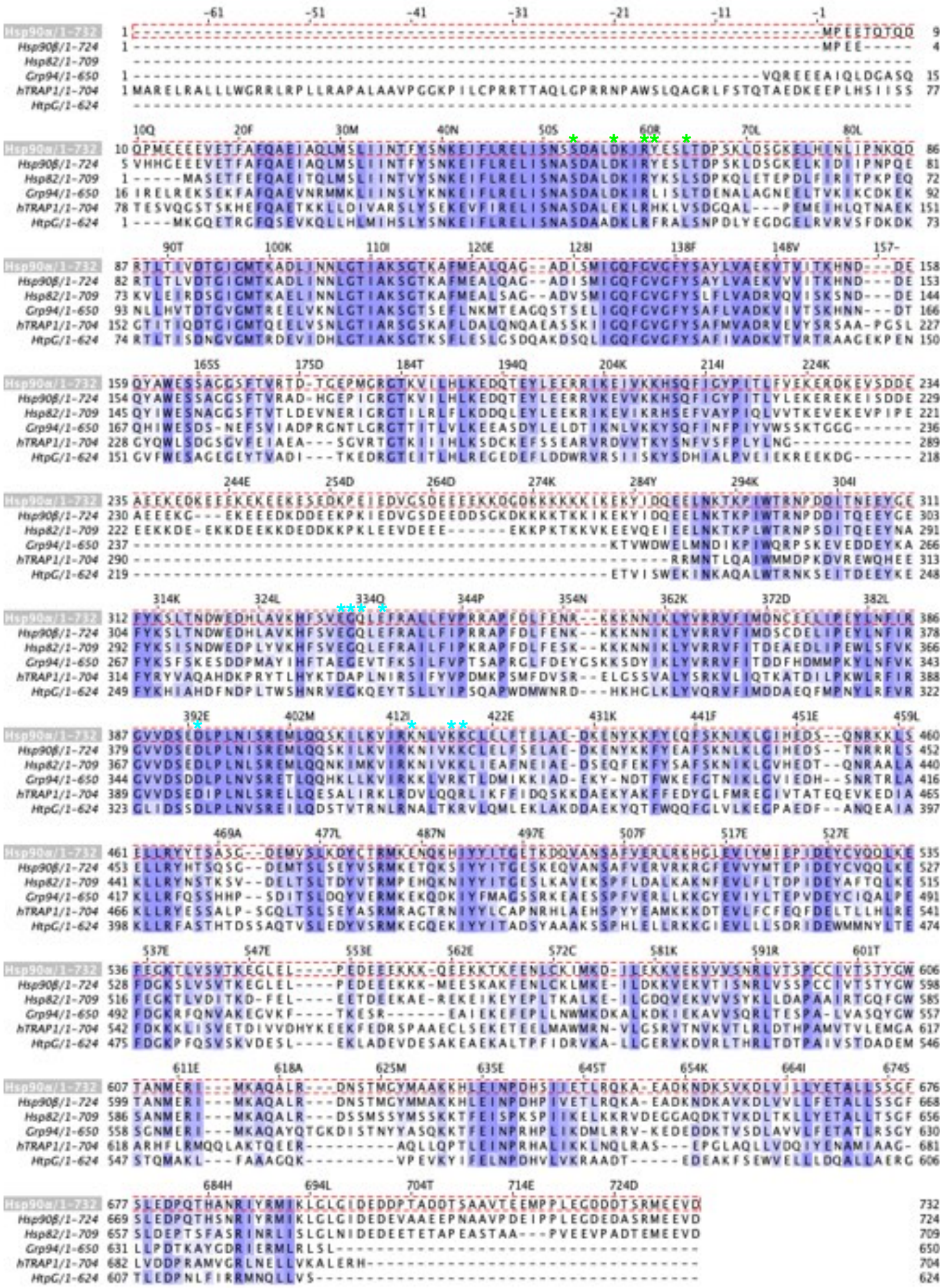

Extended Data Fig. 11 | Sequence alignments of Hsp90 paralogs and homologs. Conserved residues are highlighted in purple. Key residues involved in Hsp90:Hsp70 interface I and II are marked with cyan and green asterisks, respectively. Hsp90a, human stress-induced; Hsp90ß, human constitutively expressed; Hsp82, S. cerevisiae Hsp90; Grp94, human endoplasmic reticulum Hsp90; hTRAP1, mitochondrial Hsp90; HtpG, E. coli Hsp90. Multiple sequence alignments were generated using Clustal Omega and displayed using Jalview with BLOSUM62 Score color scheme. 


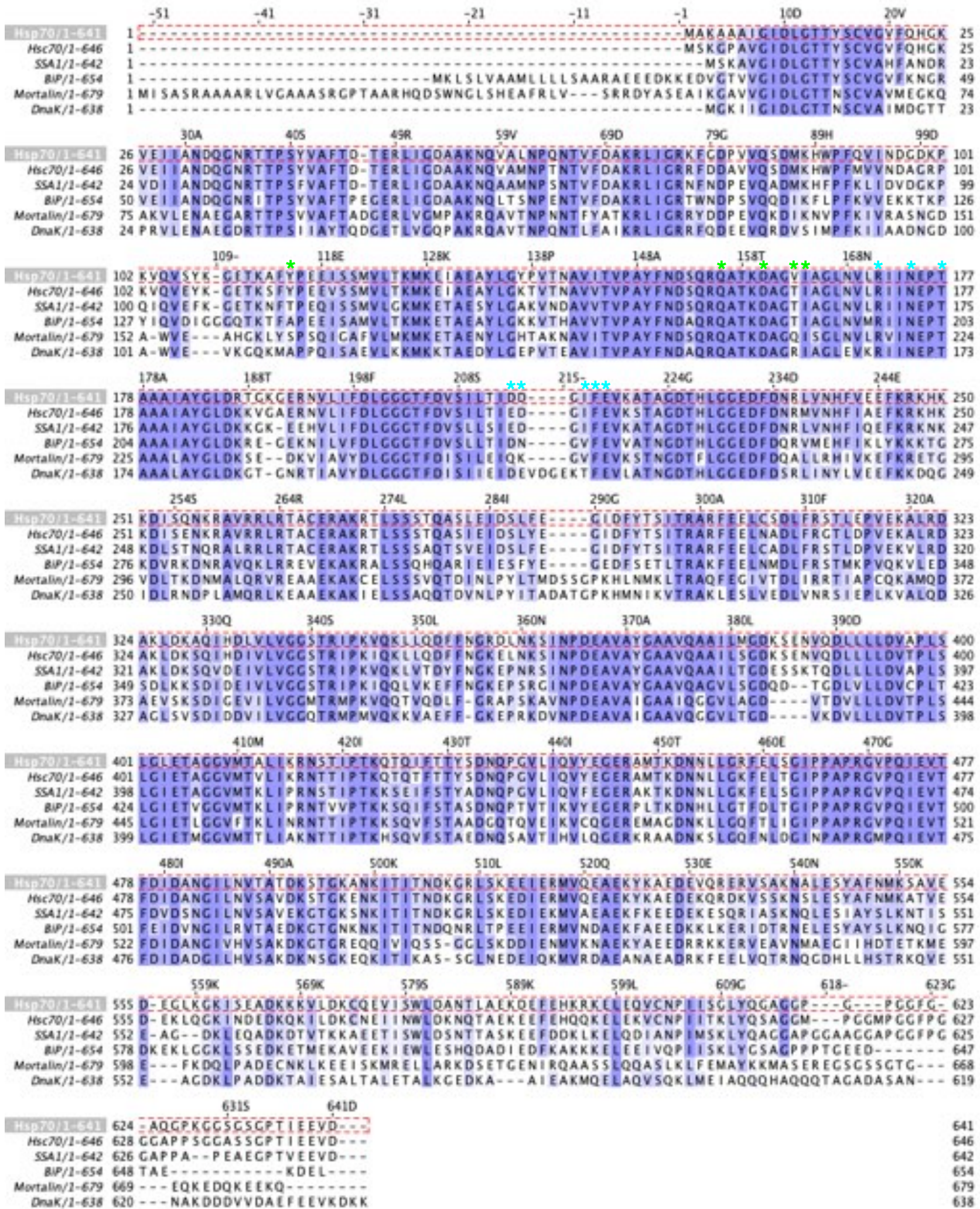

Extended Data Fig. 12 | Sequence alignments of Hsp70 paralogs and homologs. Conserved residues are highlighted in purple. Key residues involved in Hsp90:Hsp70 interface I and II are marked with blue and red asterisks, respectively. Hsp70, human stress induced; Hsc70, human constitutively expressed; SSA1, S. cerevisiae Hsp70; BiP, human endoplasmic reticulum Hsp70; Mortalin, mitochondrial Hsp70; DnaK, E. coli Hsp70. Multiple sequence alignments were generated using Clustal Omega and displayed using Jalview with BLOSUM62 Score color scheme . 

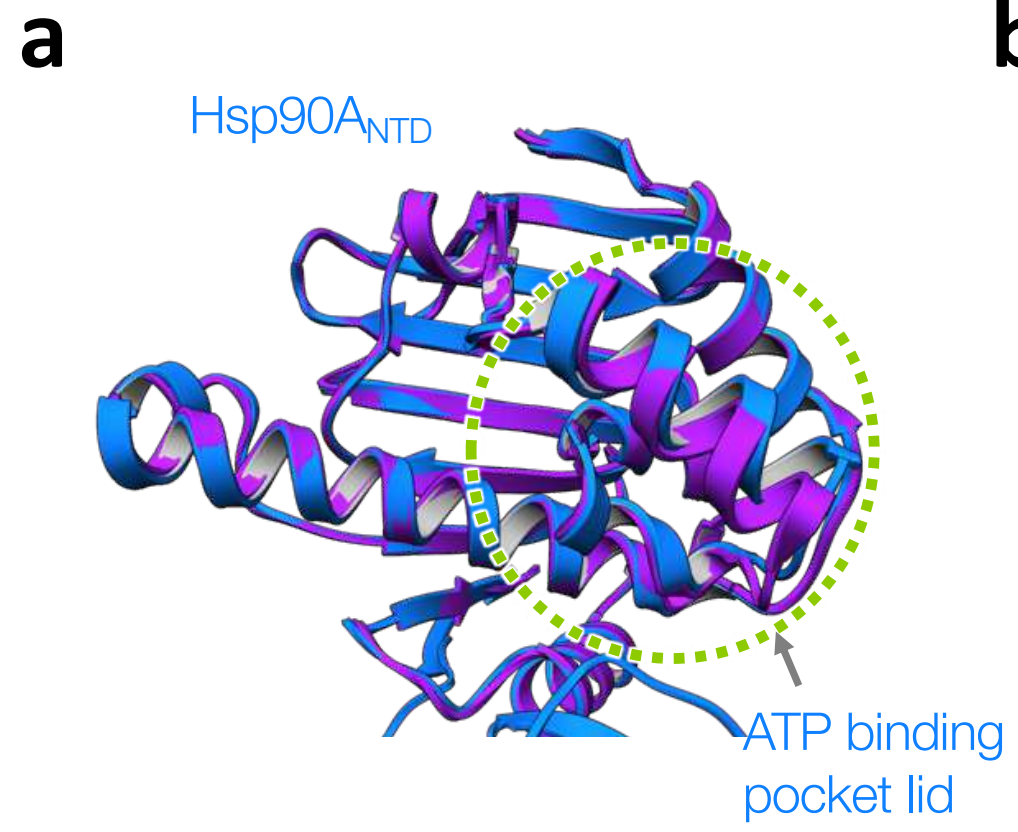

b
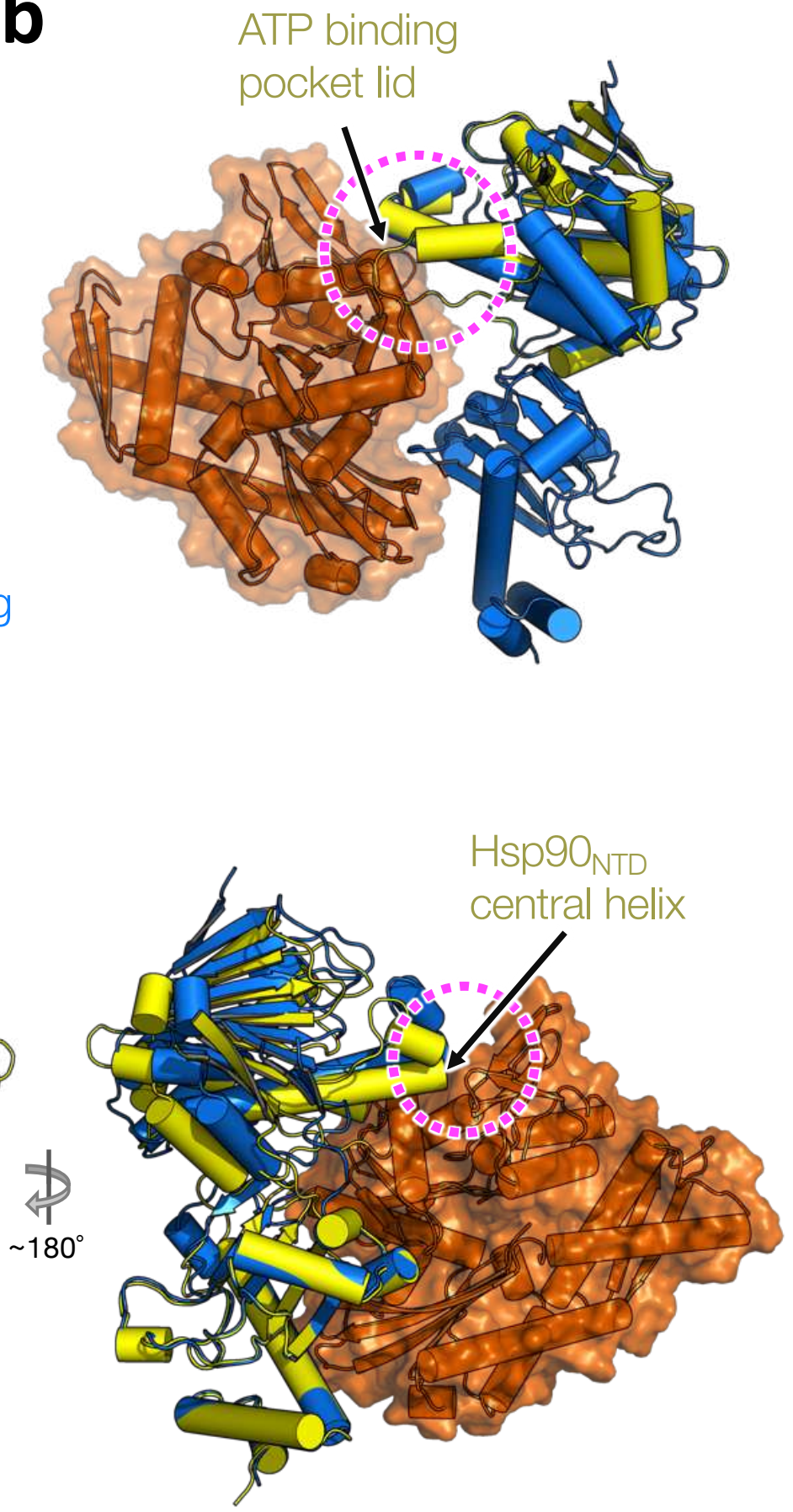

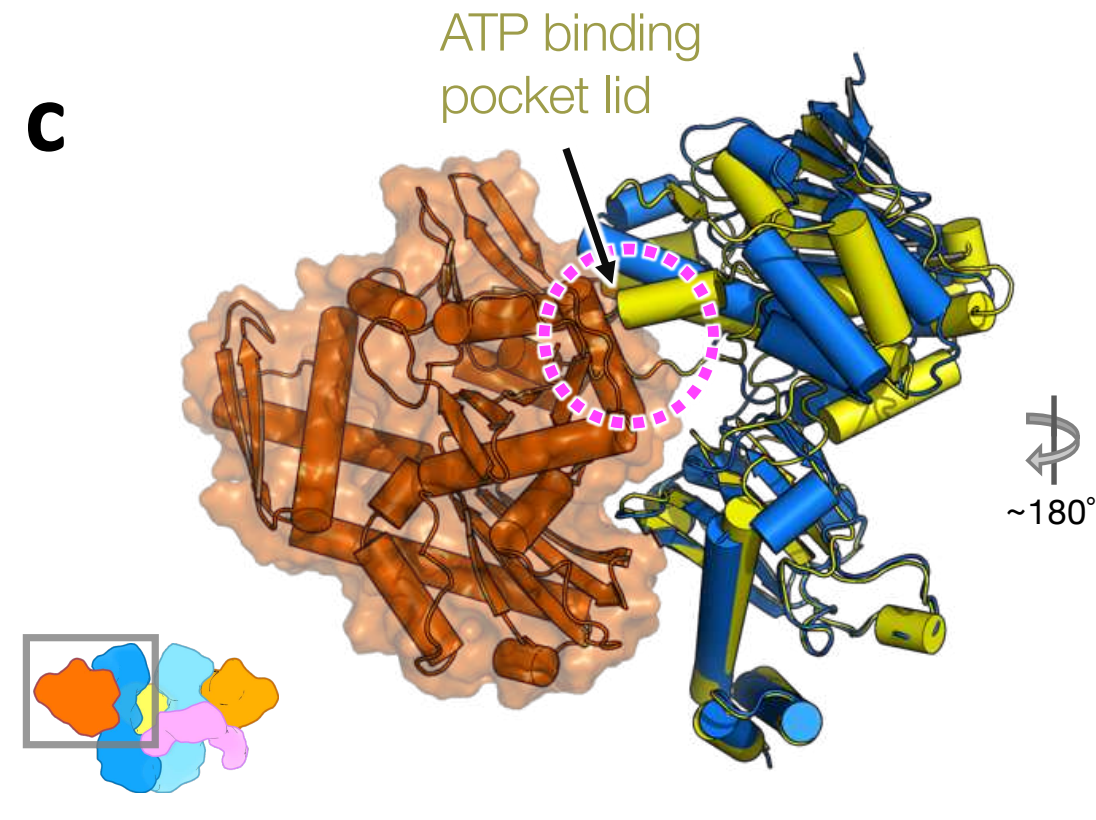

\section{ATP binding}

pocket lid

Extended Data Fig. 13 | The Hsp90ATP conformation is incompatible with the loading complex. a, Overlay of the crystal structure of Apo Hsp90 fragment (purple; PDB ID: 3t0h) to the Hsp90A (dark blue). Green circle highlights the open lid. b. Closure of the ATP pocket lid in the ATP state of $\mathrm{Hsp} \mathrm{N}_{\text {NTD }}$ (the Hsp90a structure from the GR-maturation complex is in yellow, ribbon representation) clashes (magenta circle) with the $\mathrm{Hsp} 70_{\mathrm{NBD}}$ (orange, surface and ribbon representation) in the loading complex. The NTD fragment of Hsp90ATP is aligned with the NTD of the Hsp90 in the loading complex. c, Superimposition of the ATP state of Hsp90 NTD-MD fragment (yellow) to the Hsp90A (dark blue) at the MD. Magenta circles indicate steric clashes of the ATP state of the Hsp90 NTD to the Hsp70 NBD (orange). 


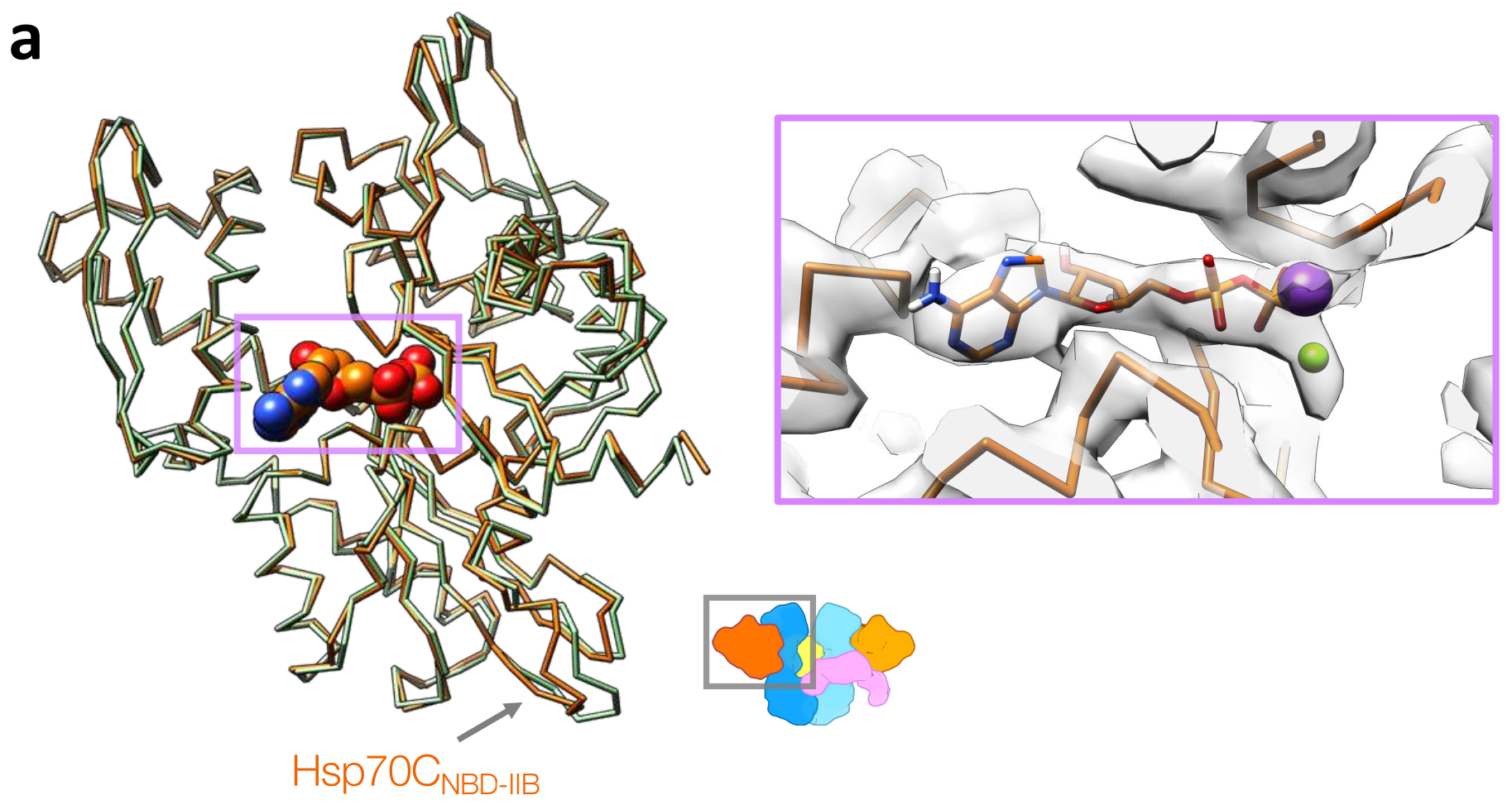

b

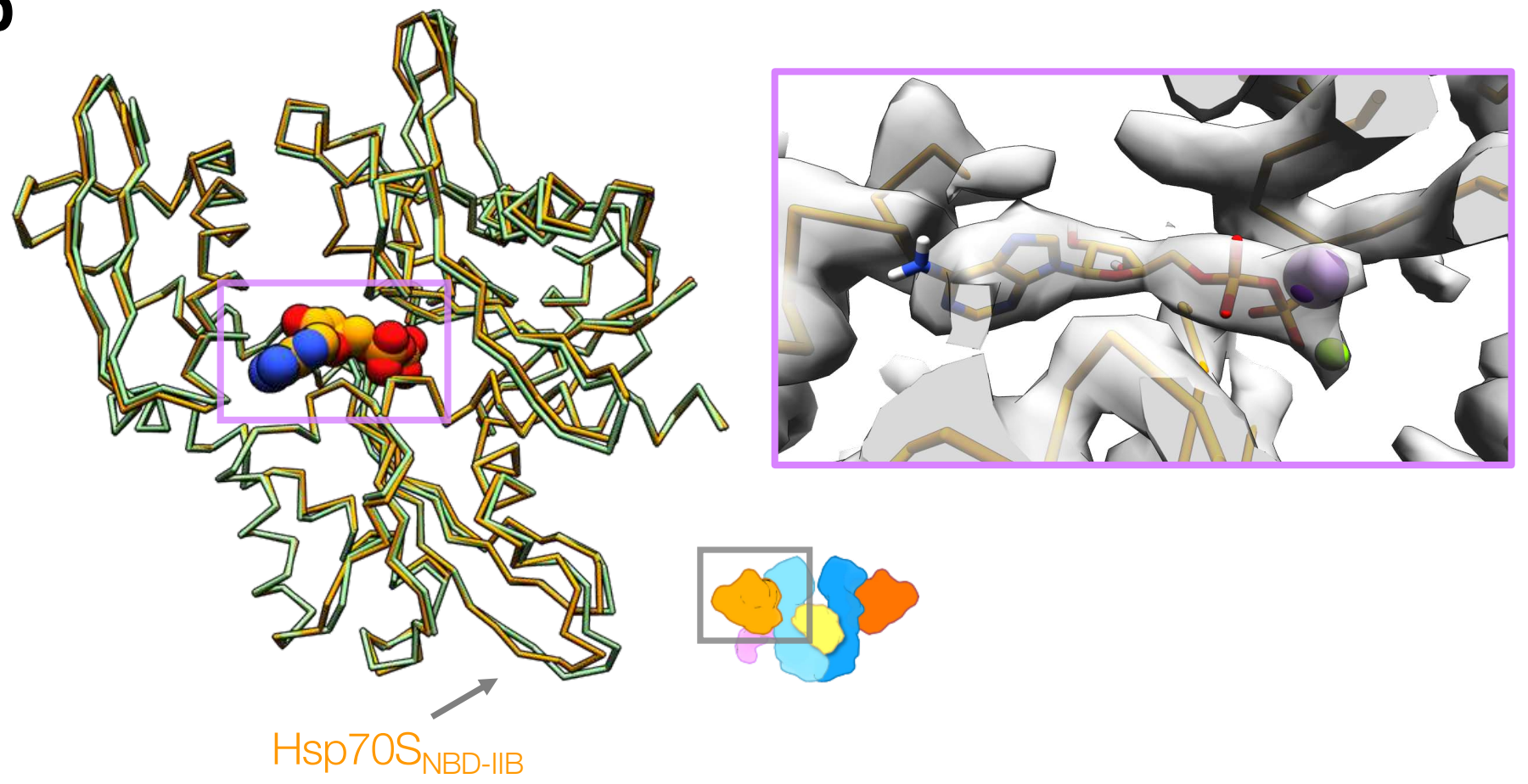

Extended Data Fig.14 | Marked deviation from the Hsp70 ${ }_{\mathrm{NBD}-I \mathrm{~B}}$ to the crystal structure. a,b, Superposition of human ADP-bound crystal structure of Hsp70 (green; PDB ID: 3ay9) to the Hsp70C (a, dark orange) and Hsp70S (b, light orange) with backbone chain-trace representation. The purple rectangles highlight the bound ADPs (sphere representation) and the corresponding densities (right). Two metal ions were found in the Hsp $70_{\mathrm{NBD}}$. Based on the buffer compositions and the 3AT9 crystal structure, the two metal ion densities were assigned to be Potassium (purple sphere) and Magnesium (green sphere). 
a

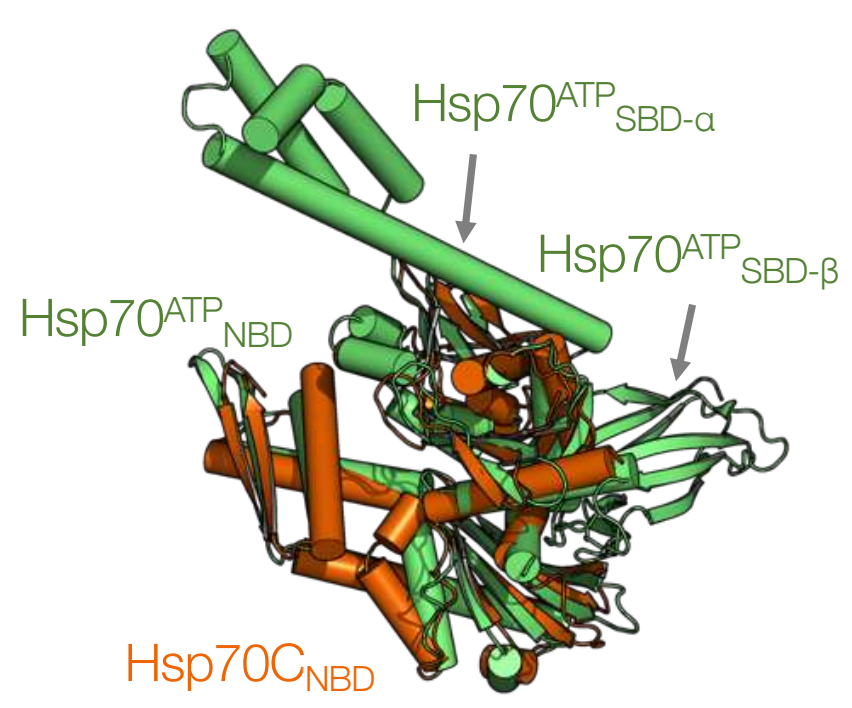

$$
180^{\circ}
$$

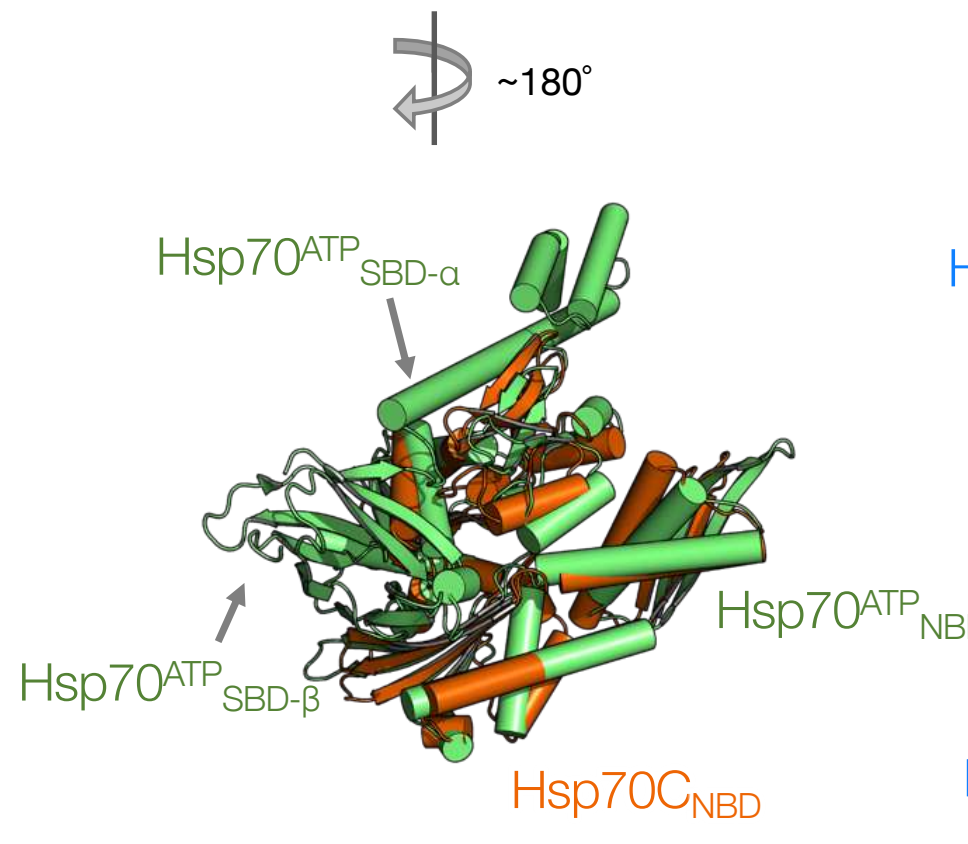

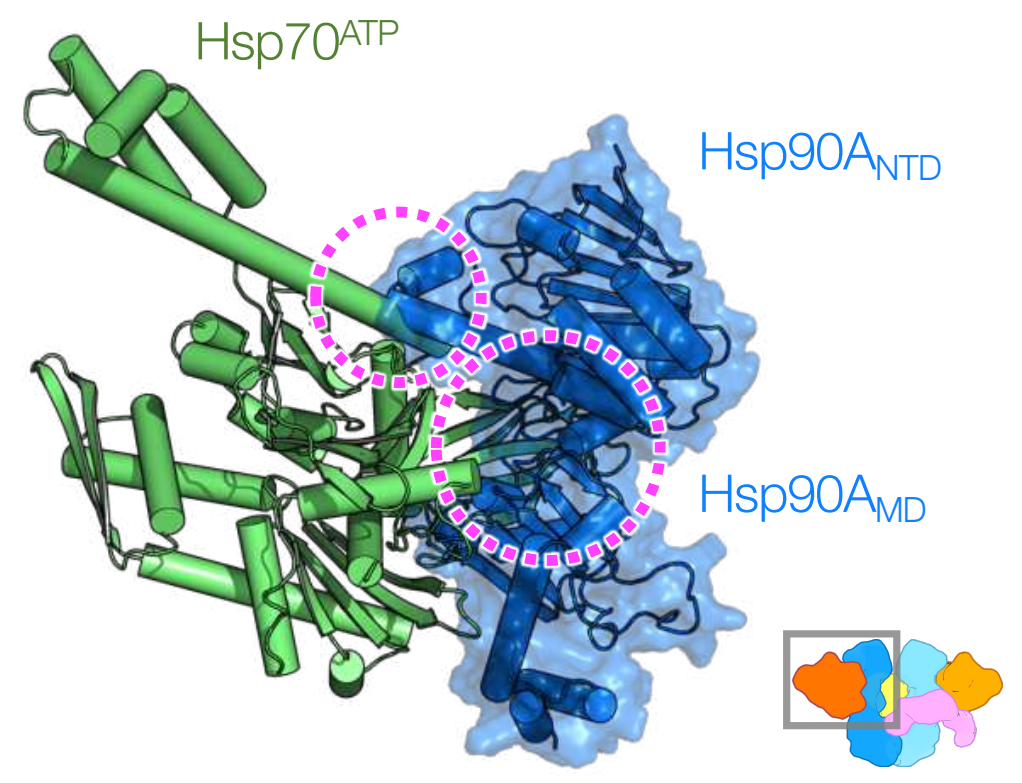

$\mathrm{Hsp}_{\mathrm{s}} \mathrm{A}_{\mathrm{MD}}$
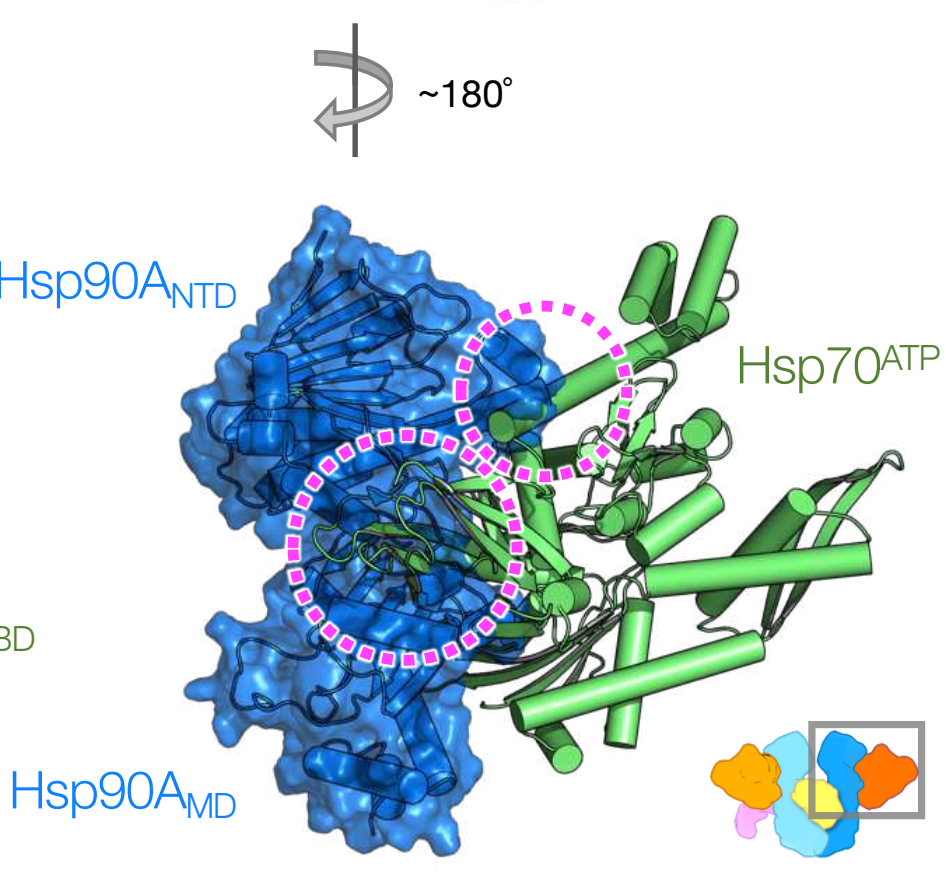

Extended Data Fig. 15 | The Hsp70 ATP conformation is incompatible with the loading complex. a, Superposition of the Hsp70 ATP conformation (green; PDB ID: 4B9Q) to the Hsp70C ${ }_{\text {NBD }}$ (dark orange). Arrows indicate the two subdomains of Hsp70ATP which cause serious steric clashes with the Hsp90 in the loading complex shown in (b). b. The superimposed Hsp70ATP shown in (a) is fixed and the Hsp90 (dark blue; surface/ribbon representation) of the loading complex is present. Magenta circles highlight steric clashes caused by the two subdomains of Hsp70ATP ${ }_{\text {SBD }}$ (green) to the Hsp90A NTD-MD. 
a

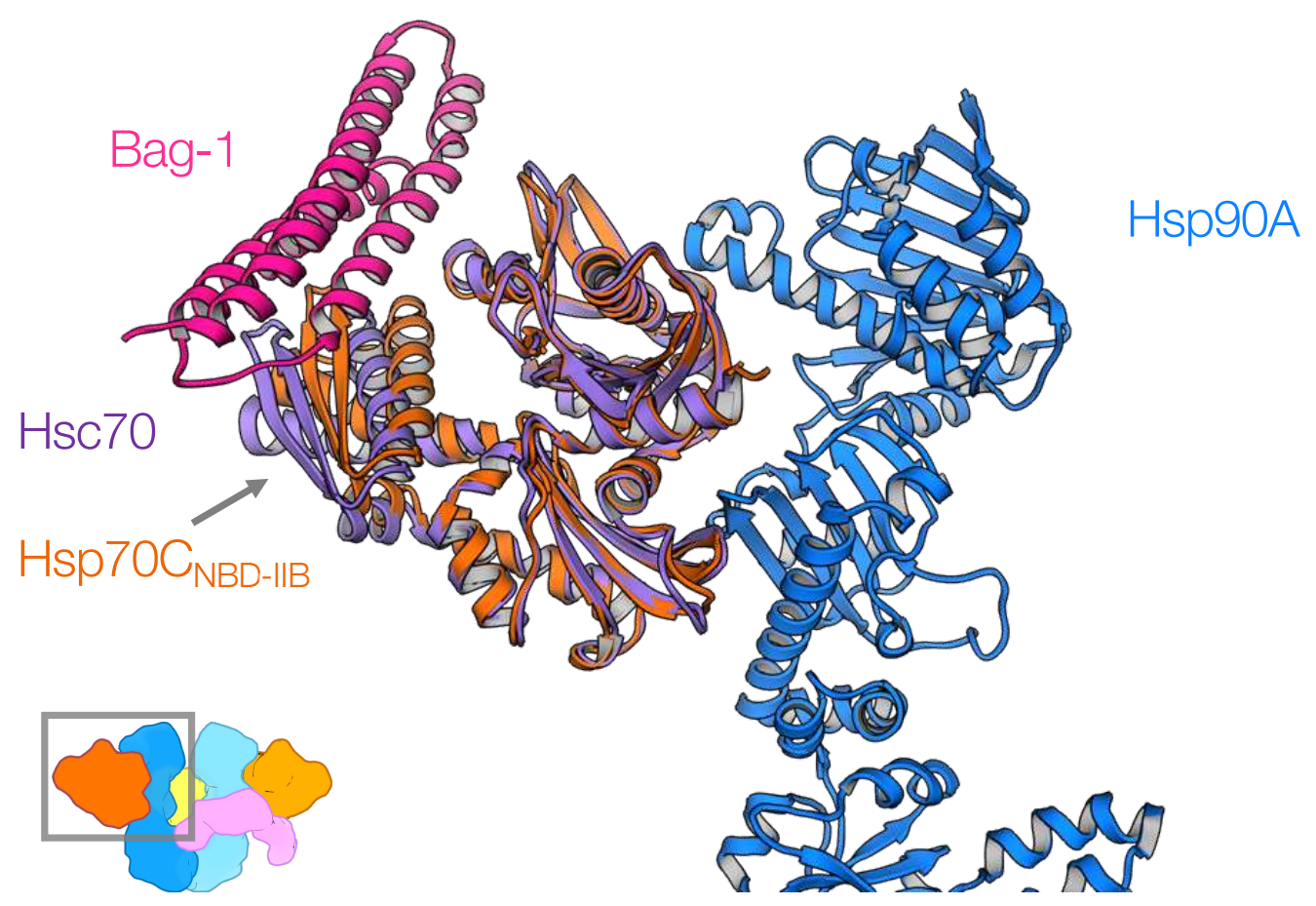

b

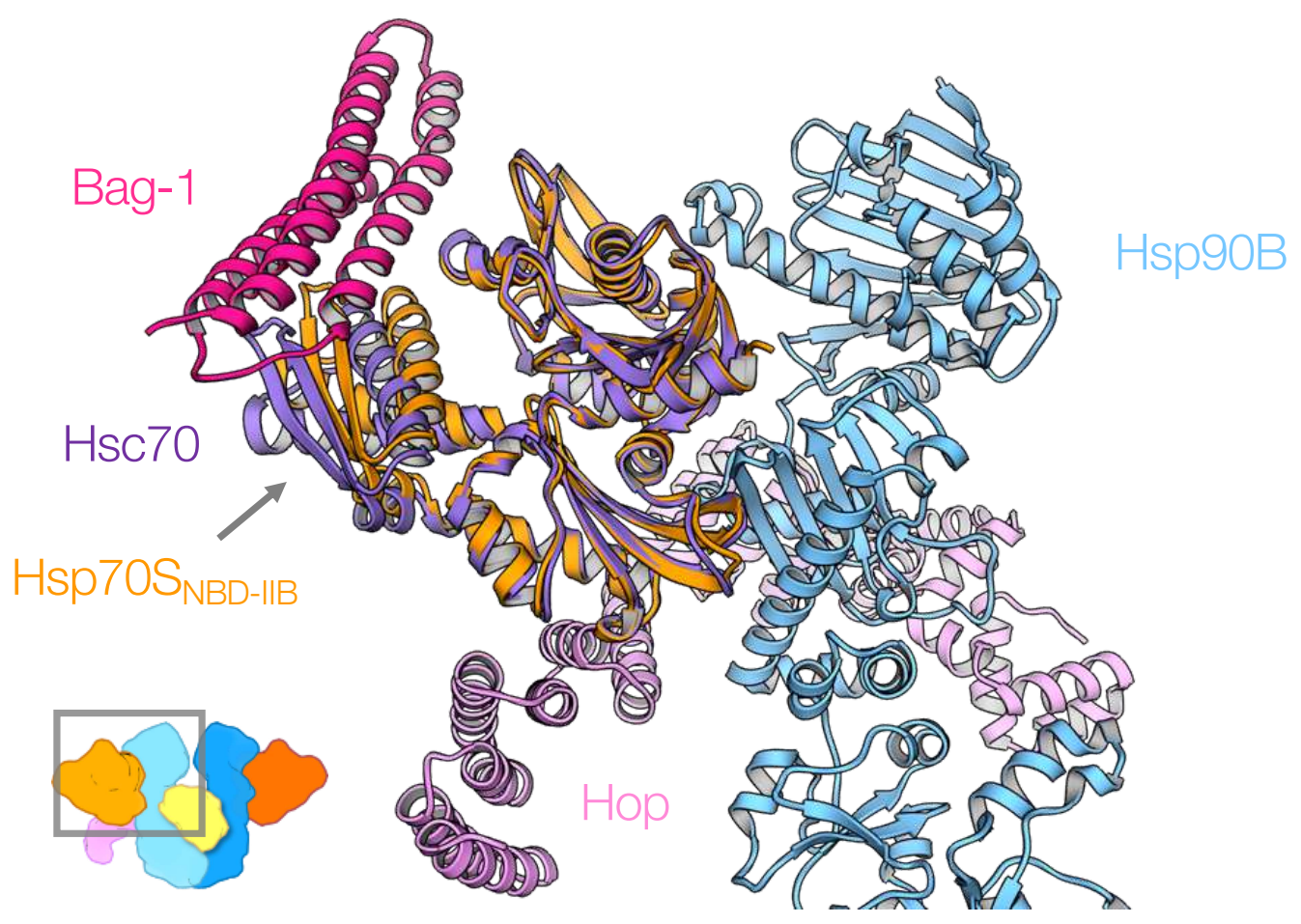

Extended Data Fig. 16 | The canonical NEF binding sites are available in both of the Hsp70s. a,b, Superposition of the crystal structure (PDB ID: 1HX1) of the Bag-1:Hsc70 complex with the Hsp70C (a) and Hsp70S (b) on the loading complex. The Bag-1 and Hsc70 from the crystal structure are colored with magenta and purple, respectively. Components of the GR-loading complex are colored as in other figures and as labelled. 
a

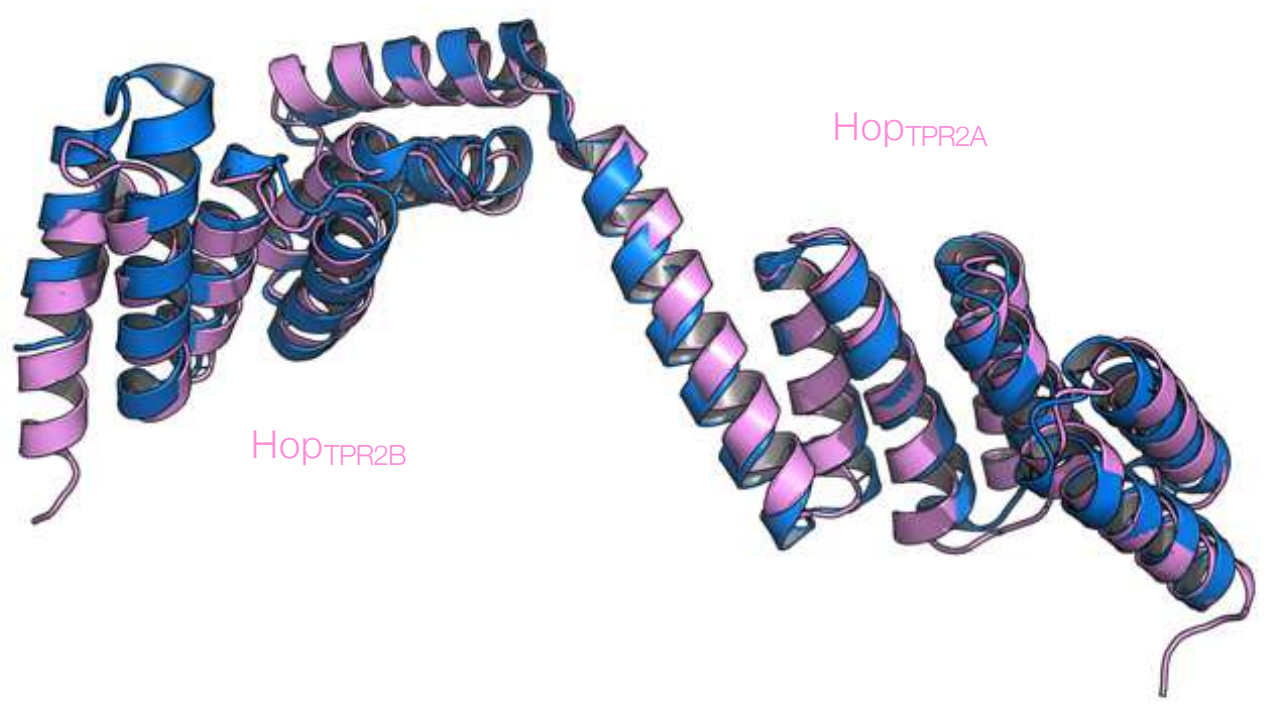

b

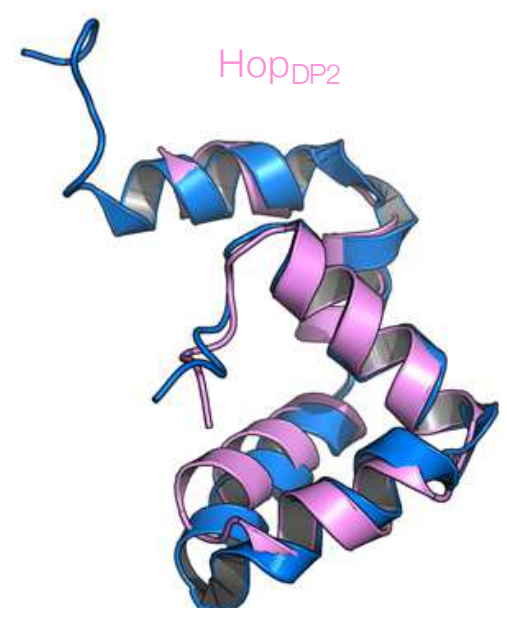

C

d
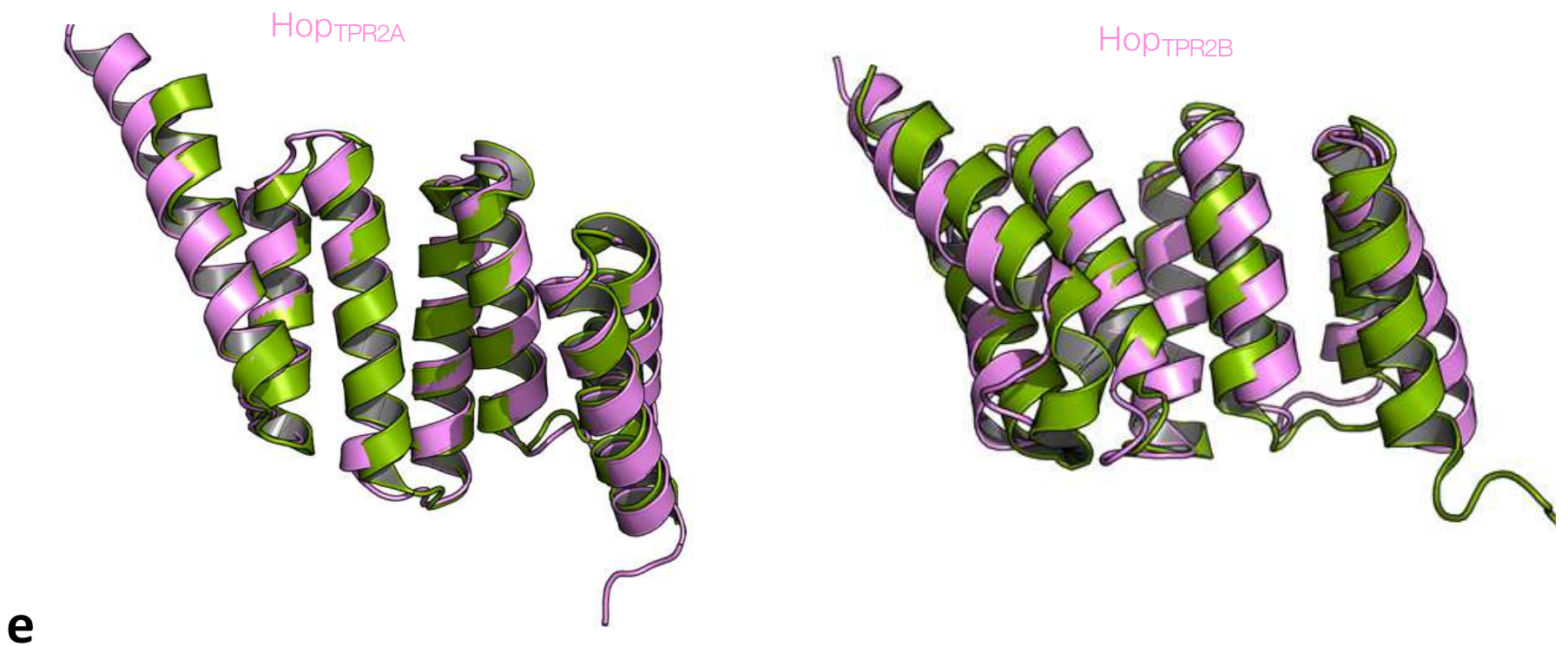

e

10

20

30

40

50

60

hHopDP2,/1-65 HDSPEDV K yHopDP2,/1-65 NETPEETYQRAMKDPEVAA I MQDP VMQS I LQQAQQNPAA LQEHMKNPEVFKK I QTL I AAG I I RTG

Extended Data Fig. 17 | Comparison of the Hop TPR2A-TPR2B-DP2 $_{\text {structure with published experimental }}$

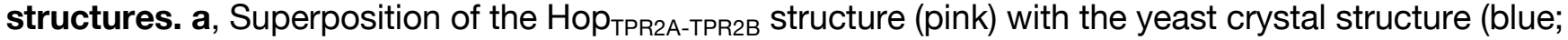
PDB ID: 3uq3). b, Superposition of the Hop $\mathrm{DP}_{2}$ (pink) with the yeast Hop $\mathrm{DP}_{2}$ NMR structure (blue; PDB ID: 2llw). c, Superposition of the Hop ${ }_{T P R 2 A}$ structure (pink) with the NMR structure (green; PDB ID: 2nc9). d,

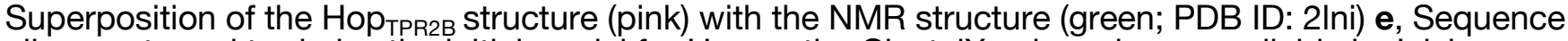
alignment used to derive the initial model for $\mathrm{Hop}_{\mathrm{DP} 2}$; the ClustalX color scheme available in Jalview was used to color the alignment. 
a

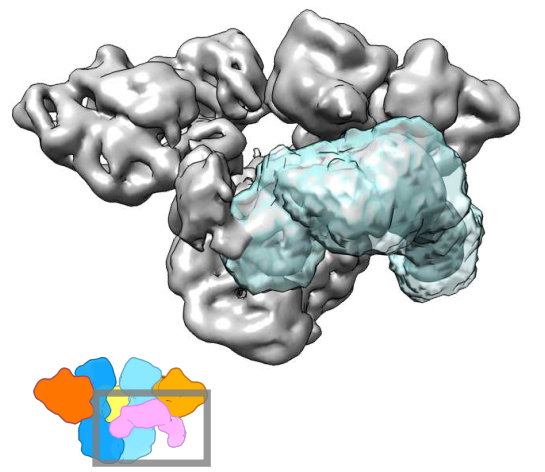

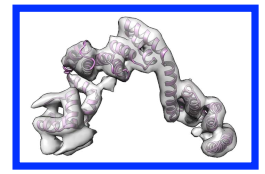
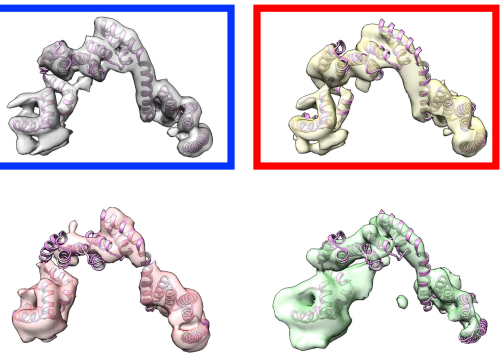
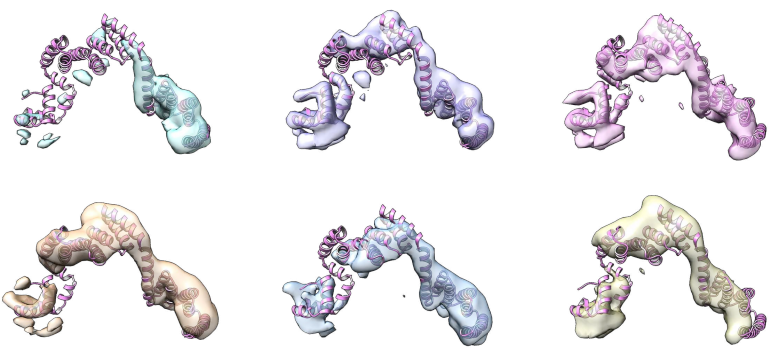

C
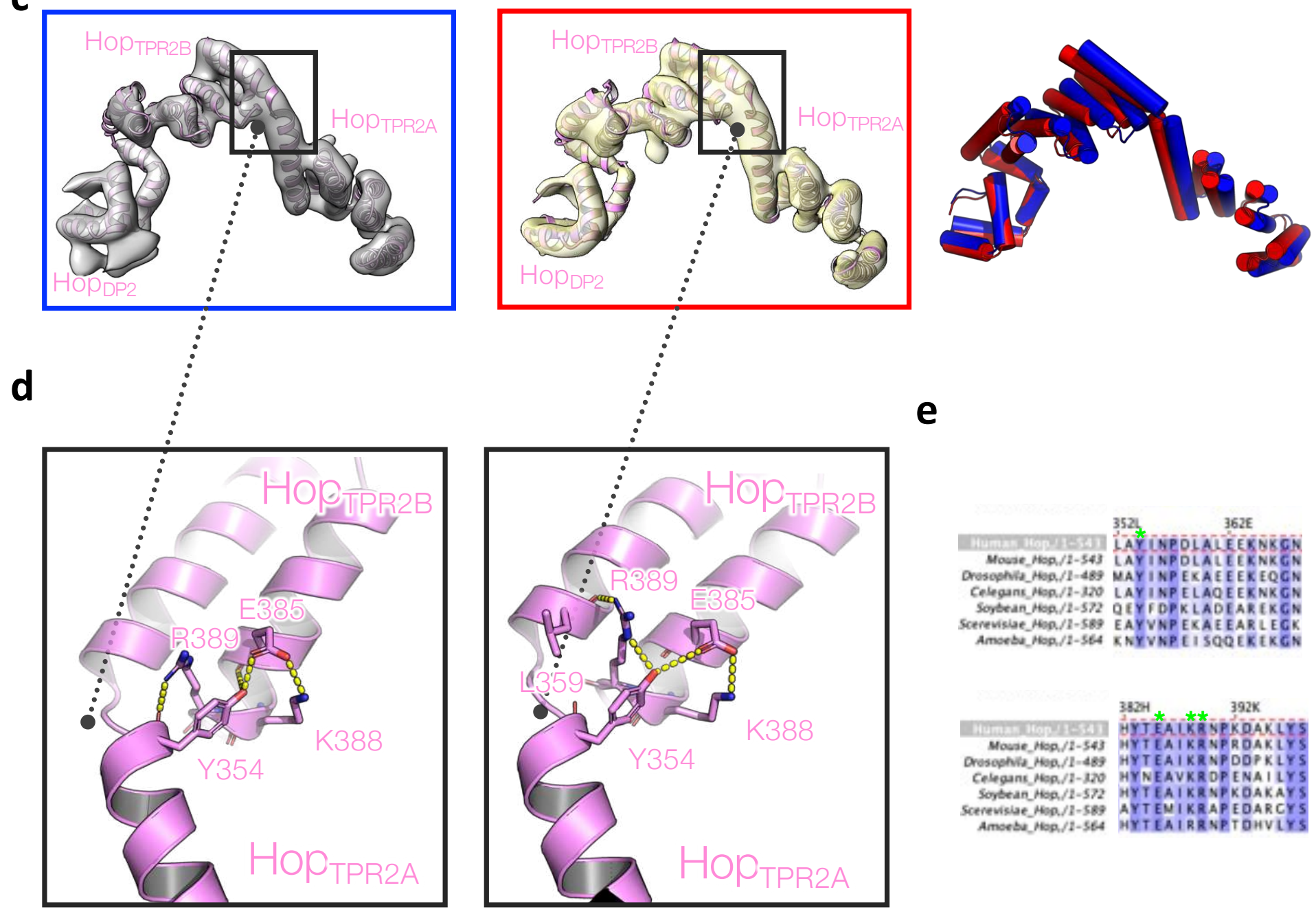

$\mathbf{e}$
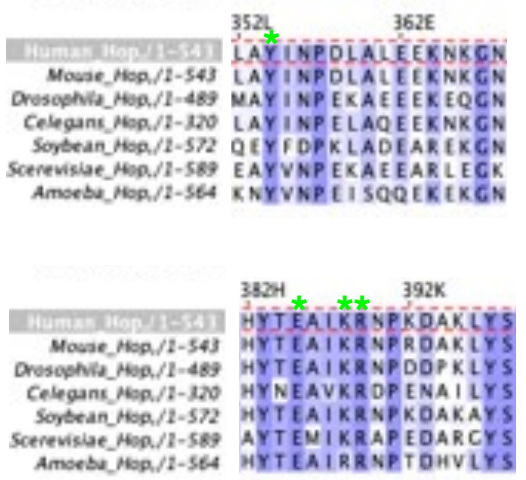

Extended Data Fig. 18 | Conformation dynamics of Hop TPR2A-TPR2B-DP2 revealed by focused classification (without alignment). a, The mask used for the focused classification. b, 10 classes from RELION focused classification. Ribbon model (pink) fitted into the highest resolution class (blue rectangle) is kept static as a reference frame for the rest of the 9 maps. The top 2 high-resolution classes are highlighted with boxes. c, Models refined into the top 2 high-resolution classes (left and middle), respectively. Overlaid models without superposition (right) from the left and middle panels. d, A network

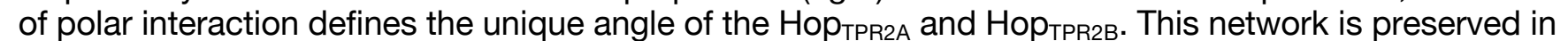
the two conformations. A similar network can also be found from the yeast homolog structure (PDB ID: 3uq3). e, Multiple sequence alignments of Hop from model systems. The residues involved in the network from (d) are indicated with green asterisks. Color scheme is BLOSUM62. 
a
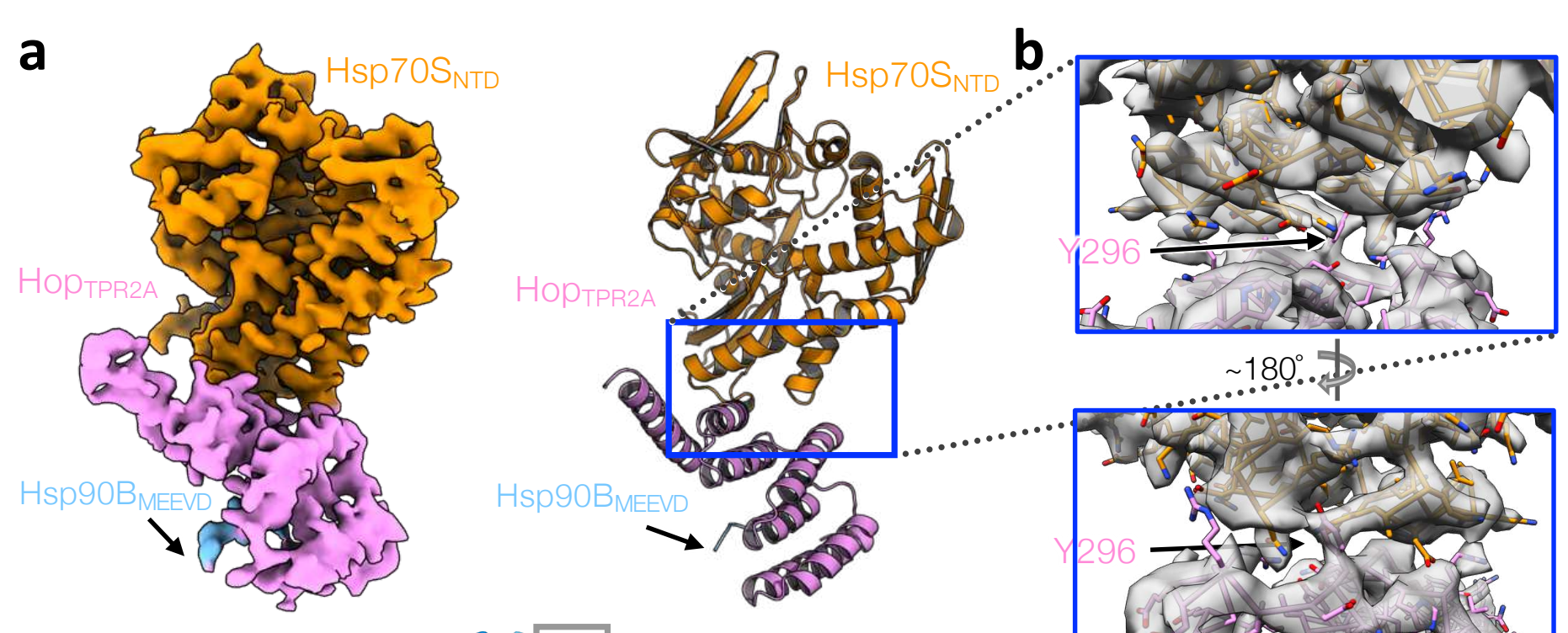

$$
\sim 90^{\circ} \text { 卉 }
$$
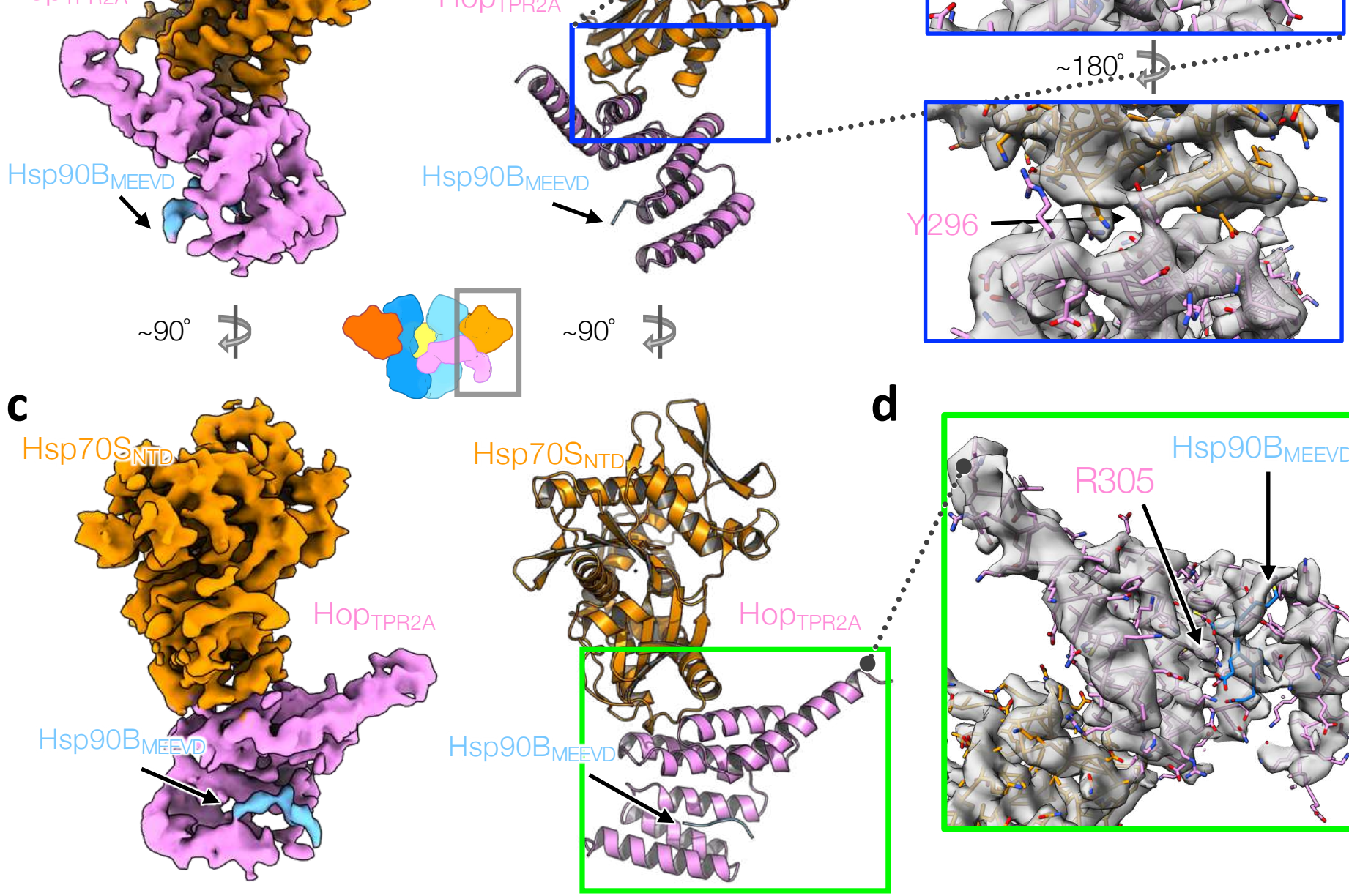

d

$\mathbf{e}$

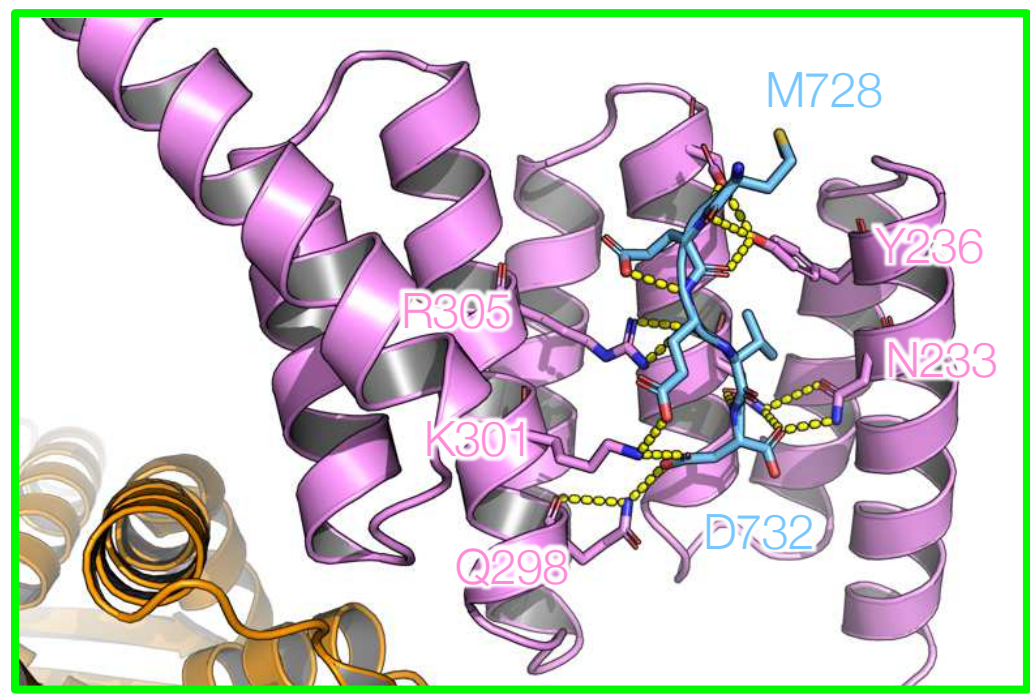

f

$29 \mathrm{FY}$

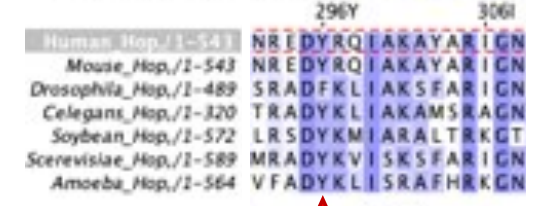

$\Delta$

Y296

Extended Data Fig. 19| The atomic interactions of Hsp70S:Hop TRP2A:Hsp90B ${ }_{\text {MEEVD }}$ in the GR-loading complex. a,c, Left, the cryo-EM map from focused classification and refinement. Right, the atomic model with the corresponding views from the left. b. Close-up views of the Hsp70S ${ }_{\text {NTD }}: H_{\text {TPR2A }}$ interface with the atomic model fit into the density. Sidechain density for Y296 from Hop TPR2A is indicated with the arrow. d, Close-up view of the HopTPR2A. e, Close-up view of the atomic interactions of the MEEVD fragment from Hsp90B (light blue) and HoptPR2A (pink). Polar interactions are depicted with dashed lines. f, Sequence alignments of Hop indicate Y296 (red triangle) is highly conserved. Color scheme is BLOSUM62. 
a

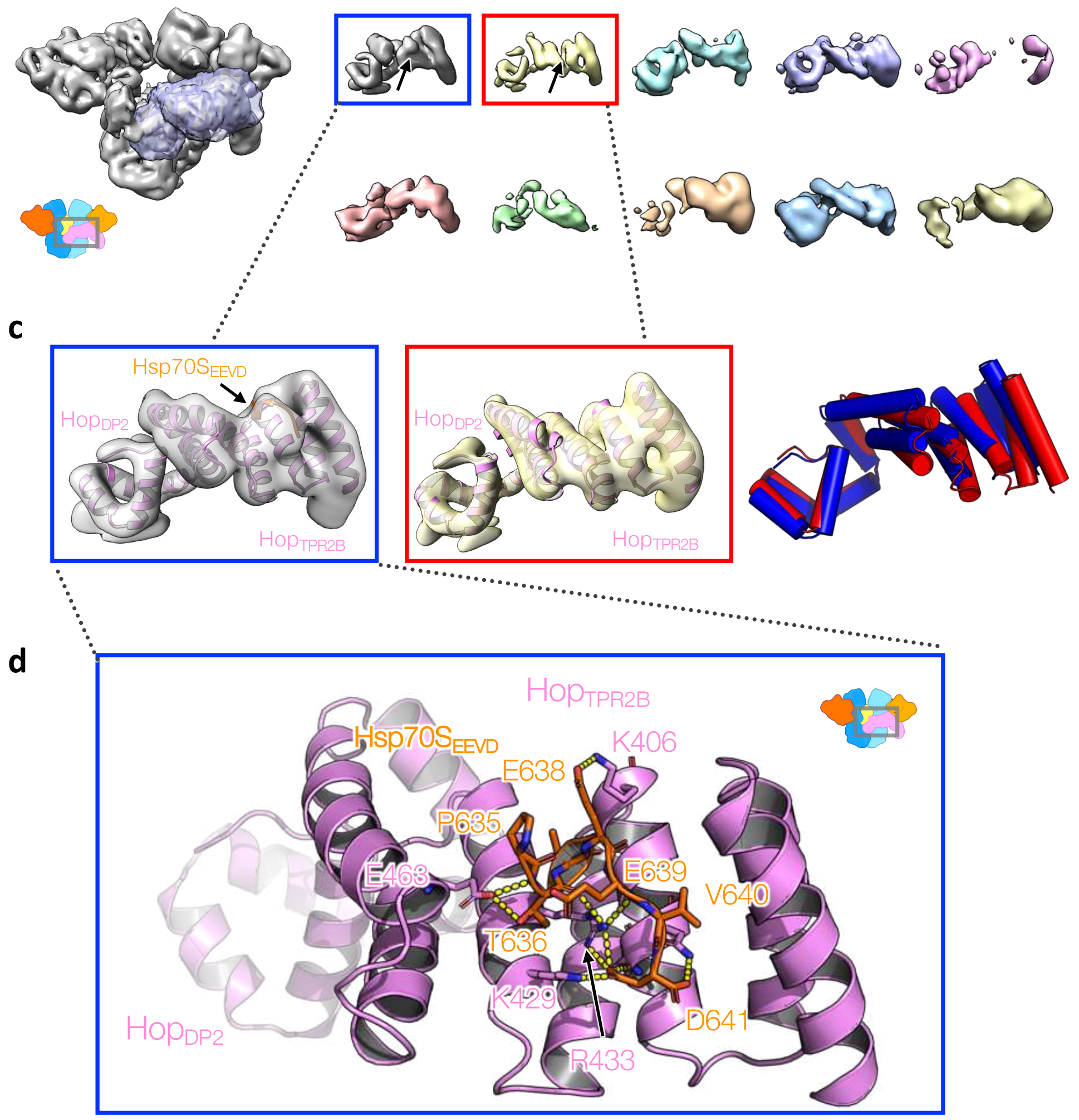

Extended Data Fig. 20 | Hsp70S EEVD-bound Hop $_{\text {TPR2B-DP2 }}$ revealed by focused classification (without alignment). a, The mask used for the focused classification. $\mathbf{b}$, The 10 classes from the focused classification. The top 2 high-resolution classes are highlighted with boxes. c, Models refined into the top 2 high-resolution classes (left and middle), respectively. Left, the highest resolution class has extra EEVD density (arrow) from Hsp70S, whereas the second-highest resolution class has no density in the EEVD binding pocket (middle panel). Right, overlaid models without superposition from the left and middle panels. d, Refined atomic model of Hop TPR2B with Hsp70 was derived from the yeast homolog (PDB ID: 3UPV). Polar interactions are depicted with dashed lines. 

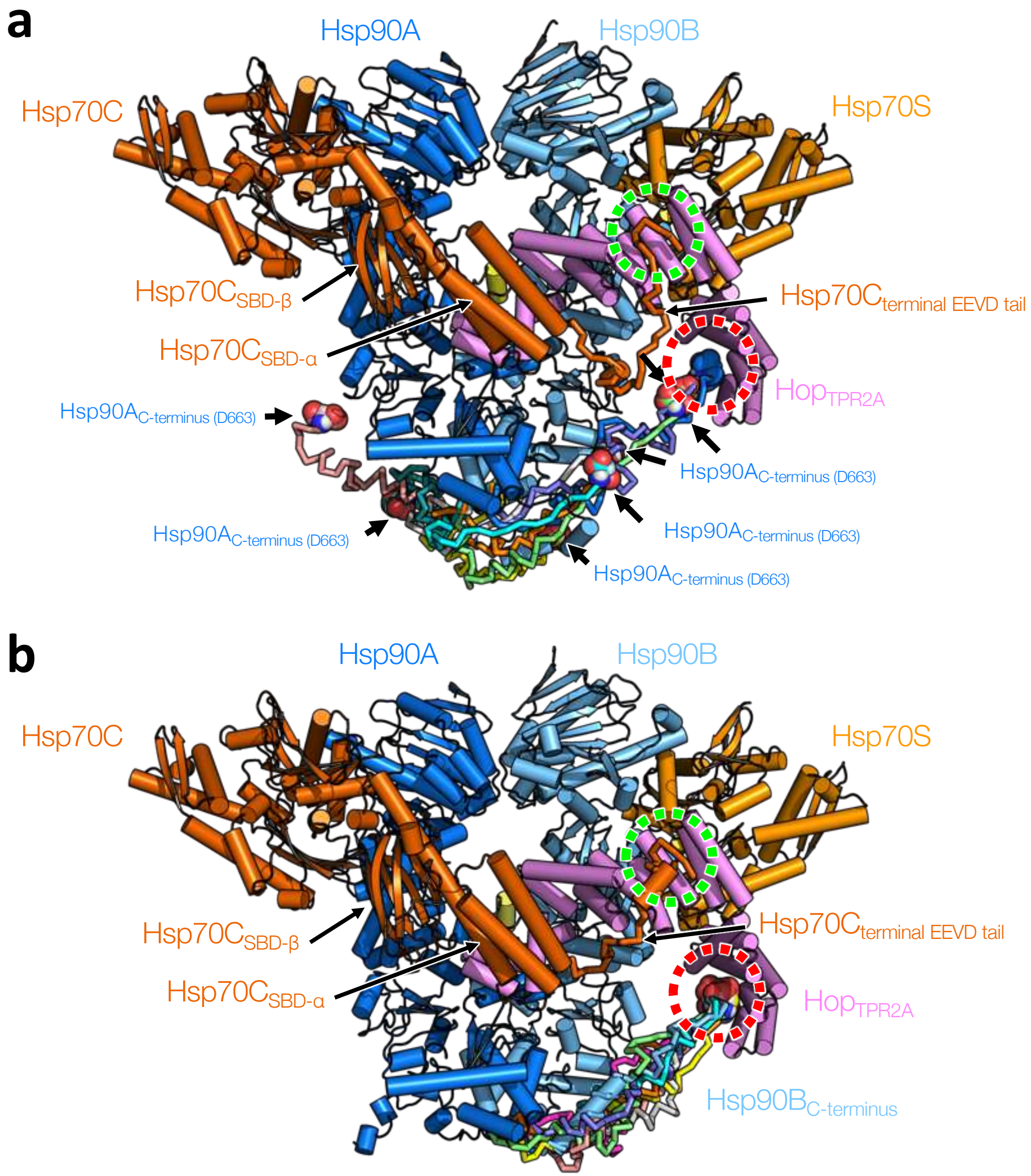

Extended Data Fig. 21 | Modeling the EEVD tails from Hsp90/Hsp70 to Hop TPR2A and Hop ${ }_{\text {TPR2B }}$, respectively. a,b, EEVD tail modeling starting with the loading complex model (shown in cartoon representation with the same color scheme) with a Hsp70/Hsp90 EEVD fragment bound to Hop TPR2A (red dashed circle) and Hop TPR2B $_{\text {(green }}$ dashed circle). Hsp70 ${ }_{\mathrm{SBD}-\mathrm{a}}$ and its following 30 tail was modeled to connect to the Hop $\mathrm{TPR}_{\mathrm{TB}}$ EEVD-binding pocket. Two separate loop closure jobs were assigned to Hsp90A (top) and Hsp90B (bottom), respectively. The top 10 lowenergy models are shown in ribbon representation with various colors and the very last residues of the MEEVD tail (D663) are shown in sphere representation with the black arrows pointed. Among the top 10 low-energy models, only 3 models from the Hsp90A job (top) successfully maintained the connection to HopTPR2A (red circle), whereas all 10 models from the Hsp90B job (bottom) were able to maintain the binding to Hop ${ }^{\text {TPR2A }}$ (red circle). 
a

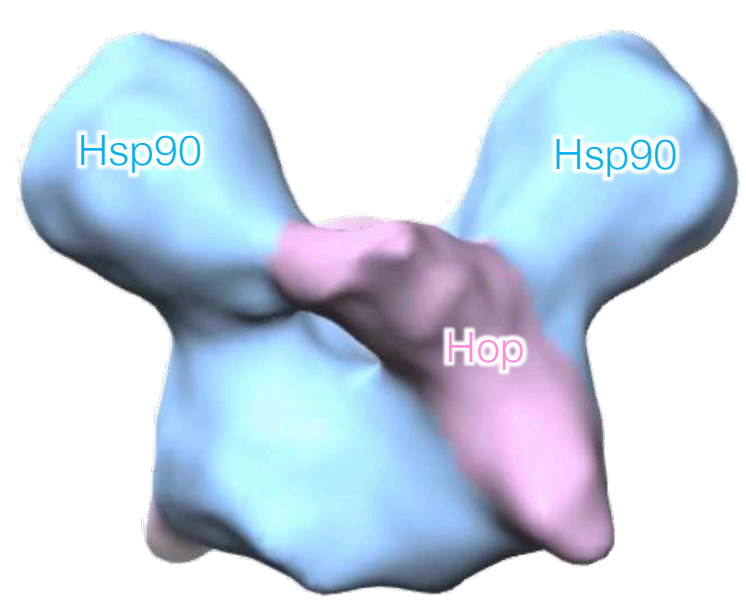

Hsp90:Hop cryo-EM map (z-flipped) b
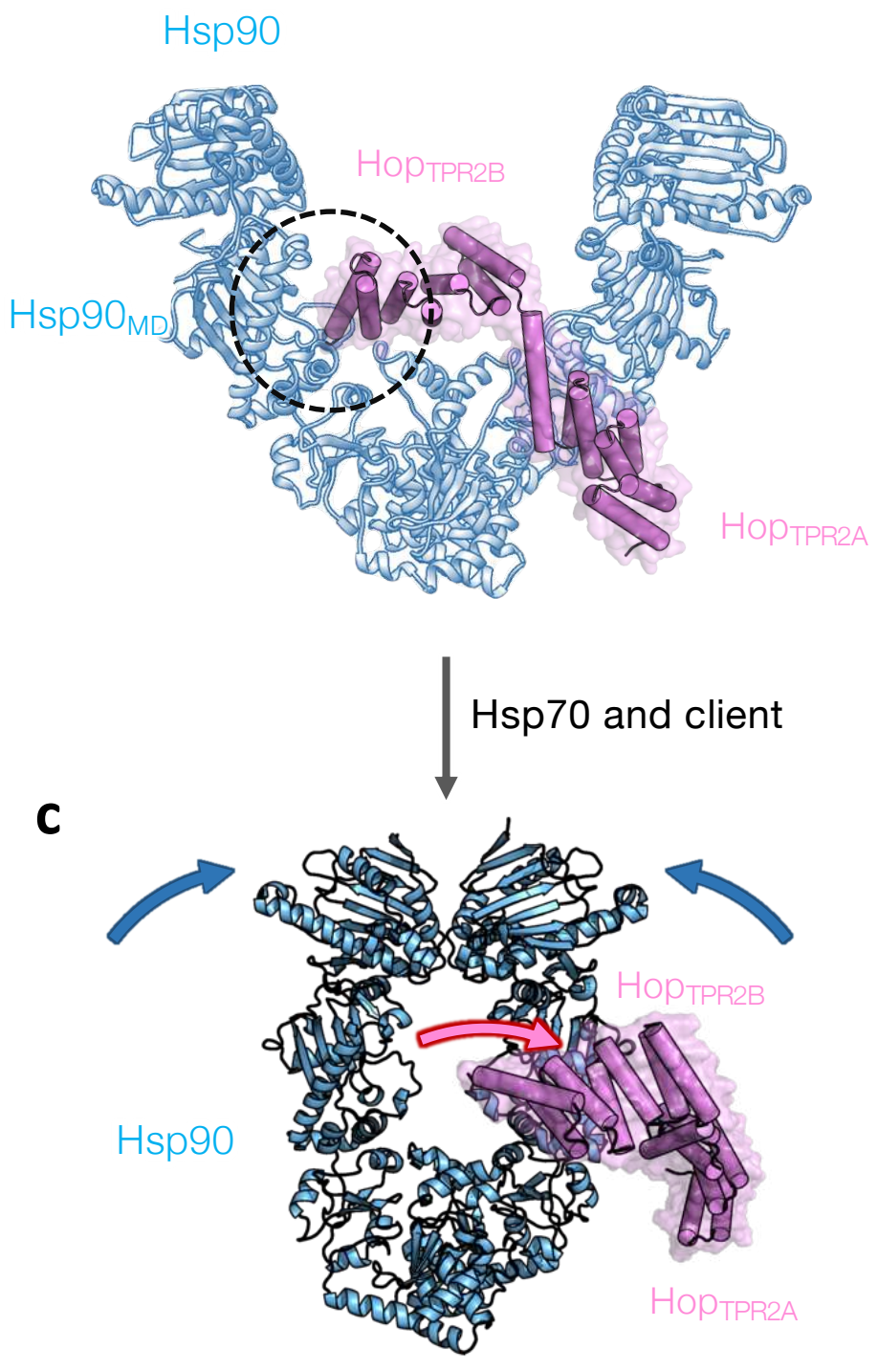

The Hsp90 and Hop

in the GR-loading complex

Extended Data Fig. 22 | Hop may first prepare Hsp90 for Hsp70 and client interaction. a, A mirror image of the 15-Å-resolution cryo-EM map of the Hsp90:Hop complex (Southworth \& Agard, 2014). In cryo-EM single-particle analysis, there is a $50 \%$ chance to get a mirror reconstruction and the correct handedness is difficult to distinguish in medium to low resolution. b, Guided by the flipped map and the GR-loading complex structure, now the Hop density (pink shade in (a)) can be interpreted as the TPR2A-TPR2B module of Hop as it possesses a unique dumb-bell-like structure. Dashed circle indicates a contact of $\mathrm{Hop}_{T P R 2 B}$ to $\mathrm{Hsp90} \mathrm{MD}_{\mathrm{MD}}$ in this state. c, The Hsp90 and Hop structure in the GR-loading complex. After interacting with Hsp70 and GR, the Hsp90 dimer and Hop TPR2A-TPR2B undergo conformational changes from the Hsp90:Hop complex, which are indicated with the blue and pink arrows, respectively. 
a
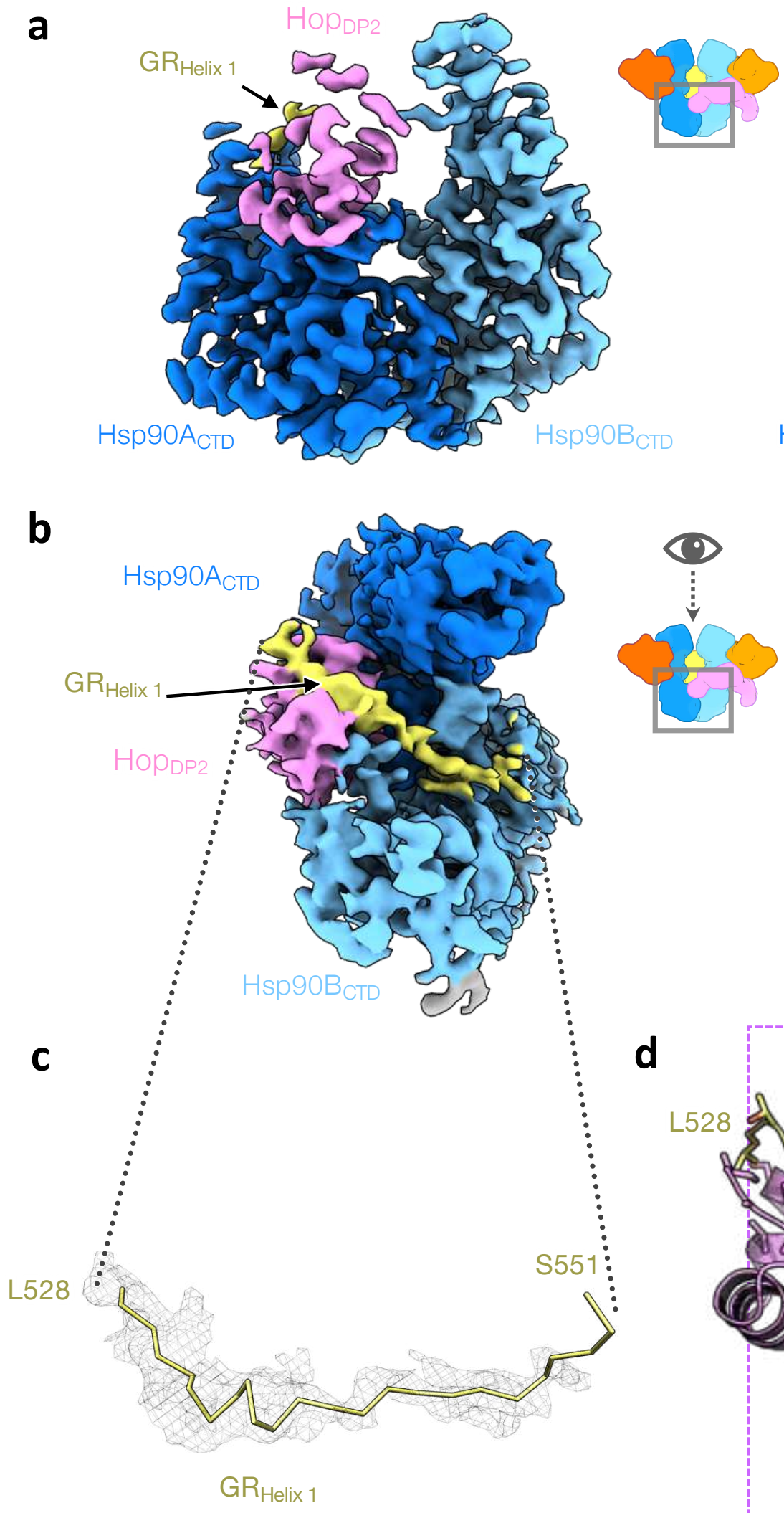
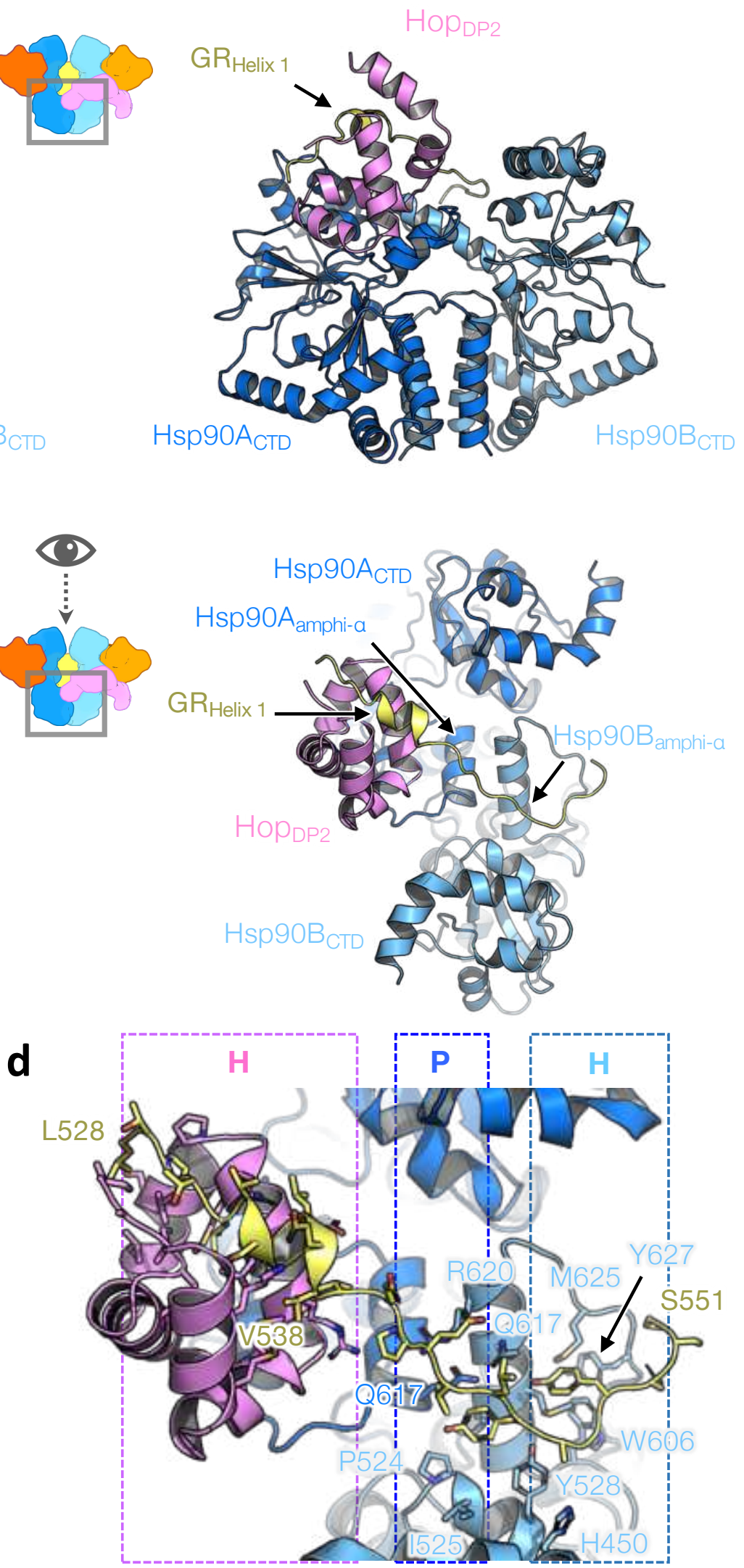

Extended Data Fig. 23 | GR Helix 1 motif's interactions with Hsp90 and Hop DP2. $^{\text {a, The }}$ focused map of the Hsp90AB ${ }_{C T D}: H_{0 p_{D P 2}}: G_{N \text {-term }}($ left) and the atomic model shown in representation (right). $\mathbf{b}$, The top view of the reconstruction and model shown in (a). c, The density (mesh) for the $\mathrm{GR}_{\mathrm{Helix} 1}$ motif (residues 528-551) gripped by Hsp90 and Hop $\mathrm{DP}_{2}$. d, The atomic interactions of the $\mathrm{GR}_{\mathrm{Helix} 1}$ motif with Hsp90 and Hop $\mathrm{DP}_{2}$. Residues in contact with the GR motif are shown in stick representation. The types of molecular interaction $\mathrm{Hsp} 90$ and Hop $\mathrm{DP}_{\mathrm{DP}}$ provide are indicated on the top, where $\mathrm{H}$ and $\mathrm{P}$ denote hydrophobic and polar interactions, respectively. 


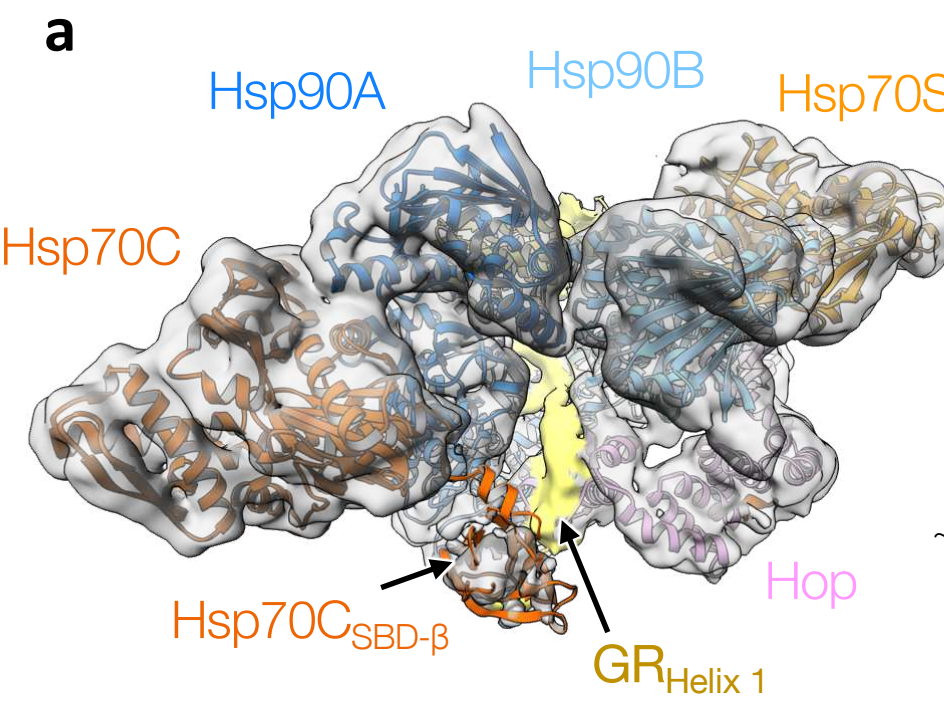

\section{b}
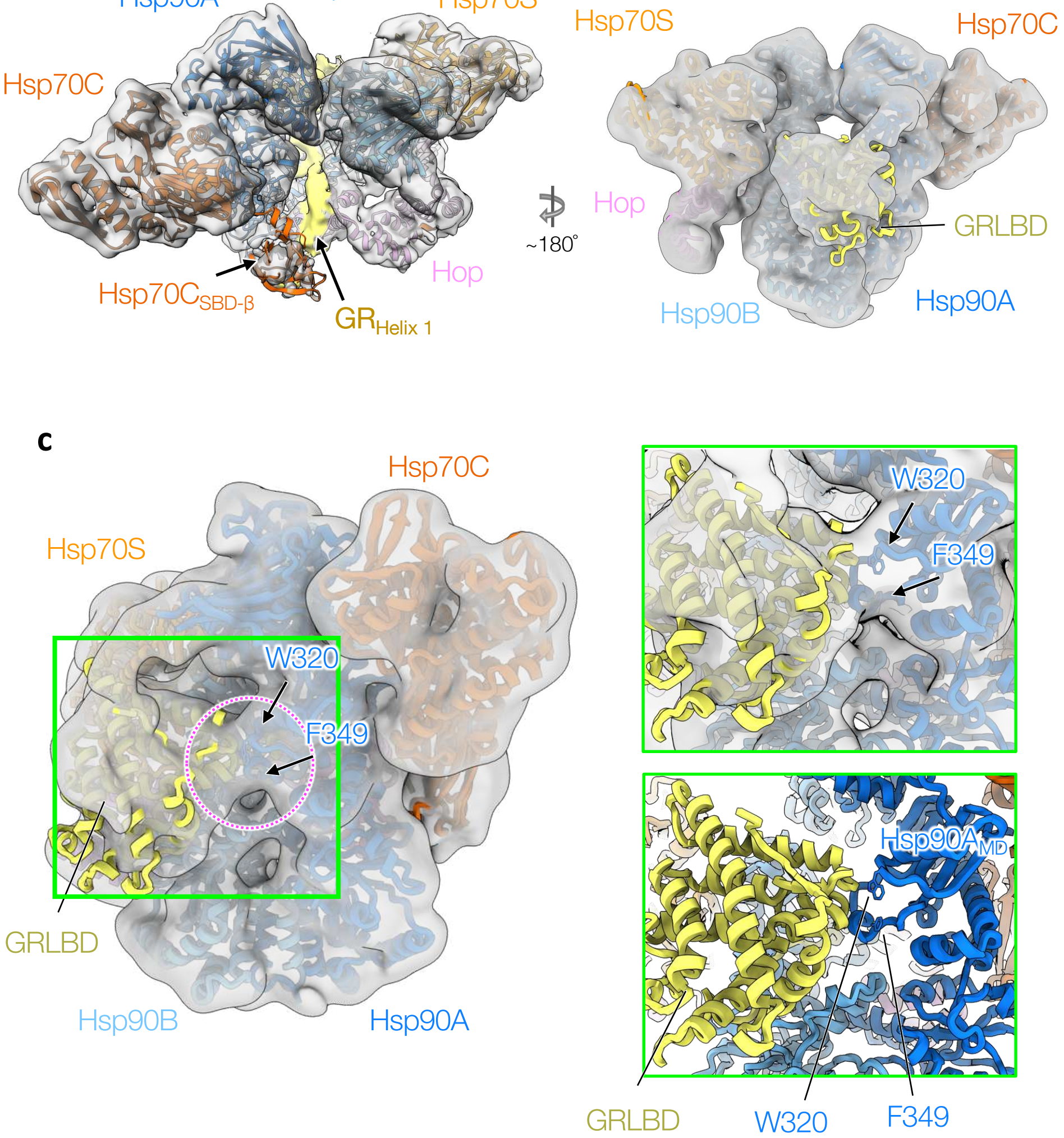

Extended Data Fig. 24 | GR is unfolded, threaded through the Hsp90 lumen and bound by Hsp90, Hop and Hsp70 density. a, A lumen density of GR connects to the globular part of GR on the other side of Hsp90. b, Docking of the GRLBD to the low-pass filtered map shows that the lowresolution GR density can fit the rest of the GRLBD. c, The low-pass filtered map shows that W320 and F349 (arrows) of Hsp90A in the loading complex are in contact with GRLBD. 


$\begin{gathered}\text { Linker to } \\ \text { MBP }\end{gathered}$ GR $_{\text {pre-Helix } 1}$ Helix $1 \quad$ GRLBD
FQSPGS IVPATLPQLTPTLVSLLEVIEPEVLYAGYDSSVP

Negative deuterium uptake (HDX-MS)

Hsp70 binding site
predictions $\left[\begin{array}{r}\text { BiP Pred } \\ \text { ChaperISM } \\ \text { ChaperISM } \\ \text { BiP Pred } \\ \text { ChaperISM }\end{array}\right.$

b

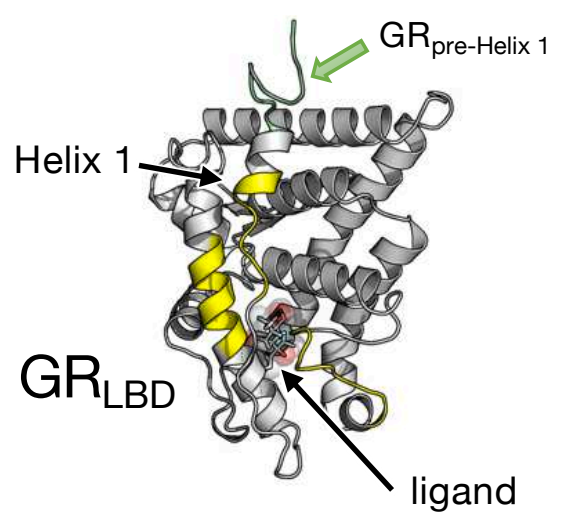

\section{PGSIVPATLPQLTPTL}

\begin{tabular}{c} 
S IVPATL \\
\hline SIVPATL \\
IVPATLP \\
TIVPATL \\
TIVPATL
\end{tabular}

$\Delta$ native GR sequence

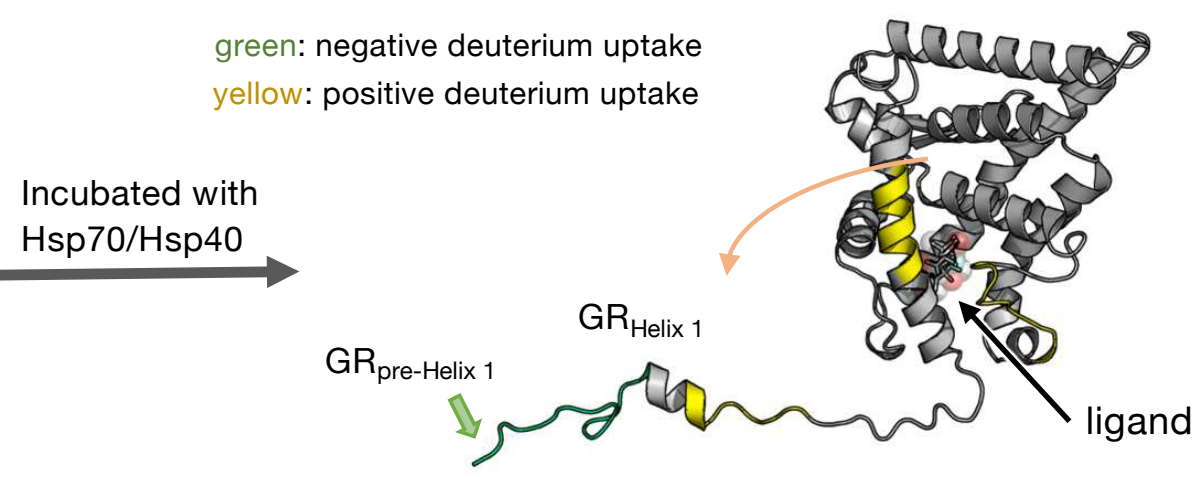

C
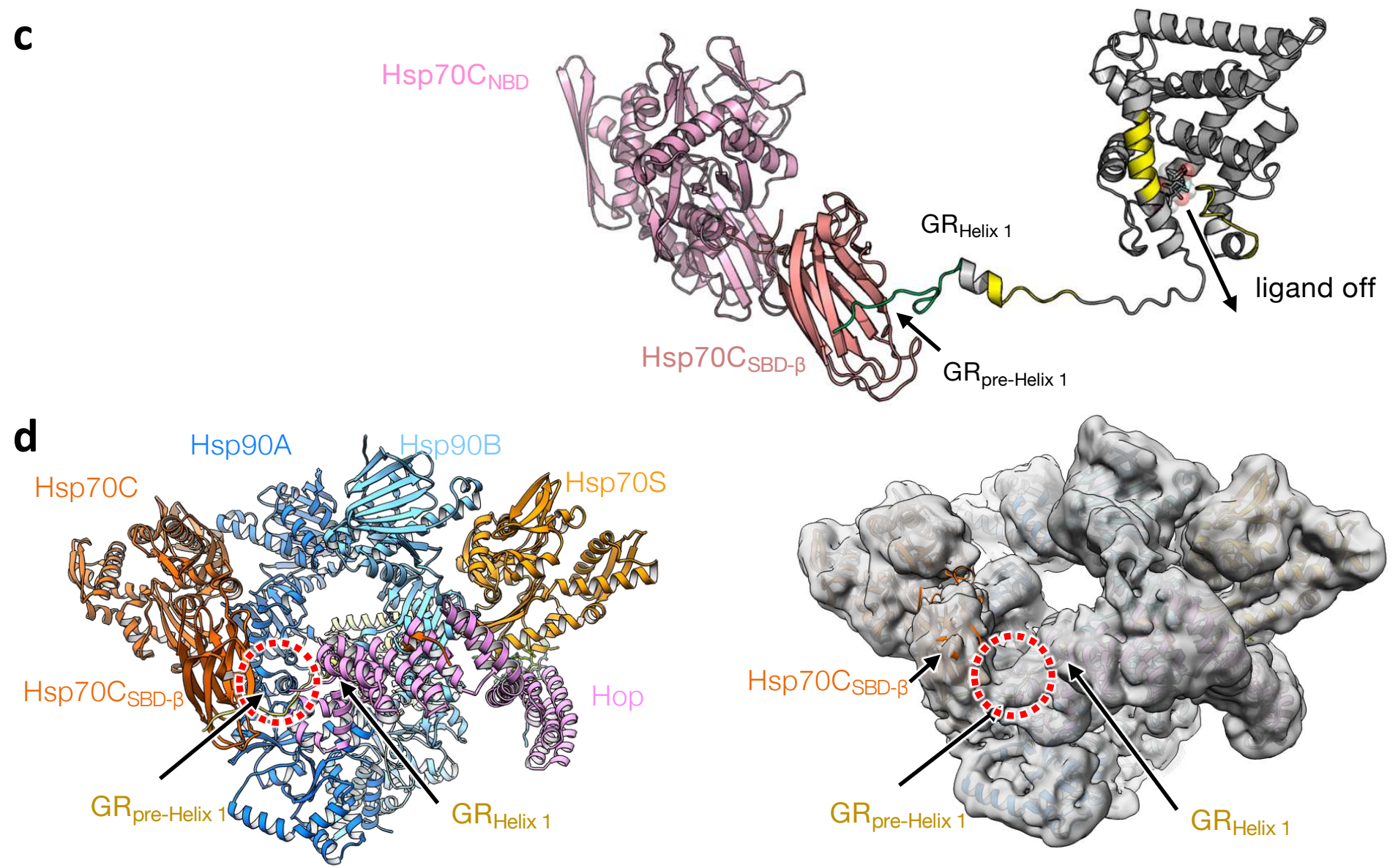

Extended Data Fig. 25 Hsp70 inhibits GR by binding the pre-Helix 1 region of GR. a, After engaging with Hsp70/Hsp40, the GR exhibits negative deuterium uptake in a HD-exchange mass spectrometry (HDX-MS) experiment (Kirschke et al., 2014). In the GR there are Hsp70 binding sites predicted by two state-of-the-art algorithms (BiP Pred and ChaperlSM). b, Left, GRLBD crystal structure (1m2z) colored by the change of deuterium uptake (HDX-MS was retrieved from Kirschke et al., 2014); green: negative; yellow: positive. Right, the detachment of the entire GR Helix 1 motif explains the positive deuterium uptake around the ligand-binding pocket. c, The negative uptake of the pre-Helix region can be explained by binding of Hsp70. Together (b) and (c) provide a molecular mechanism describing how Hsp70 inhibits GR igand binding. d, GR's pre-Helix 1 remains bound to Hsp70 (red circles) in loading complex. Left, atomic model with ribbon presentation. Right, a low-pass filtered cryo-EM map of the loading complex. 
a
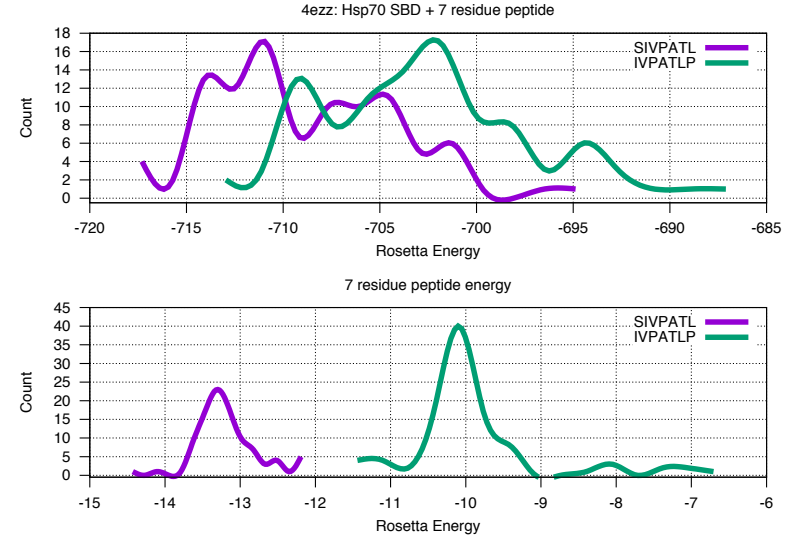

C
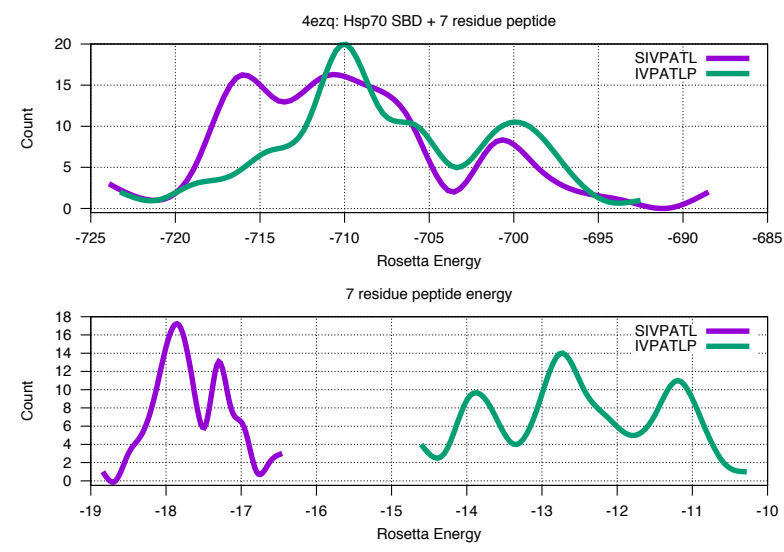

b
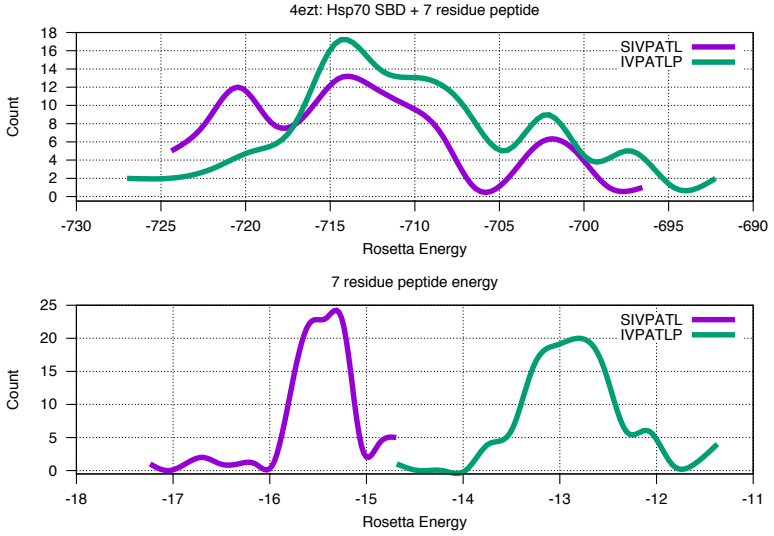

d

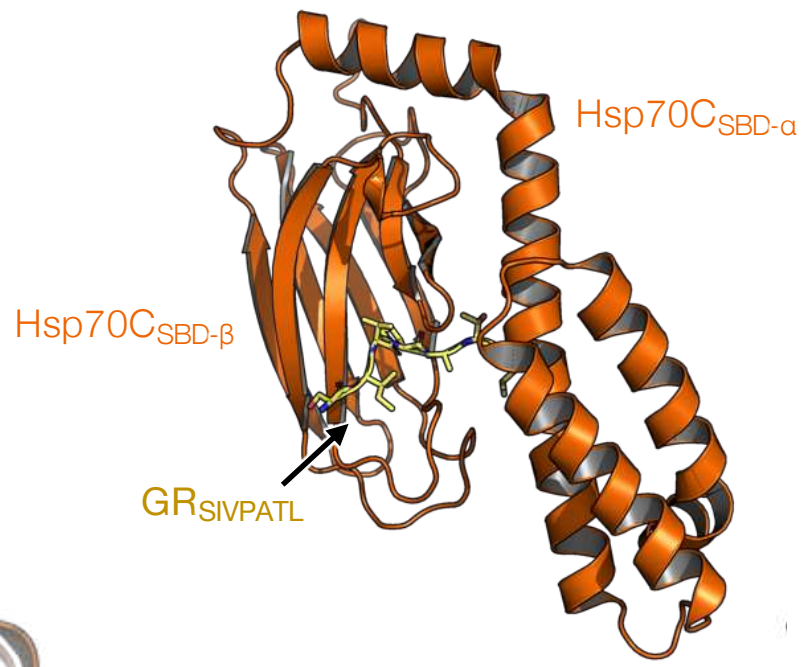

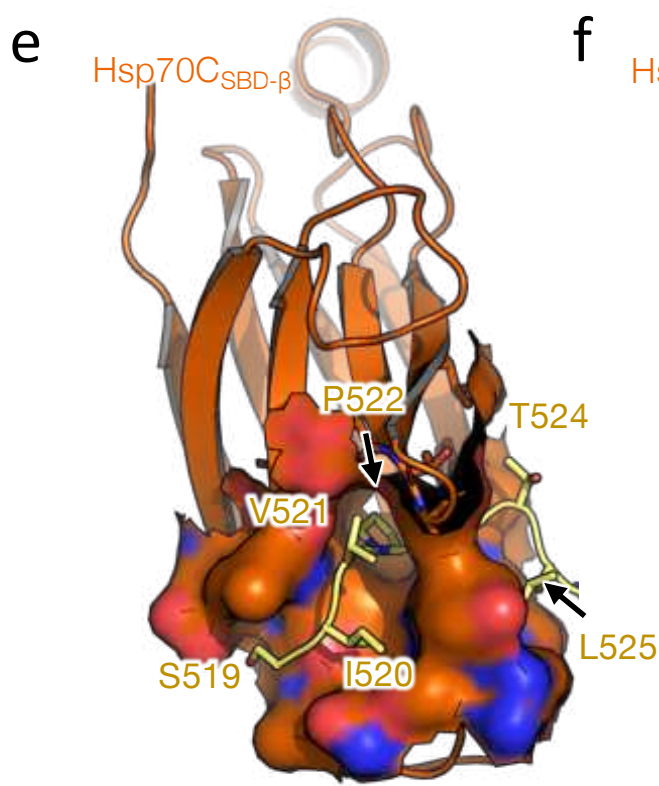
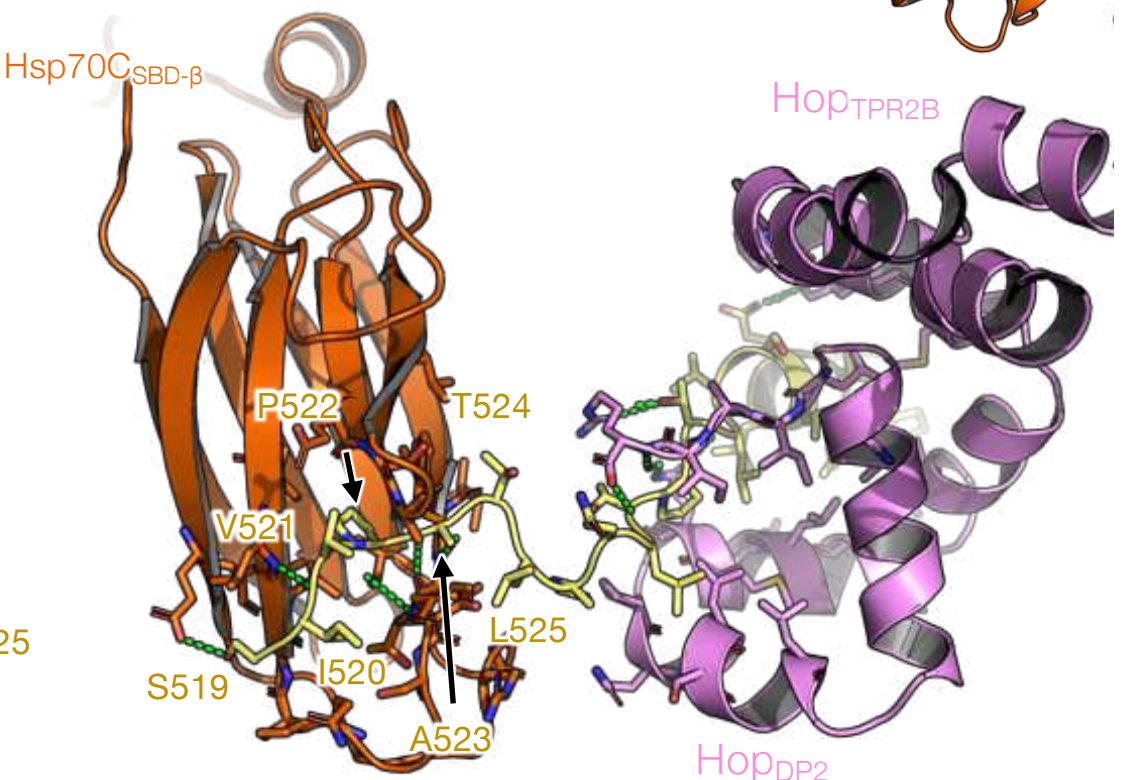

Extended Data Fig. 26 | Determination of the GR segment that Hsp70C SBD $_{\text {B }}$ binds. a, Two 7-residue GR segments (SIVPATL and IVPATLP) of a continuous sequence (residues 518-525; note that residue 518 in the native GR sequence is T, not S) in the pre-Helix 1 regions are predicted as Hsp70 binding sites. Using the crystal structures of E. coli Hsp70 $\mathrm{sBD}$ (DnaK) with "reverse" binding peptides as templates (PDB ID: 4ezz (a), 4ezt (b), and 4ezq (c)), sequences of the two GR segment and human Hsp70 were threaded to the backbone of the crystal structures, resulting in two starting homology models of $\mathrm{Hsp} 70_{\mathrm{SBD}}$ :peptide for each template. Rosetta full-atom energy minimization was carried out for the individual threaded models. 100 models were generated for each threaded model. Density plots show the energy distribution (Y-axis=count) of the 100 models, where the top plot shows the total energy ( $X$-axis) from Hsp70:peptide and the bottom plot shows only energy contributed by the peptide. b, Same as (a), but using 4ezt as the template. c, Same as (b), but using 4ezq as the template. d, The selected model, which was the lowest energy model from the job using $4 \mathrm{ezq}$ as the template and SIVPATL as the bound peptide. e, Closeup view of the Hsp70 $\mathrm{SBD}$ from (d) with surface representation with positive/negative charged residues colored in blue/red (left) and stick representation (right) around the peptide binding pocket. f, Atomic model of Hsp70 loading GR to Hop DP2. Green dashed lines indicate polar interactions. 


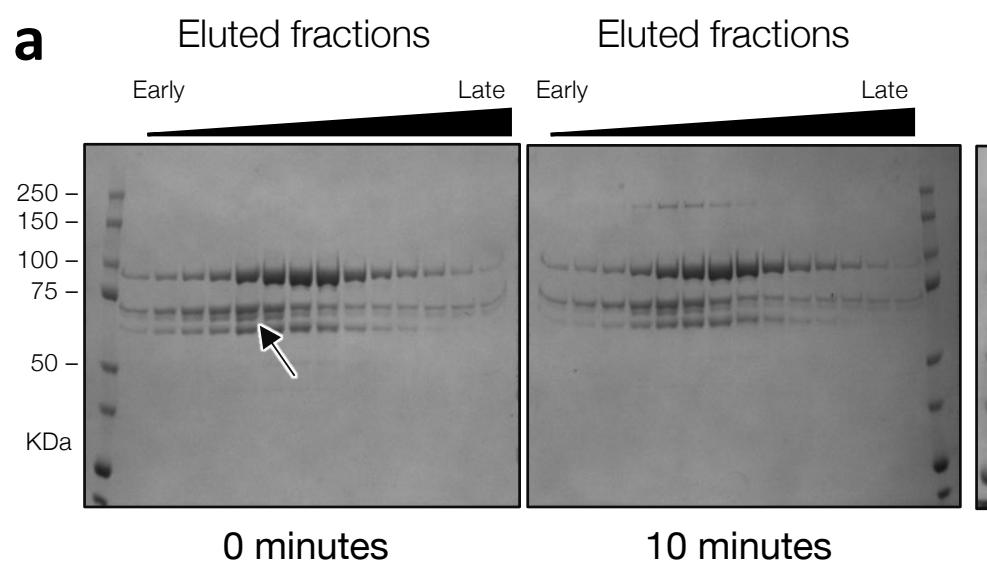

b

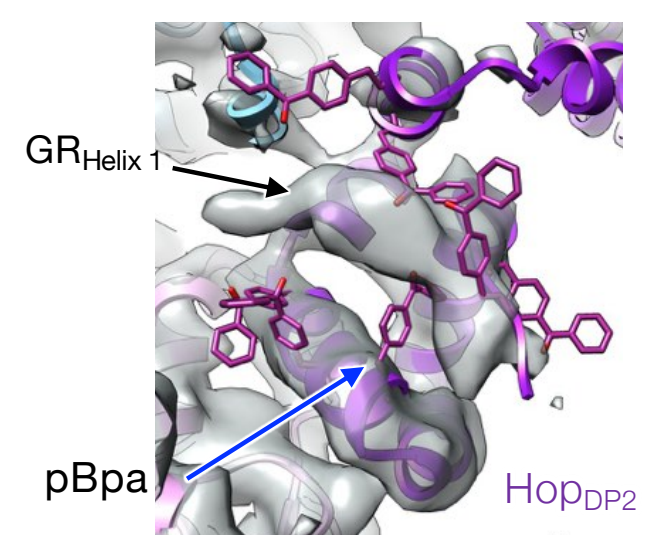

Eluted fractions

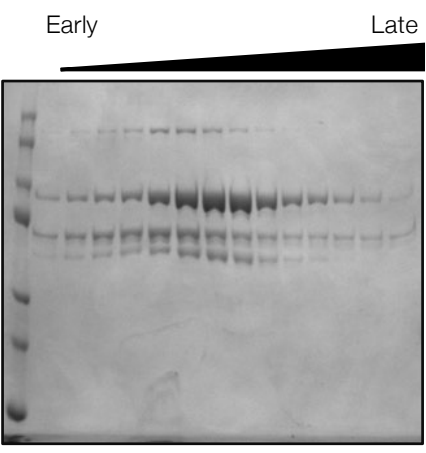

30 minutes
Eluted fractions

Late Early Late

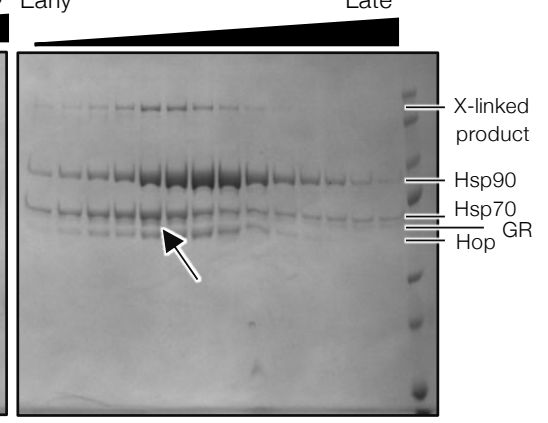

60 minutes

Western blot

C
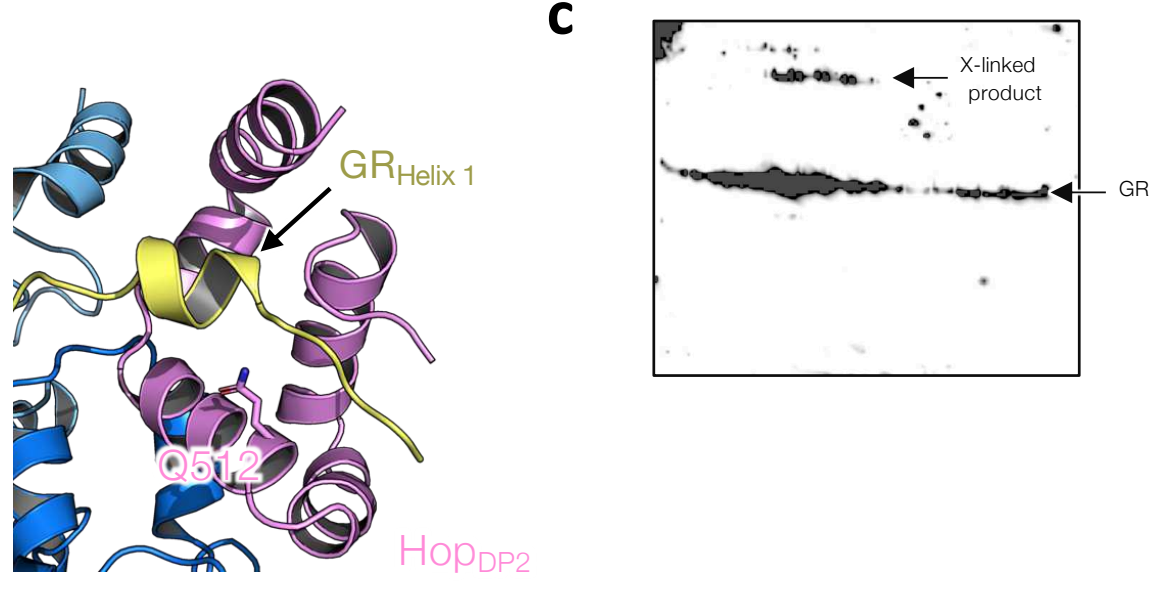

Extended Data Fig. 27 | The density Hop ${ }_{D P 2}$ binds belongs to GR. a, A time course of UVexposed GR-loading complex analyzed by SDS-PAGE and visualized by Coomassie staining. Whole fractions of GR-loading complex eluted from the size-exclusion column were exposed to UV using a gel imager. Arrows at 0 and 60 minutes indicate a reduced intensity of the GR band over the time course. $\mathbf{b}$, Modeling the photoreactive crosslinker p-benzoyl-L-phenylalanine (pBpa) (magenta) on various positions of HopDP2. The blue arrow on the left panel points at the selected position, Q512 (right panel). c, Western blot of the SDS-PAGE gel after a 60-minute UV exposure, using anti-MBP antibody to detect the MBP-tagged GR. 
a
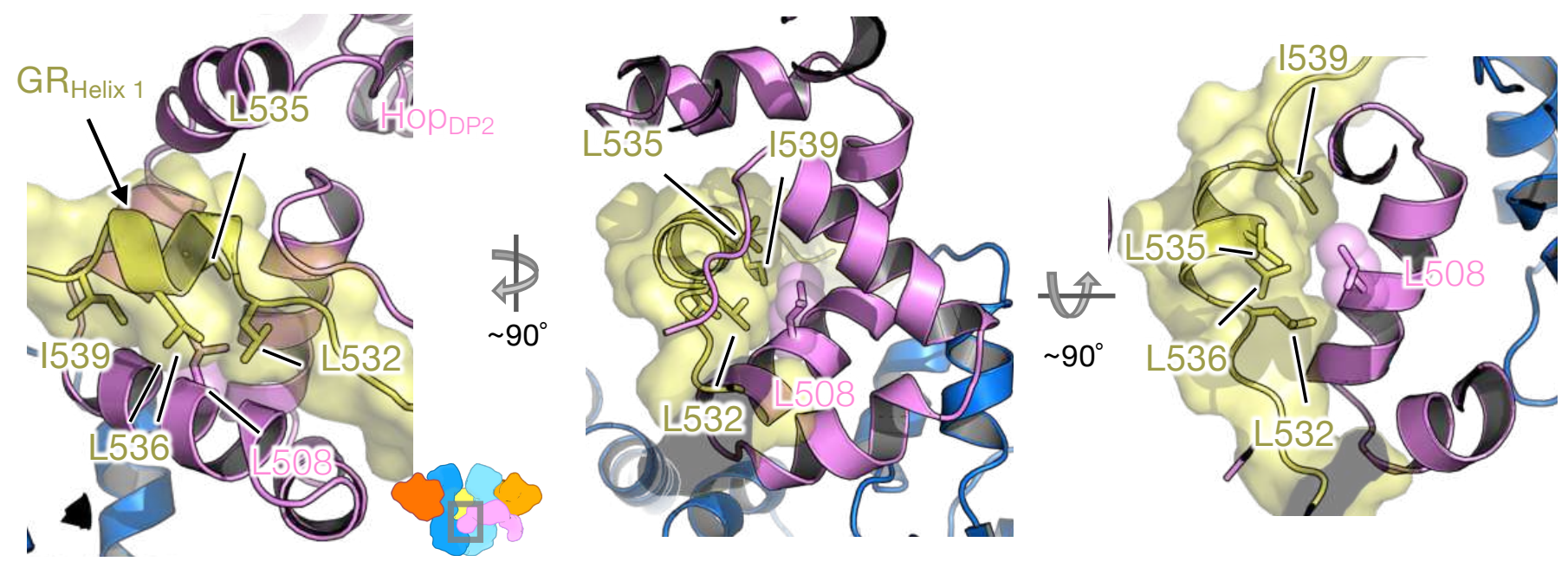

Extended Data Fig. 28 | Molecular basis for the effect of the HOP L508A mutation, which completely abrogates GR function in vivo. a, L508 is located on the hydrophobic palm of DP2, interacting closely with the LXXLL motif of $\mathrm{GR}_{\text {Helix } 1}$ through hydrophobic interactions (left, middle and right). 
a

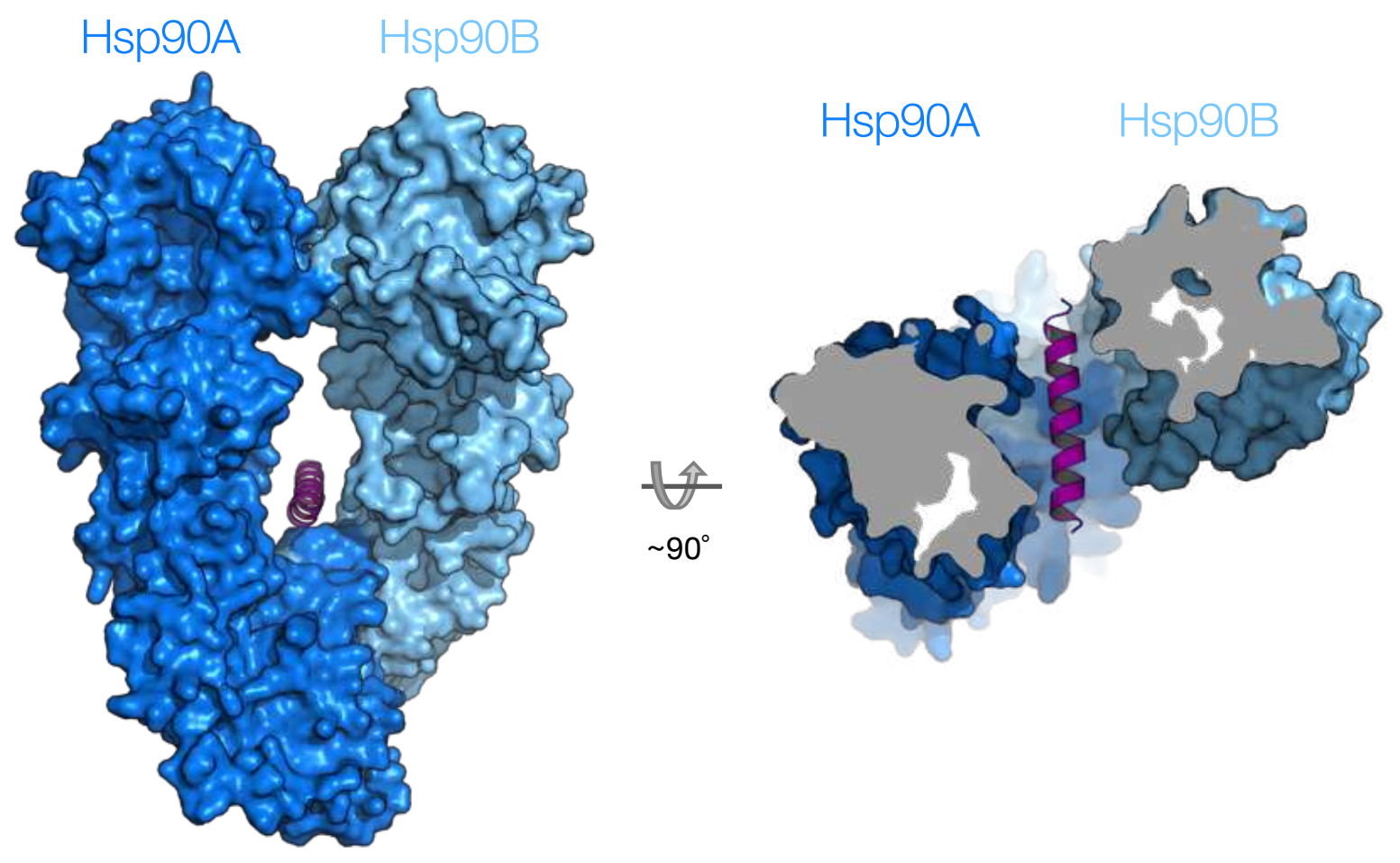

Extended Data Fig. 29 | The lumen of the semi-closed Hsp90 presented in the loading complex can fit a helix. a, A helix (magenta) can be accommodated in the semi-closed Hsp90. Front view (left) and top view (right). 
Extended Data Table 1. Cryo-EM data collection

\begin{tabular}{|c|c|c|c|c|}
\hline Data set & D93N-I & D93N-II & D93N-III & D93N-IV \\
\hline Microscope & Titan Krios & Titan Krios & Titan Krios & Titan Krios \\
\hline Camera & K2 Summit & K2 Summit & K2 Summit & K2 Summit \\
\hline \multicolumn{5}{|c|}{ Data collection and processing } \\
\hline Magnification & 22,500 & 22,500 & 22,500 & 22,500 \\
\hline Voltage (kV) & 300 & 300 & 300 & 300 \\
\hline Electron Exposure (e-/Å2) & 80 & 80 & 70 & 70 \\
\hline Exposure time (sec) & 12 & 16 & 14 & 14 \\
\hline Defocus range $(\mu \mathrm{m})$ & -0.5 to -2.3 & -0.5 to -2.5 & -1.0 to -2.5 & -0.5 to -2.5 \\
\hline Pixel Size (Å/pixel) & 1.059 & 1.059 & 1.059 & 1.059 \\
\hline Micrographs collected (no.) & 3145 & 3213 & 489 & 4248 \\
\hline Particles picks (no.) & 986,519 & \multicolumn{2}{|c|}{$1,397,986^{\star}$} & $1,728,005$ \\
\hline Refined particles (no.) & 139,412 & \multicolumn{2}{|c|}{$214,315^{\star}$} & 282,329 \\
\hline
\end{tabular}

* Data sets II and III were merged and processed together. 


\begin{tabular}{|c|c|c|c|c|c|c|}
\hline Map & Hsp90:Hsp70:Hop:GR & $\begin{array}{l}\text { Hsp90A }_{\text {NTD-MD: }} \\
\text { Hsp70C }\end{array}$ & $\begin{array}{l}\text { Hsp90B }_{\text {NTD-MD: }} \\
\text { Hsp70S }_{\text {NBD }}\end{array}$ & $\begin{array}{c}\text { Hsp90B }{ }_{\text {NTD-MD }}: \\
\text { Hsp70S }_{\text {NBD }}: H_{\text {TPR2A-TPR2B-DP2 }}\end{array}$ & $\mathrm{Hsp} 70 \mathrm{~S}_{\mathrm{NBD}}: \mathrm{Hop}_{\mathrm{TPR} 2 \mathrm{~A}}$ & $\begin{array}{c}\text { Hsp90AB } \text { CTD }_{\text {Tspp70S }}: \mathrm{S}_{\mathrm{SBD}-\beta}: \\
\text { Hop }_{\mathrm{DP} 2}: \mathrm{GR}_{\text {Helix } 1}\end{array}$ \\
\hline EMDB ID & EMD-23050 & EMD-23053 & EMD-23054 & EMD-23056 & EMD-23055 & EMD-23051 \\
\hline PDB ID & 7KW7 & & & & & \\
\hline Classification type & Global & $\begin{array}{c}\text { Focused } \\
\text { (signal subtraction) }\end{array}$ & $\begin{array}{c}\text { Focused } \\
\text { (signal subtraction) }\end{array}$ & $\begin{array}{c}\text { Focused } \\
\text { (signal subtraction) }\end{array}$ & $\begin{array}{c}\text { Focused } \\
\text { (signal subtraction) }\end{array}$ & $\begin{array}{c}\text { Focused } \\
\text { (signal subtraction) }\end{array}$ \\
\hline $\begin{array}{l}\text { Resolution ( } \AA \text { ) } \\
\text { at FSC } 0.143\end{array}$ & 3.57 & 3.77 & 3.53 & 3.85 & 3.64 & 3.46 \\
\hline Estimated B-factor $\left(\AA^{2}\right)$ & -107.5 & -156.4 & -91.5 & -150.0 & -116.4 & -112.6 \\
\hline Refined particles (no.) & 85619 & 35024 & 56945 & 37845 & 60433 & 43626 \\
\hline \multicolumn{7}{|l|}{ Coordinates refinement } \\
\hline $\begin{array}{l}\text { Initial models used } \\
\text { (PDB code) }\end{array}$ & $\begin{array}{c}\text { Hsp90: 3T0H, 3Q6M, 5FWK } \\
\text { Hsp70: 3AY9, 4PO2, 4EZQ } \\
\text { Hop: 3UQ3, 2LLW } \\
\text { GR: 1M2Z }\end{array}$ & & & & & \\
\hline $\begin{array}{l}\text { Resolution ( } \AA \text { ) } \\
\text { at FSC } 0.5\end{array}$ & 3.99 & & & & & \\
\hline $\begin{array}{l}\text { Map sharpening B-factor for } \\
\text { modeling }\left(\AA^{2}\right)\end{array}$ & -80 & & & & & \\
\hline \multicolumn{7}{|l|}{ Model composition } \\
\hline Non-hydrogen atoms & 21854 & & & & & \\
\hline Protein residues atoms & 21796 & & & & & \\
\hline Ligand atoms & 58 & & & & & \\
\hline \multicolumn{7}{|l|}{ Mean B-factor (min - max) [ $\left.\AA^{2}\right]$} \\
\hline Protein residues & $202.8(18.8-600.0)$ & & & & & \\
\hline Ligands & $118.4(62.8-202.0)$ & & & & & \\
\hline \multicolumn{7}{|l|}{ Model geometry (r.m.s.d.) } \\
\hline Bond lengths $(\AA)$ & 0.0209 & & & & & \\
\hline Bond angles $\left({ }^{\circ}\right)$ & 1.73 & & & & & \\
\hline \multicolumn{7}{|l|}{ Ramachandran plot } \\
\hline Favored (\%) & 98.71 & & & & & \\
\hline Allowed (\%) & 1.25 & & & & & \\
\hline Disallowed (\%) & 0.04 & & & & & \\
\hline \multicolumn{7}{|l|}{ Validation } \\
\hline MolProbity score & 0.76 & & & & & \\
\hline Clash score & 0.82 & & & & & \\
\hline Poor rotamers $(\%)$ & 0.00 & & & & & \\
\hline
\end{tabular}




\section{Figures}

a

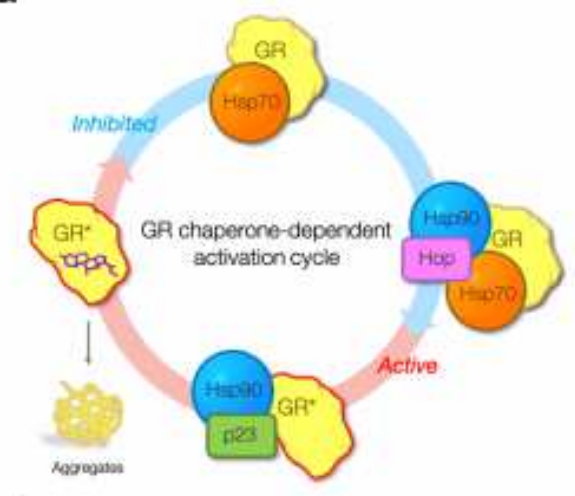

d

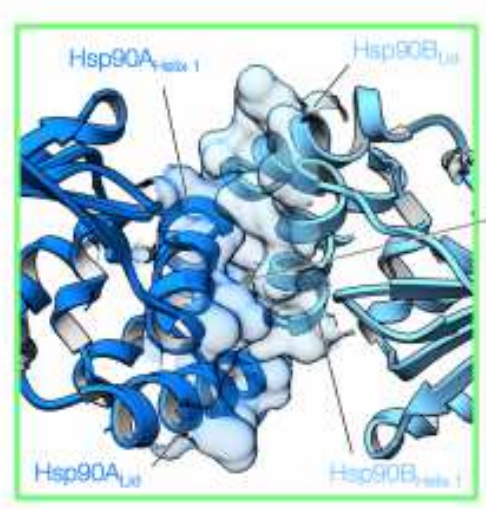

b

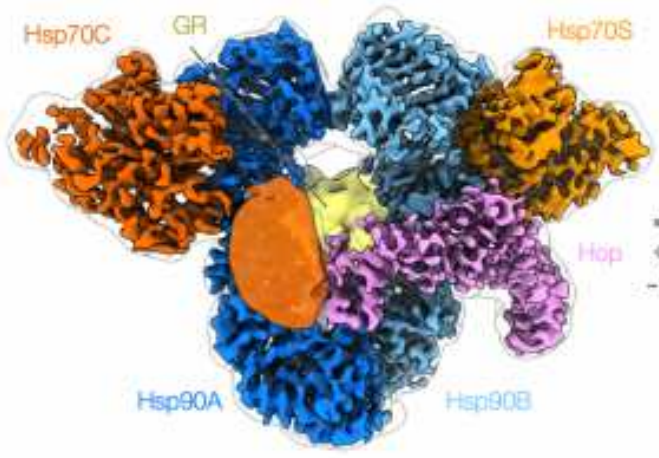

e

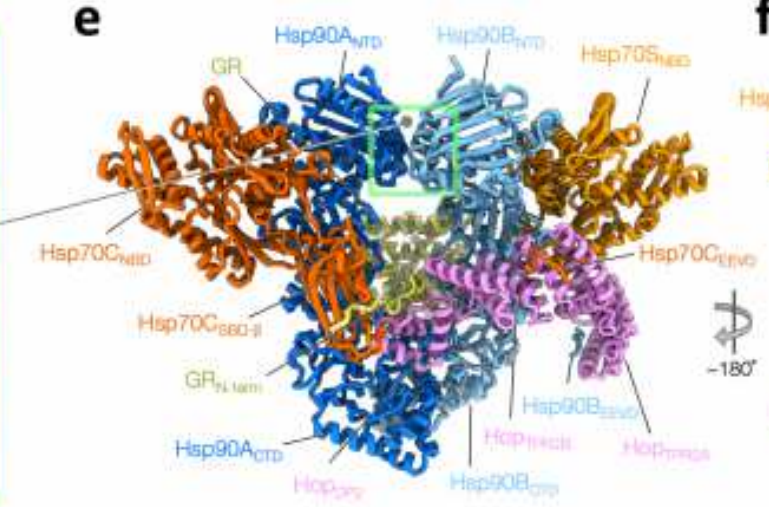

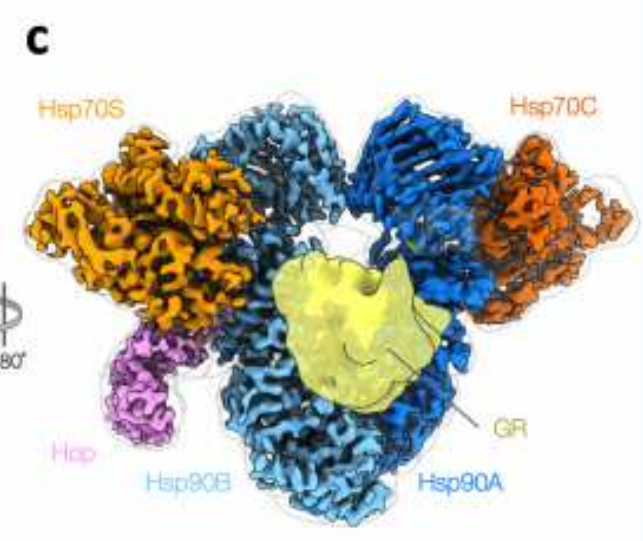

f

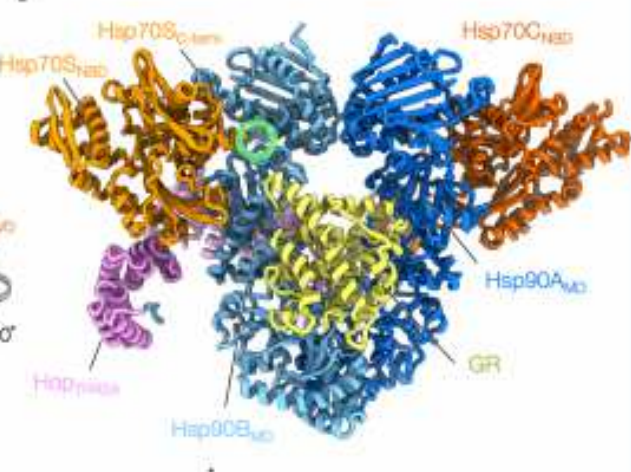

Figure 1

Overview of the GR-loading complex. a, GR activity is regulated by molecular chaperones in a constant cycle. Starting from active GR (left), which is aggregation-prone (lower left) in physiological conditions, Hsp70 protects and inhibits GR (top). Recruited by Hop, Hsp70 loads GR to Hsp90, forming the loading complex, in which GR remains inactive. Upon ATP hydrolysis on Hsp90, GR is reactivated in the maturation complex of GR:Hsp90:p23 (bottom) and is thereafter released to continue the cycle. b,c, Front (b) and back (c) views of a composite cryo-EM map of the GR:Hsp90:Hsp70:Hop complex. Densities of Hsp70CSBD and the globular C-terminal GR (yellow) are taken from the low-pass-filtered map of the highresolution reconstruction of the full complex. The subunit color code is used throughout. d, Close-up view of the novel dimerization interface of the symmetric Hsp90 dimer with residues at the interface in surface representation. The interface is composed of two molecular switches of Hsp90, the first helix and the lid motif. e,f, Corresponding views of the atomic model of the GR-loading complex. Green circle indicates the C-terminus of Hsp70S. 


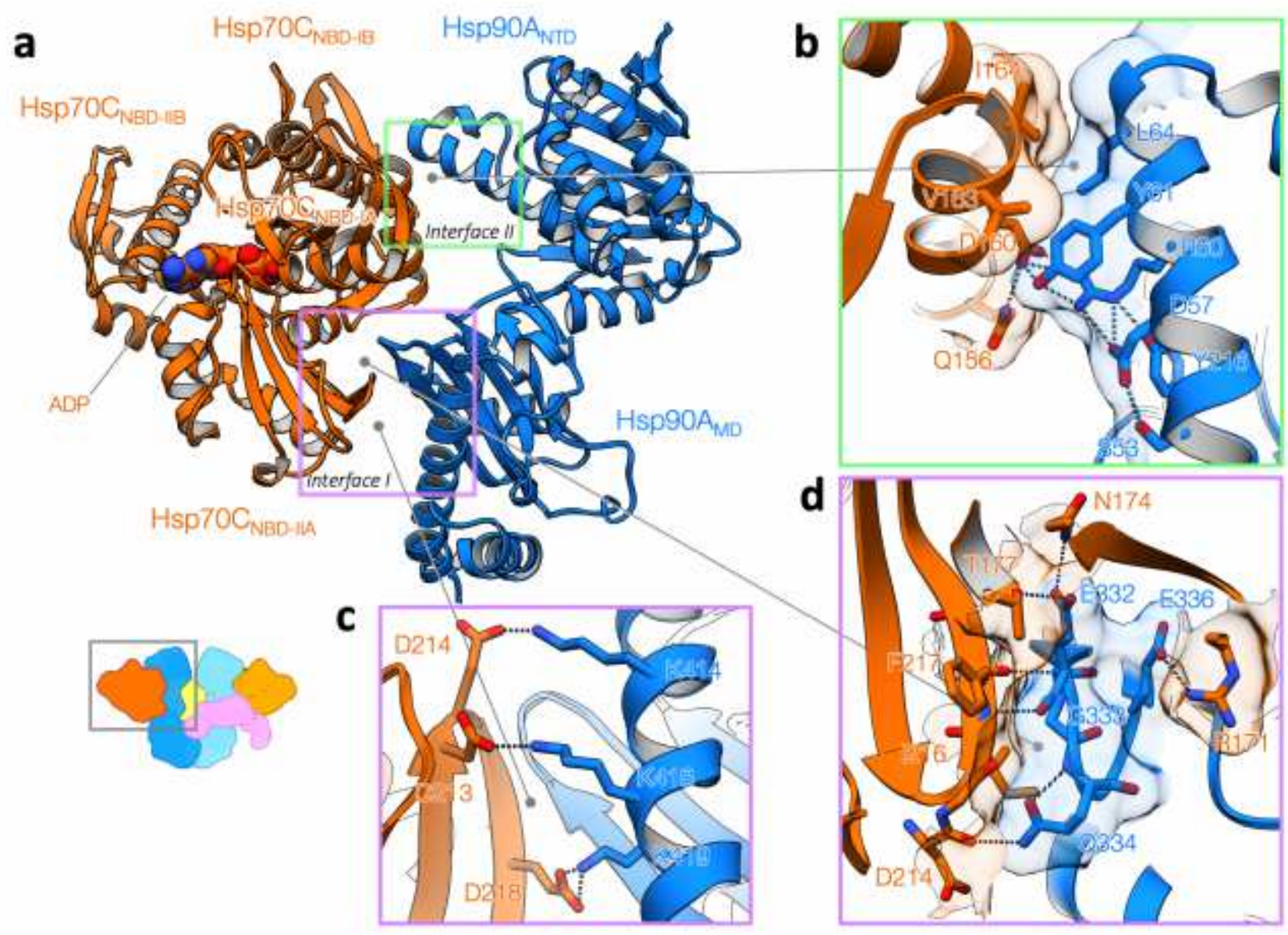

Figure 2

Molecular basis of Hsp90:Hsp70 interactions. a, Hsp90:Hsp70 interactions without the aid of Hop (Hsp90A:Hsp70C). In Interface I (purple rectangle), Hsp90 uses the outer edge of the Hsp90AMD $\beta$-sheet to insert into a cleft formed by Hsp70NBD-IA and Hsp70NBD-IIA subdomains. The cleft is where the Hsp70 interdomain linker binds and serves as the allosteric center for Hsp70 NBD to regulate client binding in the Hsp70 SBD. In Interface II (green rectangle), the two ATPase domains Hsp90 and Hsp70 directly interact with each other. b, Close-up view of Interface II. Transparent surface and stick representations are shown for residues involved in the interactions. Dashed lines depict the network of polar interactions involved. $c$, Interface I is featured with conserved salt bridges (dashed lines). d, Detailed view of Interface II. A $\beta$-strand pairing (dashed lines) between the backbone atoms of Hsp90E332 and Hsp70F217, in which Hsp90G333 is closely packed with Hsp70F217. 


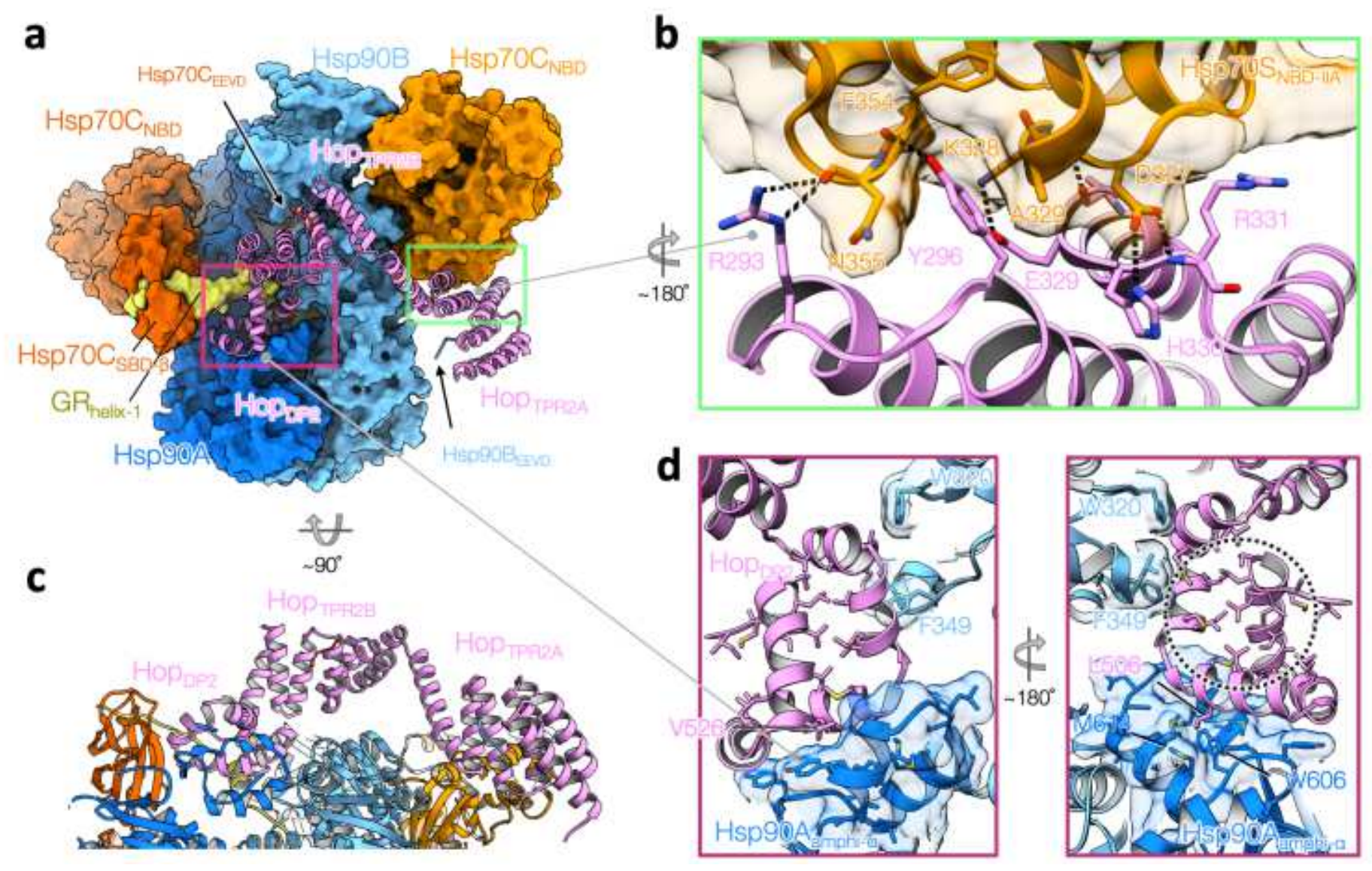

\section{Figure 3}

Hop interacts intimately with all components in the loading complex. a, Hop, shown in ribbon representation, uses its three C-terminal domains (HopTPR2A, HopTPR2B and HopDP2) to interact with Hsp90/Hsp70 beyond the EEVD binding. All the other components are shown in surface representation. The red rectangle highlights that HopDP2 interacts with Hsp90A (dark blue), Hsp90B (light blue), Hsp70CSBD (dark orange) and a portion of GR (yellow). The green rectangle highlights a novel interface formed by HopTPR2A and Hsp70CNBD-IIA. b, Close-up view of the novel Hop:Hsp70 interface with a 180degree rotation from (a). HopY296 inserts into a cavity on Hsp70 (transparent surface representation), forming a hydrogen bond with the Hsp70F354 backbone atom. The interface also features many polar interactions depicted with dashed lines. c, A 90-degree rotation from the bottom view in (a) with ribbon model. No major interaction is observed between HopTPR2B and the loading complex. d, Left, HopDP2 uses surfaceexposed hydrophobic residues, shown in sticks, to interact with Hsp90's client-binding motifs (shown in transparent surface and with hydrophobic residues in sticks)-- the amphipathic helical hairpin from Hsp90ACTD (dark blue) and Hsp90BW320,F349 (light blue). Right, a 180-degree view from the left, HopDP2 adopts a hand-like a-helical structure. The core of HopDP2 is loosely packed with many hydrophobic residues exposed in the "palm" (black circle) of the "hand". Note the GR-binding to HopDP2 is not shown here. 

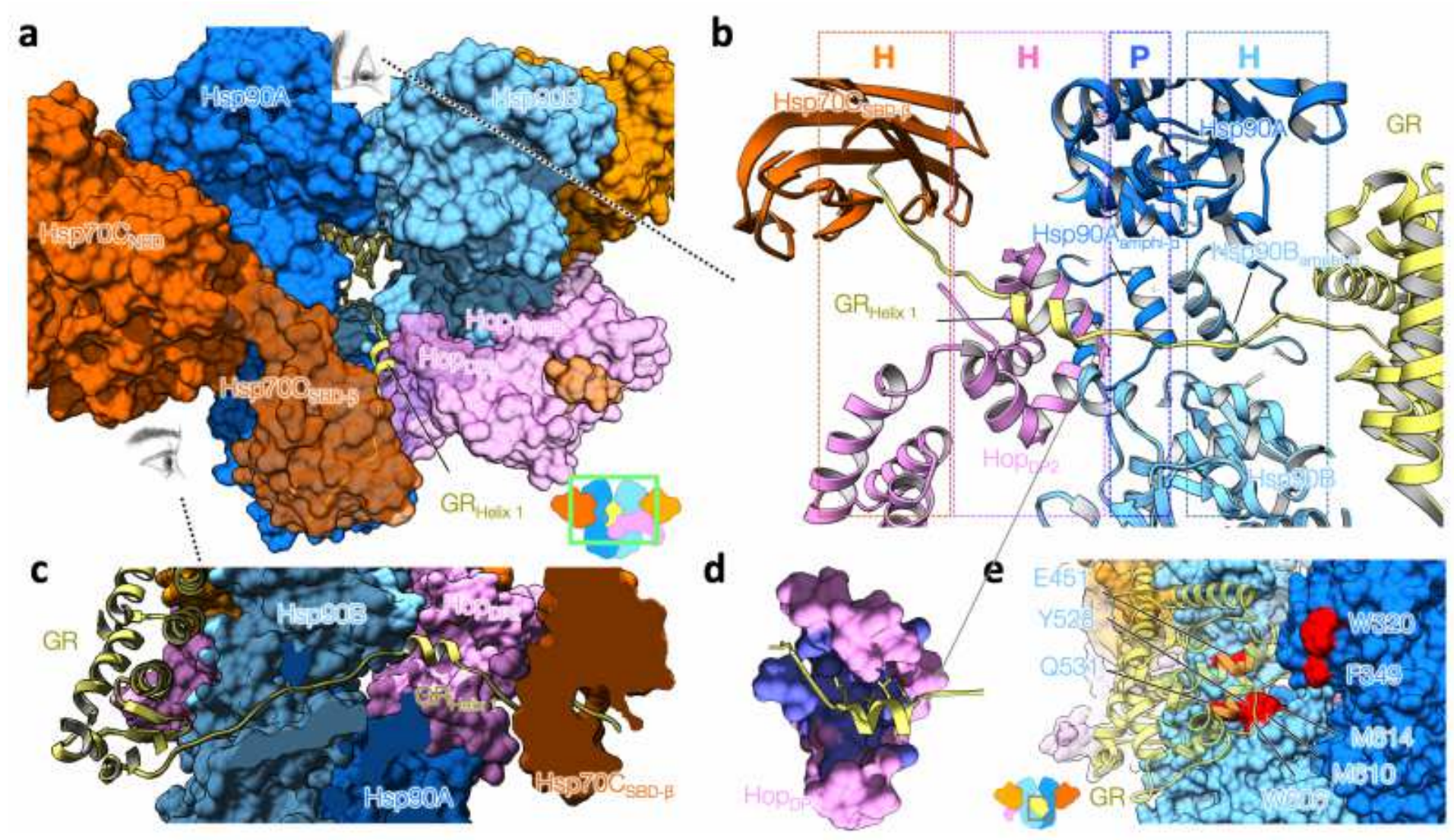

Figure 4

Facilitated by Hop, GR is loaded onto Hsp90 by Hsp70. a, Close-up front view of the loading complex (shown in surface representation). GR, shown in the ribbon model, is partially unfolded, with the Nterminal residues simultaneously griped by Hsp70C and HopDP2, and is threaded through the semiclosed lumen of Hsp90. The remaining GR is at the other side of the loading complex; the ribbon model shown for the major body of GR is a modeling result from docking the GR crystal structure to the lowpass-filtered GR density. b, Top- to-bottom view of GR recognition via an extended client-binding pocket collectively formed by Hsp70CSBD- $\beta$, HopDP2, and Hsp90A/Bamphi-as in ribbon representation. The Nterminal residues of GR (residues 517-533), which form a strand-helix-strand motif (yellow), are captured in the loading complex. The molecular properties provided by the individual binding pockets are color coded and labelled on the top panel ( $\mathrm{H}$ and $\mathrm{P}$ denote hydrophobic and polar interactions, respectively). $\mathrm{C}$, Side view of the GR N-terminal motif captured by the loading complex. d, HopDP2, shown in surface representation, binds the LXXLL motif of GR Helix1, in which the hydrophobic residues of HopDP2 are colored with purple and those of GR are shown in sticks. e, Residues on Hsp90 (surface representation) previously reported to be important for GR (transparent yellow ribbon) activation are highlighted in red. 
a
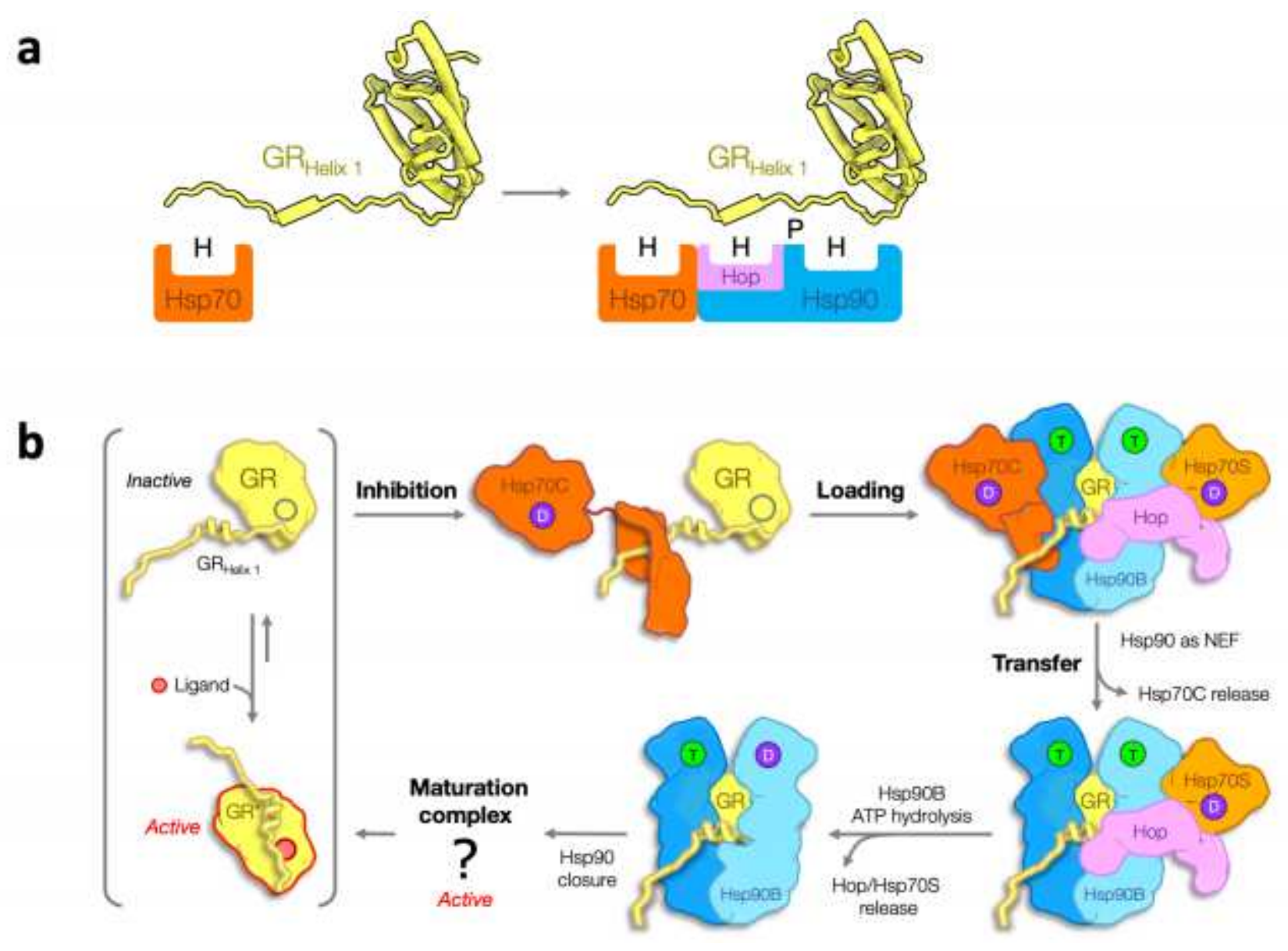

Figure 5

Schematic model of GR loading onto Hsp90 by Hsp70. a, The molecular principle of GR recognition at the client-loading step. Left, Hsp70SBD (orange) inhibits GR (yellow), providing mostly hydrophobic binding $(H)$. Right, the client-loading complex further stabilizes $G R$ via an extended binding pocket assembled by Hsp70SBD (orange), HopDP2 (pink) and the lumen of Hsp90 dimer (blue), where stronger and more versatile molecular recognitions are provided for both hydrophobic $(H)$ and polar $(P)$ interactions. $b$, Molecular mechanism of GR transfer from Hsp70 to Hsp90 and the use of ATP hydrolysis on Hsp90. GR in physiological conditions is in an equilibrium of active and inactive states, in which the Helix 1 motif acts as a lid to stabilize ligand binding when attached. Hsp70C (dark orange) in its ADP state (D) binds GR's pre-Helix 1 strand, facilitating the following motif to detach and hence inhibit GR (top-middle). Facilitated by HopDP2 recognizing the LXXLL motif on GR Helix 1, Hsp70C loads GR to Hsp90 (blue), forming the client-loading complex (top-right). Although the structure was determined in an Hsp90Apo state, we reason that in physiological conditions the high abundance of ATP would soon occupy Hsp90's ATP binding pockets (T). Hsp90's ATP binding and NEF activity facilitate Hsp70C release (bottom-right). The energy from the ATP hydrolysis (D) on Hsp90B (light blue) is used to release Hsp70S (light orange) and Hop (bottom-middle), followed by full closure of Hsp90. 